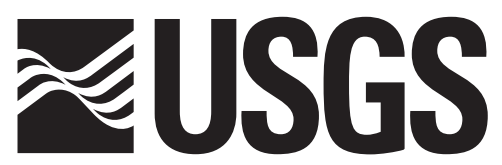

science for a changing world

In cooperation with the City of Houston and the Harris County Flood Control District

\title{
Surface-Water Hydrologic Data for the Houston Metropolitan Area, Texas, Water Years 1990-95
}

Open-File Report 03-070

U.S. Department of the Interior U.S. Geological Survey 
U.S. Department of the Interior

U.S. Geological Survey

\section{Surface-Water Hydrologic Data for the Houston Metropolitan Area, Texas, Water Years 1990-95}

By Debra A. Sneck-Fahrer, Fred Liscum, and Jeffery W. East

U.S. GEOLOGICAL SURVEY

Open-File Report 03-070

In cooperation with the City of Houston and the Harris County Flood Control District 


\section{U.S. DEPARTMENT OF THE INTERIOR}

Gale A. Norton, Secretary

\section{U.S. GEOLOGICAL SURVEY}

Charles G. Groat, Director

Any use of trade, product, or firm names is for descriptive purposes only and does not imply endorsement by the U.S. Government.

For additional information write to

\section{District Chief}

U.S. Geological Survey

8027 Exchange Dr.

Austin, TX 78754-4733

E-mail: dc_tx@usgs.gov

Copies of this report can be purchased from

U.S. Geological Survey

Information Services

Box 25286

Denver, CO 80225-0286

E-mail: infoservices@usgs.gov 


\section{CONTENTS}

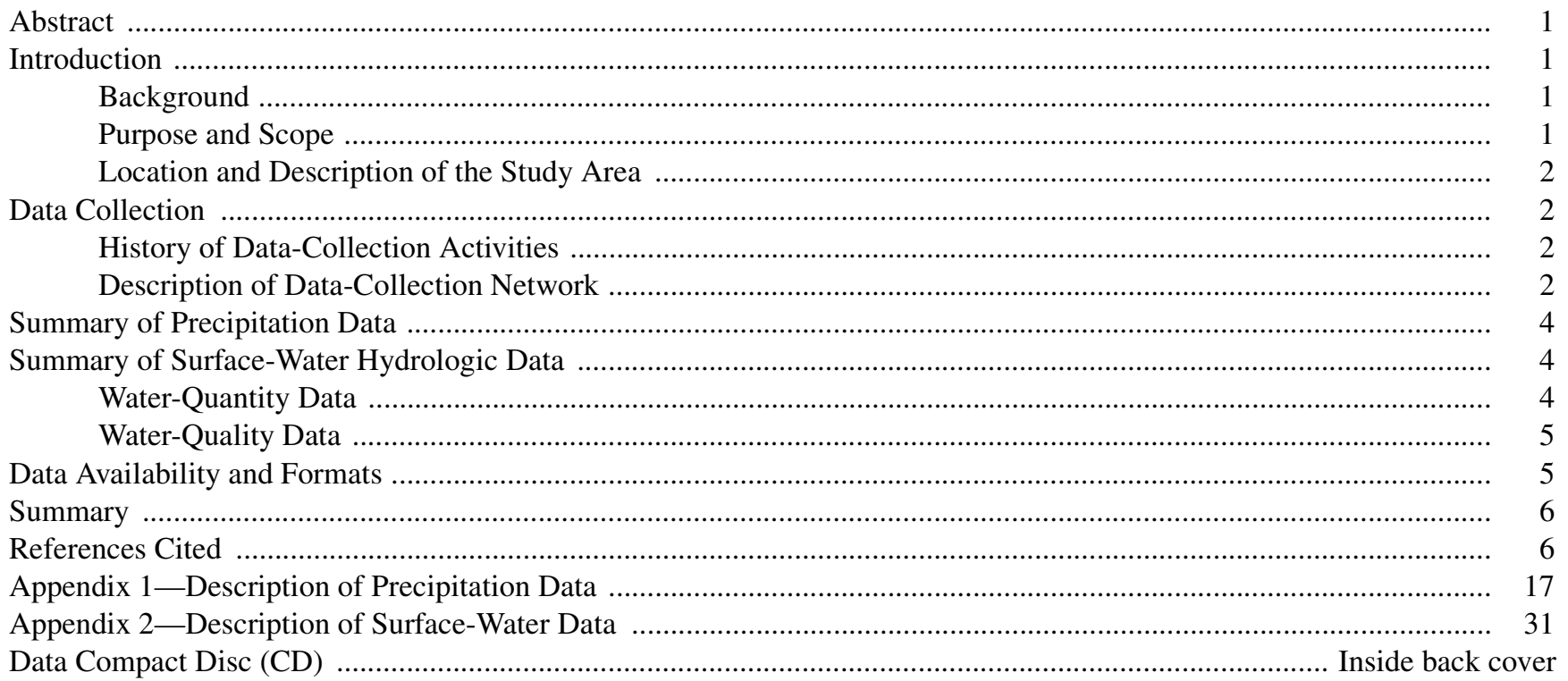

\section{FIGURES}

1. Map showing location of data-collection sites in the Houston metropolitan area, Texas

\section{TABLES}

1. Type of data collected at U.S. Geological Survey stream-gaging stations during water years 1990-95

2. Description of Harris County Office of Emergency Management rain gages used to provide data for this study

3. Observed annual peak discharges for U.S. Geological Survey stream-gaging stations during water years 1990-95

4. Summary of storm-hydrograph data for U.S. Geological Survey stream-gaging stations during water years 1990-95

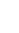
$r$ 


\title{
Surface-Water Hydrologic Data for the Houston Metropolitan Area, Texas, Water Years 1990-95
}

\author{
By Debra A. Sneck-Fahrer, Fred Liscum, and Jeffery W. East
}

\begin{abstract}
During water years 1990-95, data were collected at 24 U.S. Geological Survey streamflowgaging stations, 21 rain gages, and 6 water-quality stations in the Houston metropolitan area, Texas. The data were collected as part of the Houston Urban Runoff Program, which began in water year 1964.
\end{abstract}

Annual peaks were defined for the 24 streamflow-gaging stations in the study area. All stations had 10 or more years of record. Precipitation data from the 21 rain gages and discharge or stage data from 23 streamflow-gaging stations are available to develop storm hydrographs.

One-hundred thirty-four samples were collected at six water-quality stations. The samples were analyzed for about 80 water-quality properties and constituents.

\section{INTRODUCTION}

\section{Background}

Hydrologic investigations of urban watersheds in Texas were begun by the U.S. Geological Survey (USGS) in 1954. Studies have been conducted in most of the major Texas metropolitan areas including Houston, Dallas, San Antonio, and Austin. This report summarizes data collected for the Houston metropolitan area during water years 1990-95 ${ }^{1}$.

The USGS, in cooperation with the City of Houston and the Harris County Flood Control District, began studies of streamflow in the Houston metropolitan area in 1964. The Houston Urban Runoff Program (HURP) was expanded in water year 1968 to include collection of water-quality data. The objectives of the ongoing Houston urban-hydrology study are

\footnotetext{
${ }^{1}$ A water year is the 12 -month period, October 1 through September 30, designated by the calendar year in which it ends.
}

1. To determine, on the basis of historical data and hydrologic analyses of data collected, the magnitude and frequency of flood peaks;

2. To determine the effects of continuing urban development on flood peaks, flood volumes, and characteristics (timing and duration) of flood runoff; and

3. To determine variations in stream water quality for different flow conditions, different seasons, and varying patterns of urban development.

Several studies have used the extensive database compiled since 1964. These studies addressed the effects of urbanization and basin characteristics on flood magnitude and frequency (Johnson and Sayre, 1973; Liscum and Massey, 1980; Liscum, 2001) and the concentrations and loads of selected water-quality constituents discharged into Galveston Bay (U.S. Army Corps of Engineers, 1976). A summary of surface-water and precipitation data collected as part of the HURP during 1964-89 is provided by Liscum and others (1997).

The USGS gratefully acknowledges the support of the various agencies who participated in this study. Principal among these are the City of Houston, Harris County Flood Control District, and Harris County Office of Emergency Management. The U.S. Army Corps of Engineers also has provided support for parts of the study.

\section{Purpose and Scope}

This report presents a summary of selected hydrologic data collected in the Houston metropolitan area during the 1990-95 water years. It is an extension of an earlier report (Liscum and others, 1997) that summarized surface-water hydrologic data for 1964-89. As in the previous report, the USGS focus is the collection of surface-water hydrologic data (stage, streamflow, and water samples to determine chemical quality). However, to develop a more comprehensive database, precipitation data collected by the Harris County Office of 
Emergency Management (HCOEM) are included in the report. The complete database of stage, discharge, precipitation, and water-quality data collected during water years 1990-95 is included on a compact disc (CD) inserted in a pocket on the inside back cover of this report.

\section{Location and Description of the Study Area}

The Houston metropolitan area (study area, fig. 1) in southeast Texas is about 45 miles (mi) north of the Gulf of Mexico on an almost level plain. The landsurface altitude increases from about $35 \mathrm{feet}(\mathrm{ft})$ above NGVD of 1929 in the southeastern part of the study area to about $135 \mathrm{ft}$ above NGVD of 1929 in the northwestern part. Soils in the area predominantly are clay, clay loams, and fine sandy loams of low permeability.

The major stream draining the area is Buffalo Bayou (fig. 1), a tributary of the San Jacinto River. Buffalo Bayou is regulated by Barker and Addicks Reservoirs, flood-detention reservoirs in the western part of the area. From these reservoirs, Buffalo Bayou meanders eastward, is fed by four major tributaries (Whiteoak, Brays, Sims, and Greens Bayous), and enters the Houston Ship Channel and then Galveston Bay on the Gulf of Mexico. The drainage area of Buffalo Bayou, excluding the area above the flooddetention reservoirs, is about 810 square miles $\left(\mathrm{mi}^{2}\right)$.

The Houston metropolitan area has a subtropical humid climate characterized by the predominant onshore flow of tropical maritime air from the Gulf of Mexico. This onshore flow of air is modified by a decrease in moisture from east to west and by intermittent seasonal intrusions of continental air. The climate also is characterized by short and mild winters and hot summers. On the basis of 1971-2000 normals, the average maximum daytime temperature in summer is 92.3 degrees Fahrenheit $\left({ }^{\circ} \mathrm{F}\right)$ (33.5 degrees Celsius $\left.\left[{ }^{\circ} \mathrm{C}\right]\right)$, and the average daily temperature in winter is between 40 and $65^{\circ} \mathrm{F}\left(4\right.$ and $18^{\circ} \mathrm{C}$ ) (Office of the Texas State Climatologist, 2002).

The Houston metropolitan area has developed rapidly. The population grew from 1.24 million in 1960 to 3.32 million in 1990 (U.S. Census Bureau, 2002). From 1990 to 1995, the population increased 10.3 percent to 3.70 million people (Real Estate Center at Texas A\&M University, 2002).

\section{DATA COLLECTION}

\section{History of Data-Collection Activities}

From the beginning of the HURP program in 1964, the USGS has installed and maintained streamflow-gaging instruments to collect the required data on most major drainage basins in the metropolitan area (Buffalo, Whiteoak, Brays, Sims, and Greens Bayous) and many minor basins (including Keegans, Little Whiteoak, Berry, Vince, Hunting, and Halls Bayous). By 1966, the HURP data network consisted of 27 rain gages and 33 streamflow-gaging stations (19 continuous; 14 flood hydrograph) maintained by the USGS. The maximum number of instrumented streamflow stations (39) were in place during water years 1971, 1972, and 1982. Water-quality sampling began with 1 station in water year 1968, increasing to 13 stations in water year 1969. The water-quality network had a maximum of 15 stations during water year 1972. During water years 1990-95, 24 streamflow stations, 21 rain gages (all rain gages operated by the HCOEM), and 6 water-quality stations were monitored (fig. 1). Table 1 (at end of report) lists the type of data collected at each USGS gaging station in the data network during this period.

\section{Description of Data-Collection Network}

The USGS data-collection network consists of several types of gaging stations. These stations are described below and listed in table 1 to classify the sites by type of data collected.

(1) Continuous-record streamflow-A waterstage sensor and a recording instrument records water stage from base (or zero) flow to flood stage. Discharge measurements are made to define a stage-discharge relation. Data computed include flood discharges, flood stages, daily average flows, and flood hydrographs.

(2) Flood hydrograph (and partial record, streamflow)_A water-stage sensor and a recording instrument records water stage from an arbitrary minimum stage to flood stage. Discharge measurements are made to define a stage-discharge relation. Data computed include flood discharges, flood stages, and flood hydrographs.

(3) Partial record, stage only-A water-stage sensor and a recording instrument records water stage from an arbitrary minimum stage to flood stage. Data computed include annual maximum stages and flood stages. 


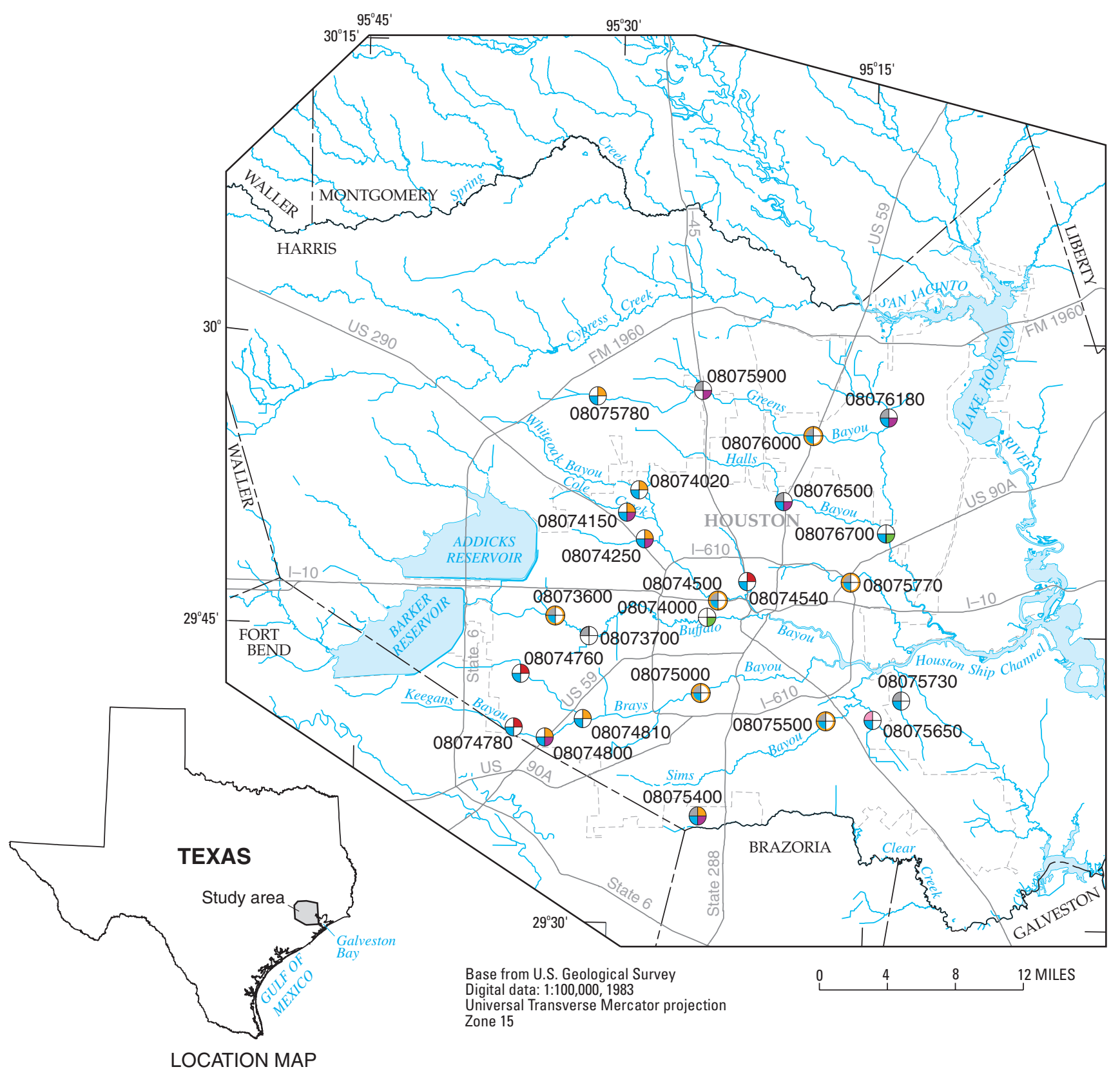

EXPLANATION

Type of U.S. Geological Survey station and number-Number corresponds to station number in table 1

08073700

$\oplus \quad$ Continuous-record streamflow station

08075650

$\oplus$ Continuous-record gage height station

$08074020 \begin{aligned} & \text { Partial record, annual maximum peak streamflow } \\ & \text { station }\end{aligned}$ 08074540

$\theta$

$\begin{array}{cl}08074150 & \begin{array}{l}\text { Partial record, peak streamflow greater than a base } \\ \text { discharge station }\end{array} \\ 08074000 & \begin{array}{l}\text { Flood hydrograph, continuous-record streamflow greater } \\ \text { than a base discharge station }\end{array} \\ 08076700 & \text { Precipitation station } \\ 08073600 & \text { Water-quality station }\end{array}$

Figure 1. Location of data-collection sites in the Houston metropolitan area, Texas. 
(4) Precipitation-Rainfall is collected with an instrument requiring either a tipping bucket or weighing device to determine accumulated rainfall. Data include incremental amounts for given time intervals. Rain gages used in this study are maintained by the HCOEM.

(5) Water quality - Water samples are collected manually. Samples are analyzed for physical, biological, and chemical properties and constituents.

\section{SUMMARY OF PRECIPITATION DATA}

All precipitation data measured in the study area are rainfall. Rainfall was measured by HCOEM gages that are colocated with USGS gaging stations (table 2, at end of report). These data are the basis for defining storm rainfall for the storm-hydrograph data presented in this report. Average annual rainfall from gages in the network ranged from a minimum of 34.54 inches (in.) during the 1990 water year to a maximum of 68.15 in. during the 1992 water year. The maximum daily rainfall amounts were measured in the study area during two storms: (1) March 4, 1992, when 6 stations within a narrow band on the upper Whiteoak, Greens, Garners, and Halls bayous measured between 5.98 and 7.80 in.; and (2) October 17-18, 1994, when 10 stations on several bayous (including Little Whiteoak, Keegans, Berry, Sims, Brays, Vince, Hunting, and Greens) throughout the study area measured as much as $10.16 \mathrm{in}$.

The precipitation data provided for water years 1990-95 are incremental amounts during selected storms (included on the $\mathrm{CD}$ with this report); data formats are described in a later section. A complete list of all precipitation data files is given in appendix 1.

The maximum recorded incremental rainfall observed during water years 1990-95 was $2.99 \mathrm{in}$. for 15-minute intervals, 3.27 in. for 30-minute intervals, and 4.28 in. for 60 -minute intervals. The maximum rainfall for the HURP studies during water years 196489 and water years 1990-95 can be compared to values for the 25- and 50-year recurrence intervals determined for the Houston area by the National Weather Service (NWS) (Frederick and others, 1977; Hershfield, 1961) and by the USGS in a more recent report (Asquith, 1998):

\begin{tabular}{|c|c|c|c|c|c|c|}
\hline \multirow[b]{2}{*}{$\begin{array}{c}\text { Time } \\
\text { increment } \\
\text { (minutes) }\end{array}$} & \multicolumn{2}{|c|}{ HURP } & \multicolumn{2}{|c|}{ NWS } & \multicolumn{2}{|c|}{ USGS } \\
\hline & $\begin{array}{c}\text { Maximum rainfall, } \\
\text { water years } \\
1964-89 \\
\text { (inches) }\end{array}$ & $\begin{array}{c}\text { Maximum rainfall, } \\
\text { water years } \\
1990-95 \\
\text { (inches) }\end{array}$ & $\begin{array}{c}\text { 25-year recurrence } \\
\text { interval total } \\
\text { rainfall } \\
\text { (inches) }\end{array}$ & $\begin{array}{c}\text { 50-year recurrence } \\
\text { interval total } \\
\text { rainfall } \\
\text { (inches) }\end{array}$ & $\begin{array}{c}\text { 25-year recurrence } \\
\text { interval total } \\
\text { rainfall } \\
\text { (inches) }\end{array}$ & $\begin{array}{c}\text { 50-year recurrence } \\
\text { interval total } \\
\text { rainfall } \\
\text { (inches) }\end{array}$ \\
\hline 15 & 2.25 & 2.99 & 1.7 & 1.9 & 1.7 & 1.9 \\
\hline 30 & 3.00 & 3.27 & 3.1 & 3.4 & 2.4 & 2.6 \\
\hline 60 & 4.90 & 4.28 & 3.8 & 4.2 & 3.4 & 3.8 \\
\hline
\end{tabular}

\section{SUMMARY OF SURFACE-WATER HYDROLOGIC DATA}

Two general types of data for water years 1990 95 are provided: (1) water-quantity data and (2) waterquality data. All data summarized in this section are on the $\mathrm{CD}$ included with this report; data formats are explained in a later section.

\section{Water-Quantity Data}

Water-quantity data are based on discharge measurements and stage records that were collected at 24 stations during the study (fig. 1, table 1). The number of continous-record streamflow-gaging stations ranged from 8 in water years $1994-95$ to 12 in water years 1990-91. The number of partial record stations ranged from 9 in water years $1990-91$ to 13 in water years 1994-95.

Data that describe surface-water conditions include the following:

1. Flood peaks-Annual flood peaks, defined as the maximum discharge in cubic feet per second (or the maximum stage in feet) that occurred during the water year, were determined for each station (table 3, at end of report). All stations had 10 or more years of record. During the 1990-95 water years, peak flows and (or) peak stages occurred at 12 stations during the March 1992 storm. These stations are located in the Buffalo, Whiteoak, Brays, and Garners Bayou Basins. One other peak flow measured in the study area during the 1990-95 water years occurred during the October 1994 storm. The analysis of flood peaks provides information for planners, engineers, and others to develop methods to understand, prevent, and (or) diminish potential damages caused by floods in the future.

2. Storm hydrographs - Discharge hydrographs (and for some stations, stage hydrographs) were determined for a selected number of storms during each water year at 23 stations (table 4, at end 
of report). A complete list of all discharge and stage data files are given in appendix 2 .

3. Daily mean flows-Daily mean discharges were computed for all continuous-record streamflow stations, including those where discharges were computed over the entire range of stage and those where discharges were calculated only above a specified base flow.

\section{Water-Quality Data}

Water-quality data, including physical, biological, and chemical properties and constituents, have been collected to represent various flow conditions during a water year. Analyses include properties (temperature, specific conductance, dissolved oxygen, $\mathrm{pH}$, color, turbidity, alkalinity, and biochemical oxygen demand), dissolved solids, bacteria, carbon, nutrients, major ions, trace elements, and pesticides. These data are included on the CD.

During water years 1990-95, 134 water-quality samples were collected at six stations (table 5, at end of report) by USGS personnel. These stations are located on each of the five major drainage basins (Buffalo, Whiteoak, Brays, Sims, and Greens Bayous) in the study area, as well as Hunting Bayou. Except for samples collected from Buffalo Bayou, samples were collected five times during each of the 1990 and 1991 water years with a strong emphasis on changes in chemical and biological conditions during the spring and summer months. During each of the 1992-95 water years, samples were collected three times-in February, May, and August.

Samples were analyzed at the USGS National Water Quality Laboratory for about 80 water-quality properties and constituents (table 6 , at end of report). For some constituents (such as nutrients and selected pesticides), either total or dissolved fractions were analyzed. At the beginning of the 1993 water year, analyses for total concentrations of nitrogen and phosphorus species were discontinued, and only the dissolved concentrations were reported. Summary statistics indicate the variation of water-quality properties and constituents in the study area during water years 1990-95.

\section{DATA AVAILABILITY AND FORMATS}

The data for this study are on a CD. The four directories on the $\mathrm{CD}$ indicate the type of data files available:

(1) stations, which contains station manuscripts for the 24 USGS streamflow-gaging stations for which flow and (or) stage data are provided; (2) sw, which contains data files of daily mean discharges for 14 continuousrecord streamflow stations (table 1), data files of peak flows and (or) stages for 24 stations, and data files of discharges or stages for selected storms for 23 stations; (3) precip, which contains data files of precipitation for 21 stations (table 1); and (4) qw, which contains data files of water quality for 6 stations (table 1). The types of data files are described below.

Station manuscript-The station manuscript provides pertinent information about the gaging station, including major river basin in which the station is located, station location, drainage area, period of record, gage datum, remarks, and stage/discharge extremes (for selected stations). These data files are ASCII files that are identified by a leading " $M$ " followed by the entire USGS station number. For example, the manuscript file for station 08074150 is M08074150.dat.

Daily mean discharge-Daily mean discharges are computed each year for all continuous-record streamflow stations. These data files are tab-delimited (.rdb) files. They are identified by a leading "QDV" followed by the last 5 digits of the USGS station number. For example, the daily mean discharge file for station 08074500 is QDV74500.rdb.

Peaks-Peaks are the maximum discharge and (or) stage that occurred during a water year at a station as well as peaks that exceed a base discharge, if computed. Data for each station are provided in tab-delimited files that are identified by a leading "PK" followed by the last 5 digits of the USGS station number. For example, available peaks for station 08074150 are contained in the file PK74150.rdb.

Storm hydrographs - Storm-hydrograph data include discharge or stage data for selected storms. These data files are tab-delimited files. Unit-value discharge data files are identified by a leading "quv" (unit-value stage data files are identified by a leading "ghuv") followed by the last 5 digits of the USGS station number and the month and year in which the storm began. For example, a unit-value discharge file that describes a storm in March 1992 recorded by station 08074150 is quv74150_0392.rdb.

Precipitation-Precipitation data comprise files of unit values during storms, coinciding with discharge data files. The data are provided in a tab-delimited format. For stations without colocated rain gages, precipitation data are included from stations with colocated rain gages in the watershed above the designated station. Precipitation data files are identified by a leading " $p$ " followed by the last 5 digits of the USGS station number and the beginning month and year of the data. For example, precipitation data for station 08074500 from a storm in March 1992 is p74500_0392.rdb. 
Water quality - Water-quality files provide data from water samples collected and analyzed for selected properties and constituents at a station. These data are provided in a tab-delimited format and arranged within the file by date sampled. Files are identified by a leading "QW" followed by the last 5 digits of the USGS station number. For example, the water-quality file for station 08074500 is QW74500.rdb.

\section{SUMMARY}

In 1964, the USGS began studies of streamflow in the Houston metropolitan area and in water year 1968, expanded HURP to include water quality. This report summarizes selected hydrologic data collected during water years 1990-95 and includes a CD of the complete database of stage, discharge, precipitation, and waterquality data.

Two general types of surface-water hydrologic data are presented for water years 1990-95: (1) waterquantity data and (2) water-quality data. Water-quantity data are based on discharge measurements and stage records collected at 24 streamflow-gaging stations; the data include annual peaks at the 24 stations (all with 10 or more years of record), storm hydrographs for selected storms during each water year at 23 stations, and daily mean flows at 14 stations. Samples were collected at six stations for water-quality analysis. About 80 physical, biological, and chemical properties and constituents were analyzed in 134 samples.

Precipitation data for water years 1990-95 are incremental amounts during selected storms. Rainfall was recorded by HCOEM gages that are colocated with USGS gaging stations. These data are the basis for defining storm rainfall for the storm-hydrograph data presented in this report.

\section{REFERENCES CITED}

Asquith, W.H., 1998, Depth-duration frequency of precipitation for Texas: U.S. Geological Survey Water-Resources Investigations Report 98-4044, 107 p.
Frederick, R.H., Myers, V.H., and Auciello, E.P., 1977, Fiveto 60-minute precipitation frequency for the eastern and central United States: Washington, D.C., National Oceanic and Atmospheric Administration Technical Memorandum NWS Hydro-35, 36 p.

Hershfield, D.M., 1961, Rainfall frequency atlas of the United States for durations from 30 minutes to 24 hours and return periods from 1 to 100 years: Washington, D.C., U.S. Weather Bureau Technical Paper 40, 61 p.

Johnson, S.L., and Sayre, D.M., 1973, Effects of urbanization on floods in the Houston, Texas, metropolitan area: U.S. Geological Survey Water-Resources Investigations Report 3-73, 50 p.

Liscum, Fred, 2001, Effects of urban development on stormwater runoff characteristics for the Houston, Texas, metropolitan area: U.S. Geological Survey WaterResources Investigations Report 01-4071, 35 p.

Liscum, Fred, Brown, D.W., and Kasmarek, M.C., 1997, Summary of surface-water hydrologic data for the Houston metropolitan area, Texas, water years 1964-89: U.S. Geological Survey Open-File Report 96-250, 44 p.

Liscum, Fred, and Massey, B.C., 1980, Technique for estimating the magnitude and frequency of floods in the Houston, Texas, metropolitan area: U.S. Geological Survey

Office of the Texas State Climatologist, 2002, Texas climatic bulletin: Accessed December 6, 2002, at URL http://www.met.tamu.edu/osc/tx/txyear.html

Real Estate Center at Texas A\&M University, 2002, Houston market overview 2002-Population: Accessed December 26, 2002, at URL

http://recenter.tamu.edu/mreports/houston2.asp

U.S. Army Corps of Engineers, 1976, Storage, treatment, overflow, runoff model "STORM": Davis, Calif., U.S. Army Corps of Engineers, Hydrologic Engineering Center, Computer Program Users Manual, 45 p.

U.S. Census Bureau, 2002, Population estimates, 1990s: Population Estimates Program, Population Division, accessed December 6, 2002, at URL http://eire.census.gov/popest/archives/1990.php 
Table 1. Type of data collected at U.S. Geological Survey streamflow-gaging stations during water years 1990-95 [X, data collected; --, data not collected]

\begin{tabular}{|c|c|c|c|c|c|c|c|c|}
\hline \multirow{2}{*}{$\begin{array}{c}\text { Station } \\
\text { no. } \\
\text { (fig. 1) }\end{array}$} & \multirow[b]{2}{*}{ Station name } & \multirow[b]{2}{*}{ Type of data collected } & \multicolumn{6}{|c|}{ Water year } \\
\hline & & & 1990 & 1991 & 1992 & 1993 & 1994 & 1995 \\
\hline \multirow{3}{*}{$\overline{08073600}$} & Buffalo Bayou at West Belt & Continuous-record streamflow & $\mathrm{X}$ & $\mathrm{X}$ & $\mathrm{X}$ & $\mathrm{X}$ & $\mathrm{X}$ & $\mathrm{X}$ \\
\hline & Dr., Houston, Tex. & Water quality & $\mathrm{X}$ & $\mathrm{X}$ & $\mathrm{X}$ & $\mathrm{X}$ & $\mathrm{X}$ & $\mathrm{X}$ \\
\hline & & Precipitation & $X$ & $\mathrm{X}$ & $\mathrm{X}$ & $\mathrm{X}$ & $\mathrm{X}$ & $\mathrm{X}$ \\
\hline 08073700 & $\begin{array}{l}\text { Buffalo Bayou at Piney } \\
\text { Point, Tex. }\end{array}$ & Continuous-record streamflow & $\mathrm{X}$ & $\mathrm{X}$ & $\mathrm{X}$ & $\mathrm{X}$ & $\mathrm{X}$ & $\mathrm{X}$ \\
\hline 08074000 & $\begin{array}{l}\text { Buffalo Bayou at Houston, } \\
\text { Tex. }\end{array}$ & $\begin{array}{l}\text { Flood hydrograph, continuous-record streamflow } \\
\text { greater than a base discharge }\end{array}$ & $\mathrm{X}$ & $\mathrm{X}$ & $\mathrm{X}$ & $\mathrm{X}$ & $\mathrm{X}$ & $\mathrm{X}$ \\
\hline \multirow[t]{2}{*}{08074020} & Whiteoak Bayou at Alabon- & Partial record, annual maximum peak streamflow & $\mathrm{X}$ & $\mathrm{X}$ & $\mathrm{X}$ & $\mathrm{X}$ & $\mathrm{X}$ & $\mathrm{X}$ \\
\hline & son Rd. at Houston, Tex. & Precipitation & $\mathrm{X}$ & $\mathrm{X}$ & $\mathrm{X}$ & $\mathrm{X}$ & $\mathrm{X}$ & $\mathrm{X}$ \\
\hline \multirow[t]{3}{*}{08074150} & Cole Creek at Deihl Rd., & Partial record, annual maximum peak streamflow & $\mathrm{X}$ & $\mathrm{X}$ & $\mathrm{X}$ & -- & -- & -- \\
\hline & Houston, Tex. & $\begin{array}{l}\text { Partial record, peak streamflow greater than a } \\
\text { base discharge }\end{array}$ & -- & -- & -- & $\mathrm{X}$ & $\mathrm{X}$ & $\mathrm{X}$ \\
\hline & & Precipitation & $\mathrm{X}$ & $\mathrm{X}$ & $\mathrm{X}$ & $\mathrm{X}$ & $\mathrm{X}$ & $\mathrm{X}$ \\
\hline \multirow[t]{3}{*}{08074250} & Brickhouse Gully at Costa & Partial record, annual maximum peak streamflow & $\mathrm{X}$ & $\mathrm{X}$ & $\mathrm{X}$ & -- & -- & -- \\
\hline & Rica St., Houston, Tex. & $\begin{array}{l}\text { Partial record, peak streamflow greater than a } \\
\text { base discharge }\end{array}$ & -- & -- & -- & $\mathrm{X}$ & $\mathrm{X}$ & $\mathrm{X}$ \\
\hline & & Precipitation & $\mathrm{X}$ & $\mathrm{X}$ & $\mathrm{X}$ & $\mathrm{X}$ & $\mathrm{X}$ & $\mathrm{X}$ \\
\hline \multirow[t]{3}{*}{08074500} & Whiteoak Bayou at & Continuous-record streamflow & $\mathrm{X}$ & $\mathrm{X}$ & $\mathrm{X}$ & $\mathrm{X}$ & $\mathrm{X}$ & $\mathrm{X}$ \\
\hline & Houston, Tex. & Water quality & $\mathrm{X}$ & $\mathrm{X}$ & $\mathrm{X}$ & $\mathrm{X}$ & $\mathrm{X}$ & $\mathrm{X}$ \\
\hline & & Precipitation & $\mathrm{X}$ & $\mathrm{X}$ & $\mathrm{X}$ & $\mathrm{X}$ & $\mathrm{X}$ & $\mathrm{X}$ \\
\hline \multirow[t]{2}{*}{08074540} & Little Whiteoak Bayou at & Partial record, annual maximum gage height & $\mathrm{X}$ & $\mathrm{X}$ & $\mathrm{X}$ & $\mathrm{X}$ & $\mathrm{X}$ & $\mathrm{X}$ \\
\hline & $\begin{array}{l}\text { Trimble St. at Houston, } \\
\text { Tex. }\end{array}$ & Precipitation & $\mathrm{X}$ & $\mathrm{X}$ & $\mathrm{X}$ & $\mathrm{X}$ & $\mathrm{X}$ & $\mathrm{X}$ \\
\hline \multirow[t]{2}{*}{08074760} & Brays Bayou at Alief, Tex. & Partial record, annual maximum gage height & $\mathrm{X}$ & $\mathrm{X}$ & $\mathrm{X}$ & $\mathrm{X}$ & $\mathrm{X}$ & $\mathrm{X}$ \\
\hline & & Precipitation & $\mathrm{X}$ & $\mathrm{X}$ & $\mathrm{X}$ & $\mathrm{X}$ & $\mathrm{X}$ & $\mathrm{X}$ \\
\hline \multirow[t]{2}{*}{08074780} & Keegans Bayou at Keegan & Partial record, annual maximum gage height & $\mathrm{X}$ & $\mathrm{X}$ & $\mathrm{X}$ & $\mathrm{X}$ & $\mathrm{X}$ & $\mathrm{X}$ \\
\hline & Rd. near Houston, Tex. & Precipitation & $\mathrm{X}$ & $\mathrm{X}$ & $\mathrm{X}$ & $\mathrm{X}$ & $\mathrm{X}$ & $\mathrm{X}$ \\
\hline \multirow[t]{3}{*}{08074800} & Keegans Bayou at Roark & Partial record, annual maximum peak streamflow & $\mathrm{X}$ & $\mathrm{X}$ & $\mathrm{X}$ & -- & -- & -- \\
\hline & Rd. near Houston, Tex. & $\begin{array}{l}\text { Partial record, peak streamflow greater than a } \\
\text { base discharge }\end{array}$ & -- & -- & -- & $\mathrm{X}$ & $\mathrm{X}$ & $\mathrm{X}$ \\
\hline & & Precipitation & $\mathrm{X}$ & $\mathrm{X}$ & $\mathrm{X}$ & $\mathrm{X}$ & $\mathrm{X}$ & $\mathrm{X}$ \\
\hline \multirow[t]{2}{*}{08074810} & Brays Bayou at Gessner & Partial record, annual maximum peak streamflow & $\mathrm{X}$ & $\mathrm{X}$ & $\mathrm{X}$ & $\mathrm{X}$ & $\mathrm{X}$ & $\mathrm{X}$ \\
\hline & Dr., Houston, Tex. & Precipitation & $\mathrm{X}$ & $\mathrm{X}$ & $\mathrm{X}$ & $\mathrm{X}$ & $\mathrm{X}$ & $\mathrm{X}$ \\
\hline \multirow[t]{3}{*}{08075000} & Brays Bayou at Houston, & Continuous-record streamflow & $\mathrm{X}$ & $\mathrm{X}$ & $\mathrm{X}$ & $\mathrm{X}$ & $\mathrm{X}$ & $\mathrm{X}$ \\
\hline & Tex. & Water quality & $\mathrm{X}$ & $\mathrm{X}$ & $\mathrm{X}$ & $\mathrm{X}$ & $\mathrm{X}$ & $\mathrm{X}$ \\
\hline & & Precipitation & $\mathrm{X}$ & $\mathrm{X}$ & $\mathrm{X}$ & $\mathrm{X}$ & $\mathrm{X}$ & $\mathrm{X}$ \\
\hline
\end{tabular}


Table 1. Type of data collected at U.S. Geological Survey streamflow-gaging stations during water years 199095-Continued

\begin{tabular}{|c|c|c|c|c|c|c|c|c|}
\hline \multirow{2}{*}{$\begin{array}{c}\text { Station } \\
\text { no. } \\
\text { (fig. 1) }\end{array}$} & \multirow[b]{2}{*}{ Station name } & \multirow[b]{2}{*}{ Type of data collected } & \multicolumn{6}{|c|}{ Water year } \\
\hline & & & 1990 & 1991 & 1992 & 1993 & 1994 & 1995 \\
\hline \multirow[t]{4}{*}{08075400} & Sims Bayou at Hiram & Continuous-record streamflow & $\mathrm{X}$ & $\mathrm{X}$ & -- & -- & -- & -- \\
\hline & Clarke St., Houston, Tex. & Partial record, annual maximum peak streamflow & -- & -- & $\mathrm{X}$ & -- & -- & -- \\
\hline & & $\begin{array}{l}\text { Partial record, peak streamflow greater than a } \\
\text { base discharge }\end{array}$ & -- & -- & -- & $\mathrm{X}$ & $\mathrm{X}$ & $\mathrm{X}$ \\
\hline & & Precipitation & $\mathrm{X}$ & $\mathrm{X}$ & $\mathrm{X}$ & $\mathrm{X}$ & $\mathrm{X}$ & $\mathrm{X}$ \\
\hline \multirow[t]{3}{*}{08075500} & Sims Bayou at Houston, & Continuous-record streamflow & $\mathrm{X}$ & $\mathrm{X}$ & $\mathrm{X}$ & $\mathrm{X}$ & $\mathrm{X}$ & $\mathrm{X}$ \\
\hline & Tex. & Water quality & $\mathrm{X}$ & $\mathrm{X}$ & $\mathrm{X}$ & $\mathrm{X}$ & $\mathrm{X}$ & $\mathrm{X}$ \\
\hline & & Precipitation & $\mathrm{X}$ & $\mathrm{X}$ & $\mathrm{X}$ & $\mathrm{X}$ & $\mathrm{X}$ & $\mathrm{X}$ \\
\hline \multirow[t]{2}{*}{08075650} & Berry Bayou at Forest Oaks & Continuous-record gage height & $\mathrm{X}$ & $\mathrm{X}$ & $\mathrm{X}$ & $\mathrm{X}$ & $\mathrm{X}$ & $\mathrm{X}$ \\
\hline & St., Houston, Tex. & Precipitation & $\mathrm{X}$ & $\mathrm{X}$ & $\mathrm{X}$ & $\mathrm{X}$ & $\mathrm{X}$ & $\mathrm{X}$ \\
\hline \multirow[t]{2}{*}{08075730} & Vince Bayou at Pasadena, & Continuous-record streamflow & $\mathrm{X}$ & $\mathrm{X}$ & $\mathrm{X}$ & $\mathrm{X}$ & $\mathrm{X}$ & $\mathrm{X}$ \\
\hline & Tex. & Precipitation & $\mathrm{X}$ & $\mathrm{X}$ & $\mathrm{X}$ & $\mathrm{X}$ & $\mathrm{X}$ & $\mathrm{X}$ \\
\hline \multirow{3}{*}{08075770} & Hunting Bayou at IH-610, & Continuous-record streamflow & $\mathrm{X}$ & $\mathrm{X}$ & $\mathrm{X}$ & $\mathrm{X}$ & $\mathrm{X}$ & $\mathrm{X}$ \\
\hline & Houston, Tex. & Water quality & $\mathrm{X}$ & $\mathrm{X}$ & $\mathrm{X}$ & $\mathrm{X}$ & $\mathrm{X}$ & $\mathrm{X}$ \\
\hline & & Precipitation & $\mathrm{X}$ & $\mathrm{X}$ & $\mathrm{X}$ & $\mathrm{X}$ & $\mathrm{X}$ & $\mathrm{X}$ \\
\hline \multirow[t]{2}{*}{08075780} & Greens Bayou at Cutten Rd. & Partial record, annual maximum peak streamflow & $\mathrm{X}$ & $\mathrm{X}$ & $\mathrm{X}$ & $\mathrm{X}$ & $\mathrm{X}$ & $\mathrm{X}$ \\
\hline & near Houston, Tex. & Precipitation & $\mathrm{X}$ & $\mathrm{X}$ & $\mathrm{X}$ & $\mathrm{X}$ & $\mathrm{X}$ & $\mathrm{X}$ \\
\hline \multirow[t]{2}{*}{08075900} & Greens Bayou near U.S. & Continuous-record streamflow & $\mathrm{X}$ & $\mathrm{X}$ & $X$ & -- & -- & -- \\
\hline & $\begin{array}{l}\text { Highway } 75 \text { near } \\
\text { Houston, Tex. }\end{array}$ & $\begin{array}{l}\text { Partial record, peak streamflow greater than a } \\
\text { base discharge }\end{array}$ & -- & -- & -- & $\mathrm{X}$ & $\mathrm{X}$ & $\mathrm{X}$ \\
\hline \multirow[t]{3}{*}{08076000} & Greens Bayou near & record streamflow & $\mathrm{X}$ & $\mathrm{X}$ & $\mathrm{X}$ & $\mathrm{X}$ & $\mathrm{X}$ & $\mathrm{X}$ \\
\hline & Houston, Tex. & Water quality & $\mathrm{X}$ & $\mathrm{X}$ & $\mathrm{X}$ & $\mathrm{X}$ & $\mathrm{X}$ & $\mathrm{X}$ \\
\hline & & Precipitation & $\mathrm{X}$ & $\mathrm{X}$ & $\mathrm{X}$ & $X$ & $X$ & $\mathrm{X}$ \\
\hline \multirow[t]{3}{*}{08076180} & Garners Bayou near & Contin & $\mathrm{X}$ & $\mathrm{X}$ & $X$ & $\mathrm{X}$ & -- & -- \\
\hline & Humble, Tex. & $\begin{array}{l}\text { Partial record, peak streamflow greater than a } \\
\text { base discharge }\end{array}$ & -- & -- & -- & -- & $\mathrm{X}$ & $\mathrm{X}$ \\
\hline & & Precipitation & $\mathrm{X}$ & $\mathrm{X}$ & $\mathrm{X}$ & $\mathrm{X}$ & $\mathrm{X}$ & $\mathrm{X}$ \\
\hline \multirow[t]{3}{*}{08076500} & Halls Bayou at Houston, & Continuous-record streamflow & $\mathrm{X}$ & $\mathrm{X}$ & $\mathrm{X}$ & $\mathrm{X}$ & -- & -- \\
\hline & Tex. & $\begin{array}{l}\text { Partial record, peak streamflow greater than a } \\
\text { base discharge }\end{array}$ & -- & -- & -- & -- & $\mathrm{X}$ & $\mathrm{X}$ \\
\hline & & Precipitation & $\mathrm{X}$ & $\mathrm{X}$ & $\mathrm{X}$ & $\mathrm{X}$ & $\mathrm{X}$ & $\mathrm{X}$ \\
\hline \multirow[t]{2}{*}{08076700} & $\begin{array}{l}\text { Greens Bayou at Ley Rd., } \\
\text { Houston, Tex. }\end{array}$ & $\begin{array}{l}\text { Flood hydrograph, continuous-record streamflow } \\
\text { greater than a base discharge }\end{array}$ & $\mathrm{X}$ & $\mathrm{X}$ & -- & $\mathrm{X}$ & $\mathrm{X}$ & $\mathrm{X}$ \\
\hline & & Precipitation & $\mathrm{X}$ & $\mathrm{X}$ & $\mathrm{X}$ & $\mathrm{X}$ & $\mathrm{X}$ & $\mathrm{X}$ \\
\hline
\end{tabular}


Table 2. Description of Harris County Office of Emergency Management rain gages used to provide data for this study

\begin{tabular}{clrl}
\hline $\begin{array}{c}\text { USGS station no. } \\
\text { (fig. 1) }\end{array}$ & \multicolumn{1}{c}{ Station name } & $\begin{array}{c}\text { HCOEM } \\
\text { station ID }\end{array}$ & $\begin{array}{c}\text { Period of } \\
\text { record }^{1}\end{array}$ \\
\hline 08073600 & Buffalo Bayou at West Belt Dr., Houston, Tex. & 2270 & May 1988-current \\
08074020 & Whiteoak Bayou at Alabonson Rd. at Houston, Tex. & 540 & July 1984-current \\
08074150 & Cole Creek at Deihl Rd., Houston, Tex. & 590 & Apr. 1984-current \\
08074250 & Brickhouse Gully at Costa Rica St., Houston, Tex. & 580 & Apr. 1984-current \\
08074500 & Whiteoak Bayou at Houston, Tex. & 520 & Mar. 1984-current \\
08074540 & Little Whiteoak Bayou at Trimble St. at Houston, Tex. & 560 & May 1984-current \\
08074760 & Brays Bayou at Alief, Tex. & 470 & May 1984-current \\
08074780 & Keegans Bayou at Keegan Rd. near Houston, Tex. & 490 & May 1984-current \\
08074800 & Keegans Bayou at Roark Rd. near Houston, Tex. & 480 & Apr. 1984-current \\
08074810 & Brays Bayou at Gessner Dr., Houston, Tex. & 460 & Apr. 1984-current \\
08075000 & Brays Bayou at Houston, Tex. & 420 & Apr. 1984-current \\
08075400 & Sims Bayou at Hiram Clarke St., Houston, Tex. & 380 & June 1983-current \\
08075500 & Sims Bayou at Houston, Tex. & 340 & June 1983-current \\
08075650 & Berry Bayou at Forest Oaks St., Houston, Tex. & 320 & June 1983-current \\
08075730 & Vince Bayou at Pasadena, Tex. & 920 & Feb. 1984-current \\
08075770 & Hunting Bayou at IH-610, Houston, Tex. & 830 & Oct. 1985-current \\
08075780 & Greens Bayou at Cutten Rd. near Houston, Tex. & 1670 & Mar. 1986-current \\
08076000 & Greens Bayou near U.S. Highway 75 near Houston, Tex. & 1640 & May 1984-current \\
08076180 & Garners Bayou near Humble, Tex. & 1630 & July 1984-current \\
08076500 & Halls Bayou at Houston, Tex. & 1680 & May 1984-current \\
08076700 & Greens Bayou at Ley Rd., Houston, Tex. & 1620 & June 1983-current \\
\hline
\end{tabular}

${ }^{1}$ Data not available for all stations, Nov. 1-30, 1994. 
Table 3. Observed annual peak discharges for U.S. Geological Survey streamflow-gaging stations during water years 1990-95

[In cubic feet per second except where noted. Past peak discharge, maximum for period of USGS data collection prior to 1990; record peak discharge, maximum for period of record; --, not determined]

\begin{tabular}{|c|c|c|c|c|c|c|c|c|}
\hline \multirow{2}{*}{$\begin{array}{c}\text { Station } \\
\text { no. }\end{array}$} & \multirow{2}{*}{$\begin{array}{c}\text { Past } \\
\text { peak discharge } \\
\text { (date) }\end{array}$} & \multicolumn{6}{|c|}{ Water year peak discharge } & \multirow{2}{*}{$\begin{array}{l}\text { Record peak } \\
\text { discharge }^{1} \\
\text { (date) }\end{array}$} \\
\hline & & 1990 & 1991 & 1992 & 1993 & 1994 & 1995 & \\
\hline 08073600 & $\begin{array}{l}5,350 \\
(08-31-81)\end{array}$ & 2,320 & 2,530 & 7,290 & 3,680 & 2,150 & 3,780 & $\begin{array}{c}7,290 \\
(03-04-92)\end{array}$ \\
\hline 08073700 & $\begin{array}{l}5,700 \\
(08-31-81)\end{array}$ & 2,280 & 2,580 & 7,500 & 4,550 & 2,570 & 4,940 & $\begin{array}{c}7,500 \\
(03-04-92)\end{array}$ \\
\hline 08074000 & $\begin{array}{l}10,900 \\
(08-30-45)\end{array}$ & 4,520 & 4,840 & 12,500 & 6,710 & 5,890 & 8,450 & $\begin{array}{l}12,500 \\
(03-04-92)\end{array}$ \\
\hline 08074020 & $\begin{array}{l}7,390 \\
(05-18-89)\end{array}$ & 3,420 & 4,440 & 8,610 & 4,520 & 1,890 & 3,980 & $\begin{array}{l}8,610 \\
(03-04-92)\end{array}$ \\
\hline 08074150 & $\begin{array}{l}1,900 \\
(05-18-89)\end{array}$ & 1,120 & 1,180 & 2,780 & 954 & 598 & 773 & $\begin{array}{l}2,780 \\
(03-04-92)\end{array}$ \\
\hline 08074250 & $\begin{array}{l}5,800 \\
(03-20-72)\end{array}$ & 3,130 & 4,540 & 7,580 & 3,320 & 3,800 & 2,700 & $\begin{array}{l}7,580 \\
(03-04-92)\end{array}$ \\
\hline 08074500 & $\begin{array}{l}18,300 \\
(06-26-89)\end{array}$ & 8,550 & 9,310 & 25,100 & 11,200 & 6,850 & 11,800 & $\begin{array}{l}25,100 \\
(03-04-92)\end{array}$ \\
\hline 08074540 & $\begin{array}{c}240.25 \\
(10-25-84)\end{array}$ & ${ }^{2} 33.84$ & ${ }^{2} 34.48$ & ${ }^{2} 43.17$ & ${ }^{2} 38.25$ & ${ }^{2} 36.54$ & ${ }^{2} 39.29$ & $\begin{array}{c}243.17 \\
(03-04-92)\end{array}$ \\
\hline 08074760 & $\begin{array}{c}4,580 \\
(08-31-81) \\
219.59 \\
(08-31-81)\end{array}$ & ${ }^{2} 14.41$ & ${ }^{2} 13.40$ & ${ }^{2} 21.16$ & ${ }^{2} 14.41$ & ${ }^{2} 12.30$ & ${ }^{2} 16.83$ & $\begin{array}{c}{ }^{2} 21.16 \\
(03-04-92)\end{array}$ \\
\hline 08074780 & $\begin{array}{c}283.53 \\
(04-14-66)\end{array}$ & 275.38 & ${ }^{2} 76.71$ & ${ }^{2} 80.93$ & ${ }^{2} 78.11$ & 276.14 & ${ }^{2} 80.27$ & $\begin{array}{c}283.53 \\
(04-14-66)\end{array}$ \\
\hline 08074800 & $\begin{array}{l}4,250 \\
(09-19-83)\end{array}$ & 1,670 & 2,500 & 4,880 & 3,000 & 2,410 & 4,850 & $\begin{array}{l}4,880 \\
(03-04-92)\end{array}$ \\
\hline 08074810 & $\begin{array}{l}16,800 \\
(09-19-83)\end{array}$ & 3,690 & 9,470 & 16,900 & 8,380 & 7,900 & 11,000 & $\begin{array}{l}16,900 \\
(03-04-92)\end{array}$ \\
\hline 08075000 & $\begin{array}{l}29,000 \\
(06-15-76, \\
09-19-83)\end{array}$ & 10,400 & 19,800 & 23,000 & 16,000 & 16,500 & 27,000 & $\begin{array}{l}29,000 \\
(06-15-76, \\
09-19-83)\end{array}$ \\
\hline 08075400 & $\begin{array}{l}5,190 \\
(08-01-89)\end{array}$ & 875 & 5,120 & 6,290 & 4,540 & 3,710 & 7,510 & $\begin{array}{l}7,510 \\
(10-18-94)\end{array}$ \\
\hline 08075500 & $\begin{array}{l}11,400 \\
(08-18-83)\end{array}$ & 1,680 & 7,040 & 4,490 & 4,180 & 4,770 & 7,730 & $\begin{array}{l}11,400 \\
(08-18-83)\end{array}$ \\
\hline 08075650 & $\begin{array}{c}5,080 \\
(06-09-75) \\
223.85 \\
(09-20-79)\end{array}$ & ${ }^{2} 10.54$ & ${ }^{2} 16.44$ & ${ }^{2} 14.17$ & ${ }^{2} 12.07$ & ${ }^{2} 15.61$ & ${ }^{2} 17.97$ & $\begin{array}{c}5,080 \\
(06-09-75) \\
223.85 \\
(09-20-79)\end{array}$ \\
\hline
\end{tabular}

Footnotes at end of table 
Table 3. Observed annual peak discharges for U.S. Geological Survey streamflow-gaging stations during water years 1990-95-Continued

\begin{tabular}{|c|c|c|c|c|c|c|c|c|}
\hline \multirow{2}{*}{$\begin{array}{c}\text { Station } \\
\text { no. }\end{array}$} & \multirow{2}{*}{$\begin{array}{c}\text { Past } \\
\text { peak discharge } \\
\text { (date) }\end{array}$} & \multicolumn{6}{|c|}{ Water year peak discharge } & \multirow{2}{*}{$\begin{array}{l}\text { Record peak } \\
\text { discharge }^{1} \\
\text { (date) }\end{array}$} \\
\hline & & 1990 & 1991 & 1992 & 1993 & 1994 & 1995 & \\
\hline 08075730 & $\begin{array}{l}4,720 \\
(05-03-81)\end{array}$ & 2,170 & 2,210 & 1,830 & 1,750 & 2,030 & 2,710 & $\begin{array}{l}4,720 \\
(05-03-81)\end{array}$ \\
\hline 08075770 & $\begin{array}{l}3,470 \\
(06-26-89)\end{array}$ & 850 & 1,900 & 2,800 & 2,890 & 1,970 & 3,430 & $\begin{array}{l}3,470 \\
(06-26-89)\end{array}$ \\
\hline 08075780 & $\begin{array}{l}2,110 \\
(10-25-84)\end{array}$ & 552 & 889 & 904 & 715 & 740 & 962 & $\begin{array}{l}2,110 \\
(10-25-84)\end{array}$ \\
\hline 08075900 & $\begin{array}{l}13,000 \\
(06-26-89)\end{array}$ & 2,930 & 4,820 & 8,180 & 5,500 & 1,940 & 6,150 & $\begin{array}{l}13,000 \\
(06-26-89)\end{array}$ \\
\hline 08076000 & $\begin{array}{l}16,500 \\
(06-27-89)\end{array}$ & 2,810 & 4,280 & 9,560 & 5,620 & 3,150 & 7,150 & $\begin{array}{l}16,500 \\
(06-27-89)\end{array}$ \\
\hline 08076180 & $\begin{array}{c}8,030 \\
(06-27-89)\end{array}$ & 1,540 & 2,610 & 9,980 & 3,900 & 2,370 & 3,940 & $\begin{array}{c}9,980 \\
(03-04-92)\end{array}$ \\
\hline 08076500 & $\begin{array}{l}5,000 \\
(06-27-89)\end{array}$ & 1,120 & 1,890 & 4,840 & 2,650 & 1,940 & 2,580 & $\begin{array}{l}5,000 \\
(06-27-89)\end{array}$ \\
\hline 08076700 & $\begin{array}{l}32,500 \\
(06-27-89)\end{array}$ & 5,220 & 8,490 & -- & 13,700 & 8,840 & 21,800 & $\begin{array}{l}32,500 \\
(06-27-89)\end{array}$ \\
\hline
\end{tabular}

${ }^{1} 10$ or more years of record.

${ }^{2}$ Gage height in feet above datum.

Table 4. Summary of storm-hydrograph data for U.S. Geological Survey streamflow-gaging stations during water years 1990-95

[ft ${ }^{3} / \mathrm{s}$, cubic feet per second; ft, feet; --, not determined]

\begin{tabular}{|c|c|c|c|c|c|c|c|}
\hline \multirow{2}{*}{$\begin{array}{c}\text { Station } \\
\text { no. }\end{array}$} & \multirow{2}{*}{$\begin{array}{c}\text { Total } \\
\text { no. of } \\
\text { storms }\end{array}$} & \multicolumn{2}{|c|}{ Maximum, all storms } & \multirow{2}{*}{$\begin{array}{c}\text { Station } \\
\text { no. }\end{array}$} & \multirow{2}{*}{$\begin{array}{c}\text { Total } \\
\text { no. of } \\
\text { storms }\end{array}$} & \multicolumn{2}{|c|}{ Maximum, all storms } \\
\hline & & $\begin{array}{c}\text { Discharge } \\
\left(\mathrm{ft}^{3} / \mathrm{s}\right)\end{array}$ & $\begin{array}{c}\text { Stage } \\
\text { (ft) }\end{array}$ & & & $\begin{array}{c}\text { Discharge } \\
\left(\mathrm{ft}^{3} / \mathrm{s}\right)\end{array}$ & $\begin{array}{c}\text { Stage } \\
\text { (ft) }\end{array}$ \\
\hline 08073700 & 19 & 7,500 & 61.23 & 08075400 & 17 & 7,510 & 54.65 \\
\hline 08074000 & 20 & 12,500 & 34.63 & 08075500 & 18 & 7,730 & 29.88 \\
\hline 08074020 & 25 & 4,520 & 45.87 & 08075650 & 12 & -- & 17.97 \\
\hline 08074150 & 23 & 2,780 & 80.73 & 08075730 & 19 & 2,710 & 16.00 \\
\hline 08074250 & 26 & 7,580 & 71.26 & 08075770 & 20 & 3,430 & 39.80 \\
\hline 08074500 & 24 & 25,100 & 50.43 & 08075780 & 20 & 904 & 114.22 \\
\hline 08074540 & 11 & -- & 43.17 & 08075900 & 21 & 8,180 & 86.39 \\
\hline 08074760 & 21 & -- & 21.16 & 08076000 & 22 & 9,560 & 64.15 \\
\hline 08074780 & 19 & -- & 80.93 & 08076180 & 22 & 9,980 & 57.27 \\
\hline 08074800 & 20 & 4,880 & 75.91 & 08076500 & 21 & 4,840 & 62.72 \\
\hline 08074810 & 23 & 16,900 & 65.42 & 08076700 & 14 & 21,800 & 36.10 \\
\hline 08075000 & 23 & 27,000 & 51.02 & & & & \\
\hline
\end{tabular}


Table 5. Summary of samples collected at U.S. Geological Survey water-quality stations during water years 1990-95

\begin{tabular}{|c|c|c|c|c|c|c|}
\hline \multirow{2}{*}{$\begin{array}{c}\text { Station } \\
\text { no. }\end{array}$} & \multicolumn{6}{|c|}{ No. of samples collected during water year } \\
\hline & 1990 & 1991 & 1992 & 1993 & 1994 & 1995 \\
\hline 08073600 & 4 & 4 & 4 & 4 & 4 & 4 \\
\hline 08074500 & 5 & 5 & 3 & 3 & 3 & 3 \\
\hline 08075000 & 5 & 5 & 3 & 3 & 3 & 3 \\
\hline 08075500 & 5 & 5 & 3 & 3 & 3 & 3 \\
\hline 08075770 & 5 & 5 & 3 & 3 & 3 & 3 \\
\hline 08076000 & 5 & 5 & 3 & 3 & 3 & 3 \\
\hline
\end{tabular}

Table 6. Statistical summary of water-quality data collected at six stations during water years 1990-95

$\left[{ }^{\circ} \mathrm{C}\right.$, degrees Celsius; N/A, not applicable; $\mu \mathrm{S} / \mathrm{cm}$, microsiemens per centimeter at 25 degrees Celsius; $\mathrm{mg} / \mathrm{L}$; milligrams per liter; NTU, nephelometric turbidity units; <, less than; $\mu \mathrm{m}$, micrometer; cols. $/ 100 \mathrm{~mL}$, colonies per 100 milliliters; E, estimated; $\mu \mathrm{g} / \mathrm{L}$, micrograms per liter]

\begin{tabular}{|c|c|c|c|c|c|c|c|}
\hline \multirow{2}{*}{$\begin{array}{l}\text { Parameter } \\
\text { no. }\end{array}$} & \multirow{2}{*}{$\begin{array}{l}\text { Water-quality property or constituent } \\
\text { (unit) }\end{array}$} & \multirow{2}{*}{$\begin{array}{l}\text { No. of } \\
\text { sam- } \\
\text { ples }\end{array}$} & \multirow{2}{*}{$\begin{array}{l}\text { No. of } \\
\text { detec- } \\
\text { tions }\end{array}$} & \multirow{2}{*}{$\begin{array}{l}\text { Minimum } \\
\text { reporting } \\
\text { limit }\end{array}$} & \multicolumn{3}{|c|}{ Descriptive statistics } \\
\hline & & & & & Maximum & Minimum & Mean \\
\hline \multicolumn{8}{|c|}{ Physical properties } \\
\hline P00010 & Temperature, water $\left({ }^{\circ} \mathrm{C}\right)$ & 134 & N/A & N/A & 33.5 & 10.0 & 25.7 \\
\hline P00095 & Specific conductance $\left(\mu \mathrm{S} / \mathrm{cm}\right.$ at $\left.25^{\circ} \mathrm{C}\right)$ & 134 & N/A & N/A & 1,810 & 102 & 740 \\
\hline P00300 & Oxygen, dissolved (mg/L) & 134 & N/A & N/A & 19.0 & 2.5 & 7.5 \\
\hline P00400 & $\mathrm{pH}$, whole, field (standard units) & 133 & N/A & N/A & 8.9 & 6.4 & 7.9 \\
\hline P00080 & Color (platinum-cobalt units) & 134 & N/A & N/A & 400 & 1 & 22 \\
\hline P00076 & Turbidity (NTU) & 134 & N/A & N/A & 170 & 1.3 & 20 \\
\hline P39036 & Alkalinity, dissolved, fixed endpoint, field $\left(\mathrm{mg} / \mathrm{L}\right.$ as $\left.\mathrm{CaCO}_{3}\right)$ & 109 & N/A & N/A & 270 & 30 & 170 \\
\hline P00530 & Residue, total, nonfilterable (mg/L) & 134 & N/A & N/A & 430 & $<1$ & 28 \\
\hline P00535 & Residue, volatile, nonfilterable (mg/L) & 134 & N/A & N/A & 60 & $<1$ & 8 \\
\hline P00310 & Biochemical oxygen demand, 5-day at $20{ }^{\circ} \mathrm{C}(\mathrm{mg} / \mathrm{L})$ & 134 & N/A & N/A & 12.0 & .4 & 3.0 \\
\hline P80082 & Biochemical oxygen demand, carbonaceous, 5 -day at $20^{\circ} \mathrm{C}(\mathrm{mg} / \mathrm{L})$ & 133 & N/A & N/A & 8.1 & .3 & 2.3 \\
\hline \multicolumn{8}{|c|}{ Indicator bacteria } \\
\hline P31625 & Fecal coliform, $0.7-\mu \mathrm{m}$ membrane filter (cols. $/ 100 \mathrm{~mL}$ ) & 110 & 110 & N/A & 45,000 & E1 & 2,300 \\
\hline P31673 & Fecal streptococci, membrane filter, KF agar (cols./100 mL) & 110 & 110 & N/A & 27,000 & E1 & 260 \\
\hline
\end{tabular}


Table 6. Statistical summary of water-quality data collected at six stations during water years 1990-95-Continued

\begin{tabular}{|c|c|c|c|c|c|c|c|}
\hline \multirow{2}{*}{$\begin{array}{l}\text { Parameter } \\
\text { no. }\end{array}$} & \multirow{2}{*}{$\begin{array}{l}\text { Water-quality property or constituent } \\
\text { (unit) }\end{array}$} & \multirow{2}{*}{$\begin{array}{l}\text { No. of } \\
\text { sam- } \\
\text { ples }\end{array}$} & \multirow{2}{*}{$\begin{array}{l}\text { No. of } \\
\text { detec- } \\
\text { tions }\end{array}$} & \multirow{2}{*}{$\begin{array}{l}\text { Minimum } \\
\text { reporting } \\
\text { limit }\end{array}$} & \multicolumn{3}{|c|}{ Descriptive statistics } \\
\hline & & & & & Maximum & Minimum & Mean \\
\hline \multicolumn{8}{|c|}{ Carbon, nitrogen, and phosphorus } \\
\hline P00680 & Carbon, organic, total (mg/L as C) & 134 & 134 & 0.40 & 23 & 5.0 & 7.4 \\
\hline P00610 & Nitrogen, ammonia, total $(\mathrm{mg} / \mathrm{L}$ as $\mathrm{N})$ & 77 & 77 & .01 & 2.0 & .02 & .21 \\
\hline P00608 & Nitrogen, ammonia, dissolved $(\mathrm{mg} / \mathrm{L}$ as $\mathrm{N})$ & 57 & 57 & .02 & 3.2 & .02 & .23 \\
\hline P00625 & Nitrogen, ammonia + organic, total $(\mathrm{mg} / \mathrm{L}$ as $\mathrm{N})$ & 77 & 77 & .10 & 6.2 & .40 & 1.3 \\
\hline P00623 & Nitrogen, ammonia + organic, dissolved $(\mathrm{mg} / \mathrm{L}$ as $\mathrm{N})$ & 57 & 57 & .10 & 4.0 & .50 & 1.0 \\
\hline P00615 & Nitrogen, nitrite, total (mg/L as N) & 77 & 76 & .01 & 1.4 & $<.01$ & .10 \\
\hline P00613 & Nitrogen, nitrite, dissolved ( $\mathrm{mg} / \mathrm{L}$ as $\mathrm{N}$ ) & 57 & 57 & .01 & .96 & .02 & .09 \\
\hline P00630 & Nitrogen, nitrite + nitrate, total $(\mathrm{mg} / \mathrm{L}$ as $\mathrm{N})$ & 77 & 76 & .10 & 9.7 & $<.10$ & 4.6 \\
\hline P00631 & Nitrogen, nitrite + nitrate, dissolved $(\mathrm{mg} / \mathrm{L}$ as $\mathrm{N})$ & 57 & 57 & .06 & 7.1 & .34 & 4.1 \\
\hline P00665 & Phosphorus, total (mg/L as $\mathrm{P}$ ) & 77 & 77 & .04 & 12 & .16 & 2.2 \\
\hline P00666 & Phosphorus, dissolved (mg/L as $\mathrm{P}$ ) & 57 & 57 & .04 & 1.7 & .16 & .71 \\
\hline P00671 & Phosphorus, orthophosphate, dissolved (mg/L as $\mathrm{P}$ ) & 57 & 57 & .02 & 1.7 & .12 & .71 \\
\hline \multicolumn{8}{|c|}{ Major ions } \\
\hline P00915 & Calcium, dissolved $(\mathrm{mg} / \mathrm{L}$ as $\mathrm{Ca})$ & 114 & 114 & .01 & 75 & 9.6 & 44 \\
\hline P00925 & Magnesium, dissolved (mg/L as $\mathrm{Mg}$ ) & 114 & 114 & .01 & 16 & 1.5 & 7.9 \\
\hline P00935 & Potassium dissolved (mg/L as $\mathrm{K})$ & 109 & 109 & .11 & 9.8 & 2.3 & 5.9 \\
\hline P00930 & Sodium, dissolved (mg/L as Na) & 114 & 114 & .09 & 290 & 7.5 & 86 \\
\hline P00940 & Chloride, dissolved ( $\mathrm{mg} / \mathrm{L}$ as $\mathrm{Cl}$ ) & 109 & 109 & .01 & 180 & 7.0 & 71 \\
\hline P00950 & Fluoride, dissolved (mg/L as F) & 109 & 105 & .10 & 1.0 & $<.10$ & .40 \\
\hline P00945 & Sulfate, dissolved $\left(\mathrm{mg} / \mathrm{L}\right.$ as $\left.\mathrm{SO}_{4}\right)$ & 109 & 109 & .01 & 450 & 2.7 & 32 \\
\hline P00955 & Silica, dissolved $\left(\mathrm{mg} / \mathrm{L}\right.$ as $\left.\mathrm{SiO}_{2}\right)$ & 114 & 114 & .21 & 41 & 3.7 & 15 \\
\hline \multicolumn{8}{|c|}{ Trace elements } \\
\hline P01000 & Arsenic, dissolved ( $\mu \mathrm{g} / \mathrm{L}$ as As) & 90 & 90 & 1.9 & 21 & 2.0 & 4.0 \\
\hline P01005 & Barium, dissolved $(\mu \mathrm{g} / \mathrm{L}$ as $\mathrm{Ba})$ & 90 & 90 & .90 & 280 & 39 & 110 \\
\hline P01010 & Beryllium, dissolved $(\mu \mathrm{g} / \mathrm{L}$ as $\mathrm{Be})$ & 90 & 3 & .50 & .70 & $<.50$ & $<.50$ \\
\hline P01025 & Cadmium, dissolved $(\mu \mathrm{g} / \mathrm{L}$ as $\mathrm{Cd})$ & 90 & 11 & 1.0 & 4.0 & $<1.0$ & $<1.0$ \\
\hline P01030 & Chromium, dissolved $(\mu \mathrm{g} / \mathrm{L}$ as $\mathrm{Cr})$ & 90 & 4 & 5.0 & 9.0 & $<5.0$ & $<5.0$ \\
\hline P01035 & Cobalt, dissolved $(\mu \mathrm{g} / \mathrm{L}$ as Co $)$ & 90 & 0 & 3.0 & $<3.0$ & $<3.0$ & $<3.0$ \\
\hline P01040 & Copper, dissolved $(\mu \mathrm{g} / \mathrm{L}$ as $\mathrm{Cu})$ & 90 & 3 & 10 & 320 & $<10$ & $<10$ \\
\hline P01046 & Iron, dissolved $(\mu \mathrm{g} / \mathrm{L}$ as $\mathrm{Fe})$ & 90 & 87 & 3.0 & 190 & $<3.0$ & 21 \\
\hline
\end{tabular}


Table 6. Statistical summary of water-quality data collected at six stations during water years 1990-95-Continued

\begin{tabular}{|c|c|c|c|c|c|c|c|}
\hline \multirow{2}{*}{$\begin{array}{l}\text { Parameter } \\
\text { no. }\end{array}$} & \multirow{2}{*}{$\begin{array}{l}\text { Water-quality property or constituent } \\
\text { (unit) }\end{array}$} & \multirow{2}{*}{$\begin{array}{l}\text { No. of } \\
\text { sam- } \\
\text { ples }\end{array}$} & \multirow{2}{*}{$\begin{array}{l}\text { No. of } \\
\text { detec- } \\
\text { tions }\end{array}$} & \multirow{2}{*}{$\begin{array}{l}\text { Minimum } \\
\text { reporting } \\
\text { limit }\end{array}$} & \multicolumn{3}{|c|}{ Descriptive statistics } \\
\hline & & & & & Maximum & Minimum & Mean \\
\hline P01049 & Lead, dissolved ( $\mu \mathrm{g} / \mathrm{L}$ as $\mathrm{Pb})$ & 90 & 7 & 10 & 30 & $<10$ & $<10$ \\
\hline P01130 & Lithium, dissolved $(\mu \mathrm{g} / \mathrm{L}$ as $\mathrm{Li})$ & 90 & 82 & 4.0 & 25 & $<4.0$ & 15 \\
\hline P01056 & Manganese, dissolved ( $\mu \mathrm{g} / \mathrm{L}$ as $\mathrm{Mn})$ & 90 & 90 & 1.6 & 210 & 2.0 & 24 \\
\hline P71890 & Mercury, dissolved ( $\mu \mathrm{g} / \mathrm{L}$ as $\mathrm{Hg})$ & 90 & 2 & .1 & 2.0 & $<.1$ & $<.1$ \\
\hline P01060 & Molybdenum, dissolved ( $\mu \mathrm{g} / \mathrm{L}$ as Mo) & 90 & 24 & 10 & 20 & $<10$ & $<10$ \\
\hline P01065 & Nickel, dissolved ( $\mu \mathrm{g} / \mathrm{L}$ as Ni) & 90 & 12 & 10 & 20 & $<10$ & $<10$ \\
\hline P01145 & Selenium, dissolved ( $\mu \mathrm{g} / \mathrm{L}$ as $\mathrm{Se})$ & 90 & 20 & 1.0 & 2.0 & $<1.0$ & $<1.0$ \\
\hline P01075 & Silver, dissolved $(\mu \mathrm{g} / \mathrm{L}$ as $\mathrm{Ag})$ & 90 & 12 & 1.0 & 3.0 & $<1.0$ & $<1.0$ \\
\hline P01080 & Strontium, dissolved $(\mu \mathrm{g} / \mathrm{L}$ as $\mathrm{Sr})$ & 90 & 90 & .60 & 490 & 50 & 350 \\
\hline P01085 & Vanadium, dissolved ( $\mu \mathrm{g} / \mathrm{L}$ as $\mathrm{V})$ & 90 & 18 & 6.0 & 8.0 & $<6.0$ & $<6.0$ \\
\hline P01090 & Zinc, dissolved ( $\mu \mathrm{g} / \mathrm{L}$ as $\mathrm{Zn})$ & 90 & 90 & 1.0 & 130 & 6.0 & 17 \\
\hline \multicolumn{8}{|l|}{ Pesticides } \\
\hline P77825 & Alachlor, total, recoverable $(\mu \mathrm{g} / \mathrm{L})$ & 80 & 0 & .1 & $<.1$ & $<.1$ & $<.1$ \\
\hline P46342 & Alachlor, dissolved, recoverable $(\mu \mathrm{g} / \mathrm{L})$ & 10 & 0 & .05 & $<.05$ & $<.05$ & $<.05$ \\
\hline P82587 & Aldicarb sulfone, total, recoverable $(\mu \mathrm{g} / \mathrm{L})$ & 40 & 0 & .5 & $<.5$ & $<.5$ & $<.5$ \\
\hline P82586 & Aldicarb sulfoxide, total, recoverable ( $\mu \mathrm{g} / \mathrm{L})$ & 40 & 0 & .5 & $<.5$ & $<.5$ & $<.5$ \\
\hline P82619 & Aldicarb, total, recoverable $(\mu \mathrm{g} / \mathrm{L})$ & 74 & 0 & .5 & $<.5$ & $<.5$ & $<.5$ \\
\hline P82184 & Ametryne, total $(\mu \mathrm{g} / \mathrm{L})$ & 75 & 2 & .1 & .1 & $<.1$ & $<.1$ \\
\hline P38401 & Ametryne, dissolved, recoverable $(\mu \mathrm{g} / \mathrm{L})$ & 10 & 0 & .05 & $<.05$ & $<.05$ & $<.05$ \\
\hline P39630 & Atrazine, unfiltered, recoverable $(\mu \mathrm{g} / \mathrm{L})$ & 80 & 61 & .1 & 3.3 & $<.1$ & .1 \\
\hline P39632 & Atrazine, dissolved, recoverable $(\mu \mathrm{g} / \mathrm{L})$ & 10 & 9 & .05 & 1.20 & $<.05$ & .20 \\
\hline P30234 & Bromacil, recoverable $(\mu \mathrm{g} / \mathrm{L})$ & 65 & 11 & .2 & 1.2 & $<.2$ & $<.2$ \\
\hline P30235 & Butachlor, recoverable $(\mu \mathrm{g} / \mathrm{L})$ & 65 & 0 & .1 & $<.1$ & $<.1$ & $<.1$ \\
\hline P30236 & Butylate, recoverable $(\mu \mathrm{g} / \mathrm{L})$ & 63 & 1 & .1 & .2 & $<.1$ & $<.1$ \\
\hline P39750 & Carbaryl, unfiltered, recoverable $(\mu \mathrm{g} / \mathrm{L})$ & 89 & 0 & .5 & $<.5$ & $<.5$ & $<.5$ \\
\hline P82615 & Carbofuran, total, recoverable $(\mu \mathrm{g} / \mathrm{L})$ & 74 & 0 & .5 & $<.5$ & $<.5$ & $<.5$ \\
\hline P30245 & Carboxin, recoverable $(\mu \mathrm{g} / \mathrm{L})$ & 65 & 0 & .2 & $<.2$ & $<.2$ & $<.2$ \\
\hline P81757 & Cyanazine, total $(\mu \mathrm{g} / \mathrm{L})$ & 80 & 0 & .1 & $<.1$ & $<.1$ & $<.1$ \\
\hline
\end{tabular}


Table 6. Statistical summary of water-quality data collected at six stations during water years 1990-95-Continued

\begin{tabular}{|c|c|c|c|c|c|c|c|}
\hline \multirow{2}{*}{$\begin{array}{l}\text { Parameter } \\
\text { no. }\end{array}$} & \multirow{2}{*}{$\begin{array}{l}\text { Water-quality property or constituent } \\
\text { (unit) }\end{array}$} & \multirow{2}{*}{$\begin{array}{l}\text { No. of } \\
\text { sam- } \\
\text { ples }\end{array}$} & \multirow{2}{*}{$\begin{array}{l}\text { No. of } \\
\text { detec- } \\
\text { tions }\end{array}$} & \multirow{2}{*}{$\begin{array}{l}\text { Minimum } \\
\text { reporting } \\
\quad \text { limit }\end{array}$} & \multicolumn{3}{|c|}{ Descriptive statistics } \\
\hline & & & & & Maximum & Minimum & Mean \\
\hline P30254 & Cycloate, recoverable $(\mu \mathrm{g} / \mathrm{L})$ & 65 & 0 & 0.1 & $<0.1$ & $<0.1$ & $<0.1$ \\
\hline P75981 & Deethylatrazine, total $(\mu \mathrm{g} / \mathrm{L})$ & 60 & 9 & .2 & .4 & $<.2$ & $<.2$ \\
\hline P75980 & De-isopropylatrazine, total $(\mu \mathrm{g} / \mathrm{L})$ & 60 & 0 & .2 & $<.2$ & $<.2$ & $<.2$ \\
\hline P30264 & Hexazinone, recoverable $(\mu \mathrm{g} / \mathrm{L})$ & 65 & 3 & .2 & 1.1 & $<.2$ & $<.2$ \\
\hline P30282 & Methiocarb, recoverable $(\mu \mathrm{g} / \mathrm{L})$ & 69 & 0 & .5 & $<.5$ & $<.5$ & $<.5$ \\
\hline P39051 & Methomyl, total $(\mu \mathrm{g} / \mathrm{L})$ & 89 & 0 & .5 & $<.5$ & $<.5$ & $<.5$ \\
\hline P82612 & Metolachlor, total, recoverable $(\mu \mathrm{g} / \mathrm{L})$ & 80 & 1 & .1 & .1 & $<.1$ & $<.1$ \\
\hline P39415 & Metolachlor, dissolved $(\mu \mathrm{g} / \mathrm{L})$ & 10 & 0 & .05 & $<.05$ & $<.05$ & $<.05$ \\
\hline P82611 & Metribuzin, total, recoverable $(\mu \mathrm{g} / \mathrm{L})$ & 80 & 2 & .1 & .1 & $<.1$ & $<.1$ \\
\hline P82630 & Metribuzin, dissolved $(\mu \mathrm{g} / \mathrm{L})$ & 10 & 0 & .05 & $<.05$ & $<.05$ & $<.05$ \\
\hline P82613 & Oxyamyl, total, recoverable $(\mu \mathrm{g} / \mathrm{L})$ & 40 & 0 & .5 & $<.5$ & $<.5$ & $<.5$ \\
\hline P39056 & Prometone, total $(\mu \mathrm{g} / \mathrm{L})$ & 80 & 16 & .1 & 1.1 & $<.1$ & $<.1$ \\
\hline P39057 & Prometryne, total $(\mu \mathrm{g} / \mathrm{L})$ & 80 & 0 & .1 & $<.1$ & $<.1$ & $<.1$ \\
\hline P30295 & Propachlor, recoverable $(\mu \mathrm{g} / \mathrm{L})$ & 65 & 4 & .1 & .2 & $<.1$ & $<.1$ \\
\hline P39024 & Propazine, total $(\mu \mathrm{g} / \mathrm{L})$ & 80 & 2 & .1 & .2 & $<.1$ & $<.1$ \\
\hline P38535 & Propazine, dissolved, recoverable $(\mu \mathrm{g} / \mathrm{L})$ & 10 & 0 & .05 & $<.05$ & $<.05$ & $<.05$ \\
\hline P39052 & Propham, total $(\mu \mathrm{g} / \mathrm{L})$ & 89 & 0 & .5 & $<.5$ & $<.5$ & $<.5$ \\
\hline P30296 & Propoxur, recoverable $(\mu \mathrm{g} / \mathrm{L})$ & 69 & 1 & .5 & .7 & $<.5$ & $<.5$ \\
\hline P39055 & Simazine, total $(\mu \mathrm{g} / \mathrm{L})$ & 80 & 26 & .1 & .3 & $<.1$ & $<.1$ \\
\hline P39054 & Simetryne, total $(\mu \mathrm{g} / \mathrm{L})$ & 80 & 1 & .1 & .1 & $<.1$ & $<.1$ \\
\hline P39311 & Terbacil, recoverable $(\mu \mathrm{g} / \mathrm{L})$ & 65 & 0 & .2 & $<.2$ & $<.2$ & $<.2$ \\
\hline P39030 & Trifluralin, total, recoverable $(\mu \mathrm{g} / \mathrm{L})$ & 80 & 0 & .1 & $<.1$ & $<.1$ & $<.1$ \\
\hline P30324 & Vernolate, recoverable $(\mu \mathrm{g} / \mathrm{L})$ & 63 & 0 & .1 & $<.1$ & $<.1$ & $<.1$ \\
\hline
\end{tabular}




\section{Appendix 1-Description of Precipitation Data}




\section{Appendix 1. Description of precipitation data}

\begin{tabular}{|c|c|c|c|c|}
\hline $\begin{array}{c}\text { USGS } \\
\text { station no. }\end{array}$ & File name & Period of unit-value data & Frequency & $\begin{array}{c}\text { Maximum } \\
\text { precipitation } \\
\text { intensity } \\
\text { (inches/hour) }\end{array}$ \\
\hline \multirow[t]{21}{*}{08073600} & p73600_0191.rdb & 01-09-91@0200 to 01-18-91@ 1345 & 15-minute & 1.58 \\
\hline & p73600_0291.rdb & 02-04-91@0800 to 02-04-91@ 1245 & 15-minute & 1.37 \\
\hline & p73600_0491a.rdb & 04-04-91@ @ 1100 to 04-06-91@0500 & 15-minute & .46 \\
\hline & p73600_0491b.rdb & 04-14-91@0700 to 04-17-91 @ 2215 & 15-minute & 2.52 \\
\hline & p73600_1291.rdb & 12-21-91@0600 to 12-26-91@2245 & 15-minute & .78 \\
\hline & p73600_0392.rdb & 03-04-92@0100 to 03-06-92 @0130 & 15-minute & 2.84 \\
\hline & p73600_0492.rdb & 04-17-92@1500 to 04-19-92 @ 2315 & 15-minute & 2.68 \\
\hline & p73600_0592.rdb & 05-16-92@0200 to 05-17-92@0945 & 15-minute & 1.58 \\
\hline & p73600_0892.rdb & 08-02-92@1700 to 08-03-92 @0215 & 15-minute & 4.10 \\
\hline & p73600_0293.rdb & 02-25-93@0900 to 03-02-93@0315 & 15-minute & 1.98 \\
\hline & p73600_0393.rdb & 03-19-93@2200 to 03-22-93@2315 & 15-minute & 1.07 \\
\hline & p73600_0493.rdb & 04-03-93@1200 to 04-07-93@ 1515 & 15-minute & 2.46 \\
\hline & p73600_0693.rdb & 06-17-93@1300 to 06-20-93@1530 & 15-minute & 2.20 \\
\hline & p73600_1093.rdb & 10-12-93@1200 to 10-21-93@0530 & 15-minute & 1.54 \\
\hline & p73600_1193.rdb & 11-13-93@2100 to 11-16-93@2015 & 15-minute & .82 \\
\hline & p73600_0694.rdb & 06-17-94@1600 to 06-24-94@ 1830 & 15-minute & 2.52 \\
\hline & p73600_0894.rdb & 08-17-94@1500 to 08-27-94@1500 & 15-minute & 4.15 \\
\hline & p73600_1094.rdb & 10-15-94@0700 to 10-18-94@2100 & 15-minute & 2.14 \\
\hline & p73600_0195.rdb & 01-12-95@2000 to 01-26-95 @ 2030 & 15-minute & 2.67 \\
\hline & p73600_0395.rdb & 03-13-95@0200 to 03-13-95 @0845 & 15-minute & 1.96 \\
\hline & p73600_0495.rdb & 04-04-95@0500 to 04-05-95@ @ 1700 & 15-minute & 2.37 \\
\hline \multirow[t]{19}{*}{08074020} & p74020_1089.rdb & 10-29-89@1300 to 10-30-89 @ 1630 & 15-minute & 8.99 \\
\hline & p74020_0290.rdb & 02-21-90@0700 to 02-21-90@ @ 1445 & 15-minute & .64 \\
\hline & p74020_0490.rdb & 04-26-90@1300 to 04-27-90@2345 & 15-minute & 3.86 \\
\hline & p74020_0191a.rdb & 01-01-91@1000 to 01-10-91@0945 & 15-minute & 1.47 \\
\hline & p74020_0191b.rdb & 01-18-91@0600 to 01-19-91@ @ 1400 & 15-minute & 1.89 \\
\hline & p74020_0291.rdb & 02-04-91@0800 to 02-04-91 @ 1800 & 15-minute & .90 \\
\hline & p74020_0491a.rdb & 04-04-91@0300 to 04-06-91@ @ 1600 & 15-minute & 1.72 \\
\hline & p74020_0491b.rdb & 04-14-91@0700 to 04-14-91@ @1430 & 15-minute & 2.03 \\
\hline & p74020_0691.rdb & 06-14-91@1700 to 06-18-91@2100 & 15-minute & 5.75 \\
\hline & p74020_1191.rdb & 11-17-91@0100 to 11-17-91@ 1330 & 15-minute & 2.36 \\
\hline & p74020_1291.rdb & 12-21-91@0000 to 12-26-91@0545 & 15-minute & 1.10 \\
\hline & p74020_0392.rdb & 03-04-92@0100 to 03-06-92 @0100 & 15-minute & 3.21 \\
\hline & p74020_0492.rdb & 04-17-92@1500 to 04-17-92 @ 2030 & 15-minute & 1.64 \\
\hline & p74020_0592.rdb & 05-16-92@0300 to 05-18-92@ 1830 & 15-minute & 1.93 \\
\hline & p74020_0692.rdb & 06-01-92@1500 to 06-02-92 @0900 & 15-minute & 3.46 \\
\hline & p74020_0293.rdb & 02-28-93@2000 to 03-02-93@0200 & 15-minute & 2.28 \\
\hline & p74020_0393.rdb & 03-22-93@0400 to 03-22-93@ 2315 & 15-minute & 2.99 \\
\hline & p74020_0493a.rdb & 04-03-93@1200 to 04-04-93@0630 & 15-minute & 2.54 \\
\hline & p74020_0493b.rdb & 04-07-93@1300 to 04-07-93 @ 1515 & 15-minute & 3.15 \\
\hline
\end{tabular}


Appendix 1. Description of precipitation data-Continued

\begin{tabular}{|c|c|c|c|c|}
\hline $\begin{array}{c}\text { USGS } \\
\text { station no. }\end{array}$ & File name & Period of unit-value data & Frequency & $\begin{array}{c}\text { Maximum } \\
\text { precipitation } \\
\text { intensity } \\
\text { (inches/hour) }\end{array}$ \\
\hline & p74020_0693.rdb & 06-17-93@1400 to 06-22-93@1600 & 15-minute & 1.28 \\
\hline & p74020_1193.rdb & 11-13-93@2300 to 11-16-93@1315 & 15-minute & 1.00 \\
\hline & p74020_0294.rdb & 02-09-94@1200 to 02-10-94 @ 1715 & 15-minute & .60 \\
\hline & p74020_0594.rdb & 05-15-94@0700 to 05-17-94@1600 & 15-minute & 1.49 \\
\hline & p74020_0694.rdb & 06-20-94@1700 to 06-24-94 @1815 & 15-minute & 3.23 \\
\hline & p74020_0894a.rdb & 08-04-94@1100 to 08-05-94 @ 1515 & 15-minute & 3.94 \\
\hline & p74020_0894b.rdb & 08-15-94@1700 to 08-23-94@1200 & 15-minute & 1.28 \\
\hline & p74020_1094.rdb & 10-15-94@0600 to 10-18-94 @2015 & 15-minute & 1.43 \\
\hline & p74020_0195.rdb & 01-25-95@2300 to 01-27-95@0200 & 15-minute & .58 \\
\hline & p74020_0395.rdb & 03-13-95@0700 to 03-15-95 @1845 & 15-minute & 2.68 \\
\hline & p74020_0495.rdb & 04-04-95@0600 to 04-05-95@1745 & 15-minute & 1.16 \\
\hline \multirow[t]{27}{*}{08074150} & p74150_1089.rdb & 10-29-89@1000 to 10-30-89 @1645 & 15-minute & 6.39 \\
\hline & p74150_0290.rdb & 02-21-90@0800 to 02-21-90 @ 1500 & 15-minute & .71 \\
\hline & p74150_0490.rdb & 04-26-90@1300 to 04-28-90 @0045 & 15-minute & 1.46 \\
\hline & p74150_0890.rdb & 08-14-90@1900 to 08-15-90@1945 & 15-minute & 1.89 \\
\hline & p74150_0291.rdb & 02-04-91@0800 to 02-04-91@1900 & 15-minute & .62 \\
\hline & p74150_0491a.rdb & 04-04-91@0900 to 04-06-91@0715 & 15-minute & .58 \\
\hline & p74150_0491b.rdb & 04-14-91@1700 to 04-19-91@1645 & 15-minute & 1.79 \\
\hline & p74150_0691.rdb & 06-14-91@1600 to 06-18-91@2000 & 15-minute & 5.28 \\
\hline & p74150_1191.rdb & 11-17-91@1100 to 11-20-91@0600 & 15-minute & .42 \\
\hline & p74150_1291.rdb & 12-21-91@0100 to 12-27-91@0330 & 15-minute & .58 \\
\hline & p74150_0392.rdb & 03-04-92@0100 to 03-06-92@0800 & 15-minute & 2.47 \\
\hline & p74150_0492.rdb & 04-17-92@1500 to 04-20-92 @1815 & 15-minute & 3.54 \\
\hline & p74150_0592.rdb & 05-16-92@0300 to 05-20-92 @2015 & 15-minute & 2.07 \\
\hline & p74150_0692.rdb & 05-31-92@1300 to 06-02-92 @0815 & 15-minute & 3.04 \\
\hline & p74150_0293.rdb & 02-28-93@2000 to 03-02-93@0215 & 15-minute & 1.87 \\
\hline & p74150_0393.rdb & 03-22-93@1600 to 03-22-93@2100 & 15-minute & 1.08 \\
\hline & p74150_0493.rdb & 04-03-93@1200 to 04-07-93@1615 & 15-minute & 3.38 \\
\hline & p74150_0693.rdb & 06-17-93@1400 to 06-21-93@2130 & 15-minute & 1.75 \\
\hline & p74150_1193.rdb & 11-14-93@0900 to 11-16-93@1815 & 15-minute & 1.02 \\
\hline & p74150_0294.rdb & 02-20-94@1600 to 02-22-94@1900 & 15-minute & 3.00 \\
\hline & p74150_0494.rdb & 04-30-94@0200 to 05-03-94@0215 & 15-minute & 2.62 \\
\hline & p74150_0694.rdb & 06-20-94@1600 to 06-24-94 @1815 & 15-minute & 2.05 \\
\hline & p74150_0894.rdb & 08-16-94@0600 to 08-26-94@ @ 1730 & 15-minute & 3.46 \\
\hline & p74150_1094.rdb & 10-15-94@1000 to 10-18-94@2015 & 15-minute & 2.36 \\
\hline & p74150_0195.rdb & 01-17-95@2100 to 01-27-95@0215 & 15-minute & 2.33 \\
\hline & p74150_0395.rdb & 03-13-95@0400 to 03-15-95@1930 & 15-minute & 3.04 \\
\hline & p74150_0495.rdb & 04-04-95@0500 to 04-10-95@2115 & 15-minute & 3.62 \\
\hline \multirow[t]{3}{*}{08074250} & p74250_1089.rdb & 10-29-89@0900 to 10-30-89 @1630 & 15-minute & 1.97 \\
\hline & p74250_0290.rdb & 02-21-90@0700 to 02-21-90@1315 & 15-minute & .67 \\
\hline & p74250_0490.rdb & 04-26-90@1300 to 04-28-90 @1300 & 15-minute & 2.36 \\
\hline
\end{tabular}


Appendix 1. Description of precipitation data-Continued

\begin{tabular}{|c|c|c|c|c|}
\hline $\begin{array}{c}\text { USGS } \\
\text { station no. }\end{array}$ & File name & Period of unit-value data & Frequency & $\begin{array}{l}\text { Maximum } \\
\text { precipitation } \\
\text { intensity } \\
\text { (inches/hour) }\end{array}$ \\
\hline & p74250_0890.rdb & 08-14-90 @ 1900 to 08-15-90 @ 1815 & 15-minute & 3.02 \\
\hline & p74250_0191.rdb & 01-14-91 @ 1400 to 01-18-91 @ 1415 & 15-minute & 1.80 \\
\hline & p74250_0291.rdb & 02-04-91 @0800 to 02-05-91 @ 1400 & 15-minute & 1.04 \\
\hline & p74250_0491.rdb & 04-10-91@1300 to 04-17-91@2215 & 15-minute & 3.35 \\
\hline & p74250_0691.rdb & 06-14-91@ 1700 to 06-17-91@2000 & 15-minute & 2.99 \\
\hline & p74250_1191.rdb & 11-17-91@0100 to 11-17-91@ 1345 & 15-minute & 2.30 \\
\hline & p74250_1291.rdb & 12-21-91@0100 to 12-27-91@0830 & 15-minute & .50 \\
\hline & p74250_0392.rdb & 03-04-92@0100 to 03-06-92@0315 & 15-minute & 2.15 \\
\hline & p74250_0492.rdb & 04-17-92@1500 to 04-20-92@0730 & 15-minute & 2.04 \\
\hline & p74250_0592.rdb & 05-15-92@ @ 1500 to 05-17-92 @ 1645 & 15-minute & .38 \\
\hline & p74250_0293.rdb & 02-28-93@2100 to 03-02-93 @ 0230 & 15-minute & 1.11 \\
\hline & p74250_0393.rdb & 03-19-93@2200 to 03-22-93@2245 & 15-minute & 1.34 \\
\hline & p74250_0493.rdb & 04-03-93@ 1200 to 04-07-93@ 1530 & 15-minute & 3.65 \\
\hline & p74250_0693.rdb & 06-17-93@1500 to 06-22-93@ 1545 & 15-minute & 3.74 \\
\hline & p74250_1193.rdb & 11-14-93@0800 to 11-16-93@ 1330 & 15-minute & 1.45 \\
\hline & p74250_0294.rdb & 02-20-94@ @ 1700 to 02-23-94@0700 & 15-minute & .92 \\
\hline & p74250_0494.rdb & 04-30-94@0800 to 05-03-94@0200 & 15-minute & 2.30 \\
\hline & p74250_0694.rdb & 06-17-94@ @ 1800 to 06-24-94@ 1815 & 15-minute & 2.76 \\
\hline & p74250_0894.rdb & 08-17-94@ @ 1700 to 08-23-94@ 1215 & 15-minute & 4.10 \\
\hline & p74250_1094.rdb & 10-15-94@0700 to 10-19-94@0645 & 15-minute & 1.86 \\
\hline & p74250_0195.rdb & 01-12-95@2000 to 01-27-95@0700 & 15-minute & 2.40 \\
\hline & p74250_0395.rdb & 03-13-95@0400 to 03-16-95@0015 & 15-minute & 2.32 \\
\hline & p74250_0495.rdb & 04-04-95 @0200 to 04-05-95 @ 1815 & 15-minute & 1.90 \\
\hline \multirow[t]{18}{*}{08074500} & p74500_1089.rdb & 10-29-89@1400 to 11-01-89@2030 & 15-minute & 3.80 \\
\hline & p74500_0290.rdb & 02-21-90@0700 to 02-21-90 @ 1445 & 15-minute & .45 \\
\hline & p74500_0490.rdb & 04-26-90@1300 to 04-28-90@0745 & 15-minute & 2.30 \\
\hline & p74500_0191.rdb & 01-14-91 @ 1400 to 01-18-91 @ 1415 & 15-minute & 1.48 \\
\hline & p74500_0291.rdb & 02-04-91 @0800 to 02-04-91 @ 1945 & 15-minute & 7.44 \\
\hline & p74500_0491.rdb & 04-10-91 @ 1400 to 04-19-91@0630 & 15-minute & 4.69 \\
\hline & p74500_0691.rdb & 06-14-91 @ 1800 to 06-17-91@ @ 1800 & 15-minute & 4.07 \\
\hline & p74500_1191.rdb & 11-17-91@0300 to 11-19-91@ 1845 & 15-minute & 8.34 \\
\hline & p74500_1291.rdb & 12-21-91@0600 to 12-30-91@ @ 1500 & 15-minute & 4.71 \\
\hline & p74500_0392.rdb & 03-04-92@0100 to 03-06-92 @0515 & 15-minute & 2.05 \\
\hline & p74500_0592.rdb & 05-16-92@0500 to 05-20-92 @ 1615 & 15-minute & 1.58 \\
\hline & p74500_0293.rdb & 02-25-93@0900 to 03-02-93 @ 1345 & 15-minute & 2.41 \\
\hline & p74500_0393.rdb & 03-22-93@ 1500 to 03-22-93 @ 2115 & 15-minute & 4.86 \\
\hline & p74500_0493.rdb & 04-07-93@ 1200 to 04-07-93@ 1545 & 15-minute & 1.50 \\
\hline & p74500_0693.rdb & 06-17-93 @ 1400 to 06-23-93@ 1615 & 15-minute & 1.58 \\
\hline & p74500_1193.rdb & 11-14-93@0600 to 11-16-93@ 1315 & 15-minute & 2.10 \\
\hline & p74500_0294.rdb & 02-20-94@1900 to 02-22-94@ @ 1415 & 15 -minute & .26 \\
\hline & p74500_0694.rdb & $06-20-94 @ 1700$ to $06-25-94 @ 2145$ & 15 -minute & 2.20 \\
\hline
\end{tabular}


Appendix 1. Description of precipitation data-Continued

\begin{tabular}{|c|c|c|c|c|}
\hline $\begin{array}{c}\text { USGS } \\
\text { station no. }\end{array}$ & File name & Period of unit-value data & Frequency & $\begin{array}{c}\text { Maximum } \\
\text { precipitation } \\
\text { intensity } \\
\text { (inches/hour) }\end{array}$ \\
\hline & p74500_0894.rdb & 08-15-94@1800 to 08-22-94@1730 & 15-minute & 2.70 \\
\hline & p74500_1094.rdb & 10-15-94@1000 to 10-18-94 @2100 & 15-minute & 2.36 \\
\hline & p74500_0195.rdb & 01-12-95@2000 to 01-27-95@0145 & 15-minute & 2.52 \\
\hline & p74500_0395.rdb & 03-13-95@0400 to 03-15-95@1930 & 15-minute & 1.94 \\
\hline & p74500_0495.rdb & 04-04-95@0900 to 04-05-95@1730 & 15-minute & 3.31 \\
\hline \multirow[t]{11}{*}{08074540} & p74540_0191.rdb & 01-09-91@0200 to 01-10-91@0900 & 15-minute & 2.24 \\
\hline & p74540_0491a.rdb & 04-04-91@1000 to 04-05-91@2100 & 15-minute & .58 \\
\hline & p74540_0491b.rdb & 04-11-91@1800 to 04-17-91 @2230 & 15-minute & 1.74 \\
\hline & p74540_0691.rdb & 06-15-91@0500 to 06-17-91@1315 & 15-minute & 1.43 \\
\hline & p74540_1191.rdb & 11-17-91@0600 to 11-19-91@1515 & 15-minute & 3.46 \\
\hline & p74540_0392.rdb & 03-04-92 @0100 to 03-06-92 @ 1215 & 15-minute & 1.82 \\
\hline & p74540_0393.rdb & 03-22-93@1500 to 03-23-93@1915 & 15-minute & 1.75 \\
\hline & p74540_0693.rdb & 06-17-93@1400 to 06-27-93@2215 & 15-minute & 1.48 \\
\hline & p74540_1093.rdb & 10-20-93@0600 to 10-20-93@1845 & 15-minute & 2.54 \\
\hline & p74540_0894.rdb & 08-15-94@1800 to 08-17-94@1830 & 15-minute & 2.68 \\
\hline & p74540_0495.rdb & 04-04-95@0700 to 04-05-95@1945 & 15-minute & 1.42 \\
\hline \multirow[t]{25}{*}{08074760} & p74760_0191a.rdb & 01-09-91@1100 to 01-10-91@0945 & 15-minute & 1.42 \\
\hline & p74760_0191b.rdb & 01-14-91@1300 to 01-15-91@0000 & 15-minute & 2.41 \\
\hline & p74760_0291.rdb & 02-04-91@0800 to 02-04-91@1800 & 15-minute & 1.64 \\
\hline & p74760_0491a.rdb & 04-04-91@1000 to 04-05-91@ @ 1715 & 15-minute & 1.30 \\
\hline & p74760_0491b.rdb & 04-14-91@0700 to 04-15-91@1345 & 15-minute & 3.02 \\
\hline & p74760_0292.rdb & 02-22-92@0400 to 02-22-92 @1315 & 15-minute & 4.19 \\
\hline & p74760_0392.rdb & 03-04-92@0100 to 03-06-92 @0145 & 15-minute & 3.10 \\
\hline & p74760_0492.rdb & 04-17-92@1400 to 04-19-92 @ 2230 & 15-minute & 2.05 \\
\hline & p74760_0592.rdb & 05-27-92@0700 to 06-02-92 @0730 & 15-minute & 3.31 \\
\hline & p74760_1192.rdb & 11-19-92@1300 to 11-21-92@1645 & 15-minute & 1.97 \\
\hline & p74760_0293.rdb & 02-25-93@0900 to 03-02-93@0200 & 15-minute & 2.36 \\
\hline & p74760_0393a.rdb & 02-28-93@2100 to 03-02-93@0200 & 15-minute & 1.34 \\
\hline & p74760_0393b.rdb & 03-22-93@1900 to 03-22-93@2130 & 15-minute & 2.52 \\
\hline & p74760_0493.rdb & 04-03-93@1200 to 04-07-93@1745 & 15-minute & 3.40 \\
\hline & p74760_0693.rdb & 06-15-93@1500 to 06-27-93@1815 & 15-minute & 2.52 \\
\hline & p74760_1093.rdb & 10-20-93@1100 to 10-21-93@0245 & 15-minute & 2.52 \\
\hline & p74760_1193.rdb & 11-13-93@1400 to 11-17-93@0315 & 15-minute & .98 \\
\hline & p74760_0294.rdb & 02-09-94@0900 to 02-10-94 @1645 & 15-minute & .62 \\
\hline & p74760_0594.rdb & 05-13-94@1500 to 05-17-94@2045 & 15-minute & 1.12 \\
\hline & p74760_0694a.rdb & 06-10-94@1000 to 06-12-94@1500 & 15-minute & 3.46 \\
\hline & p74760_0694b.rdb & 06-17-94@1500 to 06-24-94 @1545 & 15-minute & 2.80 \\
\hline & p74760_0994.rdb & 09-08-94@1900 to 09-09-94 @1130 & 15-minute & 4.95 \\
\hline & p74760_1094.rdb & 10-15-94@0300 to 10-18-94@2045 & 15-minute & 3.36 \\
\hline & p74760_0195a.rdb & 01-12-95@2000 to 01-12-95 @2315 & 15-minute & 3.07 \\
\hline & p74760_0195b.rdb & 01-18-95@0700 to 01-26-95@2045 & 15-minute & 2.52 \\
\hline
\end{tabular}


Appendix 1. Description of precipitation data-Continued

\begin{tabular}{|c|c|c|c|c|}
\hline $\begin{array}{c}\text { USGS } \\
\text { station no. }\end{array}$ & File name & Period of unit-value data & Frequency & $\begin{array}{c}\text { Maximum } \\
\text { precipitation } \\
\text { intensity } \\
\text { (inches/hour) }\end{array}$ \\
\hline & p74760_0195c.rdb & 01-25-95 @2100 to 01-26-95 @ 2045 & 15-minute & 1.30 \\
\hline & p74760_0395.rdb & 03-12-95@0100 to 03-16-95@0315 & 15-minute & 1.89 \\
\hline & p74760_0495.rdb & 04-04-95 @0500 to 04-05-95 @ 1730 & 15-minute & 1.94 \\
\hline & p74760_0595.rdb & 05-27-95@0800 to 06-01-95 @ 1015 & 15-minute & 2.92 \\
\hline \multirow[t]{30}{*}{08074780} & p74780_0191a.rdb & 01-09-91 @ 1200 to 01-10-91 @ 1015 & 15-minute & 1.58 \\
\hline & p74780_0191b.rdb & 01-14-91@1300 to 01-15-91@0000 & 15-minute & 2.20 \\
\hline & p74780_0291.rdb & |02-04-91@0700 to 02-04-91 @ 1815 & 15-minute & 1.80 \\
\hline & p74780_0491a.rdb & 04-04-91@0800 to 04-17-91 @ 2215 & 15-minute & 2.08 \\
\hline & p74780_0491b.rdb & 04-14-91@0800 to 04-17-91@2215 & 15-minute & 2.08 \\
\hline & p74780_0691.rdb & 06-15-91@0800 to 06-16-91@ 1930 & 15-minute & 3.15 \\
\hline & p74780_0292.rdb & 02-22-92@0400 to 02-22-92 @ 0815 & 15-minute & 4.75 \\
\hline & p74780_0392.rdb & 03-04-92@0100 to 03-06-92@ @ 0100 & 15-minute & 1.99 \\
\hline & p74780_0492.rdb & 04-17-92 @ 1400 to 04-19-92 @ 2200 & 15-minute & 2.12 \\
\hline & p74780_0592.rdb & 05-27-92 @0500 to 06-02-92 @ 1145 & 15-minute & 3.15 \\
\hline & p74780_1192.rdb & 11-19-92@ 1700 to 11-21-92@ 1500 & 15-minute & 2.14 \\
\hline & p74780_0293.rdb & 02-25-93@ 1000 to 03-02-93 @ 0200 & 15-minute & 2.68 \\
\hline & p74780_0393a.rdb & 02-28-93@2000 to 03-02-93@0200 & 15-minute & 1.96 \\
\hline & p74780_0393b.rdb & 03-19-93@2200 to 03-23-93@0345 & 15-minute & 1.20 \\
\hline & p74780_0493.rdb & 04-03-93@ 1600 to 04-04-93@0600 & 15-minute & 2.36 \\
\hline & p74780_0693.rdb & 06-17-93@ 1800 to 06-21-93@ 1200 & 15-minute & 1.83 \\
\hline & p74780_1093.rdb & 10-20-93@1100 to 10-21-93@0300 & 15-minute & 2.83 \\
\hline & p74780_1193.rdb & 11-16-93@0100 to 11-16-93@ 1245 & 15-minute & 1.02 \\
\hline & p74780_0594.rdb & 05-13-94@ @ 1500 to 05-18-94@ 1245 & 15-minute & 1.58 \\
\hline & p74780_0694a.rdb & 06-10-94@ @1500 to 06-10-94@ 1915 & 15-minute & 3.20 \\
\hline & p74780_0694b.rdb & 06-12-94@ @ 1000 to 06-24-94@ 1700 & 15-minute & 2.90 \\
\hline & p74780_0994a.rdb & 09-01-94 @0900 to 09-03-94 @ 1845 & 15-minute & 2.52 \\
\hline & p74780_0994b.rdb & 09-08-94 @ 1900 to 09-10-94 @ 1445 & 15-minute & 1.89 \\
\hline & p74780_1094.rdb & 10-15-94@0500 to 10-19-94@0300 & 15-minute & 3.99 \\
\hline & p74780_0195a.rdb & 01-12-95 @2000 to 01-12-95 @ 2300 & 15-minute & 3.53 \\
\hline & p74780_0195b.rdb & 01-18-95 @0600 to 01-27-95 @ 1445 & 15-minute & 1.54 \\
\hline & p74780_0195c.rdb & 01-25-95@2000 to 01-27-95 @ 1445 & 15-minute & .60 \\
\hline & p74780_0395.rdb & 03-12-95 @ 1900 to 03-14-95 @ 1645 & 15-minute & 1.78 \\
\hline & p74780_0495.rdb & 04-04-95 @0500 to 04-05-95@ 1730 & 15-minute & 1.80 \\
\hline & p74780_0595.rdb & 05-27-95@0800 to 06-01-95@0315 & 15-minute & 2.03 \\
\hline \multirow[t]{7}{*}{08074800} & p74800_0191a.rdb & 01-09-91 @ 1200 to 01-10-91@0945 & 15-minute & 2.30 \\
\hline & p74800_0191b.rdb & 01-14-91@1300 to 01-15-91@0000 & 15-minute & 1.67 \\
\hline & p74800_0291.rdb & 02-04-91@0800 to 02-04-91 @ 1815 & 15-minute & 1.99 \\
\hline & p74800_0491a.rdb & 04-04-91 @0800 to 04-05-91 @ 1715 & 15-minute & 1.50 \\
\hline & p74800_0491b.rdb & 04-14-91@0700 to 04-17-91 @ 2215 & 15-minute & 1.75 \\
\hline & p74800_0691.rdb & 06-15-91@0600 to 06-18-91@2315 & 15-minute & 3.60 \\
\hline & p74800_0292.rdb & 02-22-92 @0400 to 02-24-92 @ 2345 & 15-minute & 1.27 \\
\hline
\end{tabular}


Appendix 1. Description of precipitation data-Continued

\begin{tabular}{|c|c|c|c|c|}
\hline $\begin{array}{c}\text { USGS } \\
\text { station no. }\end{array}$ & File name & Period of unit-value data & Frequency & $\begin{array}{c}\text { Maximum } \\
\text { precipitation } \\
\text { intensity } \\
\text { (inches/hour) }\end{array}$ \\
\hline & p74800_0392.rdb & 03-04-92@0100 to 03-05-92 @0100 & 15-minute & 0.60 \\
\hline & p74800_0492.rdb & 04-17-92@1400 to 04-20-92 @2315 & 15-minute & 2.16 \\
\hline & p74800_0592.rdb & 05-26-92@2100 to 06-03-92 @0315 & 15-minute & 2.56 \\
\hline & p74800_1192.rdb & 11-19-92@1400 to 11-22-92 @ 1500 & 15-minute & 2.31 \\
\hline & p74800_0293.rdb & 02-25-93@1000 to 03-02-93@0200 & 15-minute & 2.21 \\
\hline & p74800_0393.rdb & 03-19-93@0100 to 03-23-93@0200 & 15-minute & .56 \\
\hline & p74800_0493.rdb & 04-03-93@1600 to 04-08-93@0915 & 15-minute & 1.86 \\
\hline & p74800_0693.rdb & 06-17-93@1600 to 06-23-93@1530 & 15-minute & 1.49 \\
\hline & p74800_1093.rdb & $10-20-93 @ 1300$ to $10-20-93 @ 1630$ & 15-minute & 2.33 \\
\hline & p74800_1193.rdb & 11-13-93@1400 to 11-16-93@1245 & 15-minute & 2.44 \\
\hline & p74800_0594.rdb & 05-13-94@1800 to 05-18-94@0115 & 15-minute & 2.24 \\
\hline & p74800_0694a.rdb & 06-10-94@1500 to 06-12-94@2030 & 15-minute & 2.05 \\
\hline & p74800_0694b.rdb & 06-16-94@1600 to 06-24-94 @1745 & 15-minute & 3.27 \\
\hline & p74800_0994a.rdb & 09-01-94@1600 to 09-04-94 @0515 & 15-minute & .10 \\
\hline & p74800_0994b.rdb & 09-09-94@0900 to 09-10-94@1300 & 15-minute & 2.42 \\
\hline & p74800_1094.rdb & 10-15-94@0900 to 10-18-94@2100 & 15-minute & 5.00 \\
\hline & p74800_0195a.rdb & 01-12-95@2000 to 01-12-95@2330 & 15-minute & 3.28 \\
\hline & p74800_0195b.rdb & 01-18-95@0500 to 01-27-95@0745 & 15-minute & 1.38 \\
\hline & p74800_0195c.rdb & 01-22-95@1400 to 01-27-95@0745 & 15-minute & .54 \\
\hline & p74800_0395.rdb & 03-12-95@1200 to 03-15-95@1945 & 15-minute & 2.26 \\
\hline & p74800_0495.rdb & 04-04-95@0500 to 04-10-95@2100 & 15-minute & 1.06 \\
\hline & p74800_0595.rdb & 05-27-95@0800 to 05-31-95@0300 & 15-minute & 2.52 \\
\hline \multirow[t]{19}{*}{08074810} & p74810_0191a.rdb & 01-09-91@1200 to 01-12-91 @1915 & 15-minute & 2.27 \\
\hline & p74810_0191b.rdb & 01-14-91@1300 to 01-15-91 @ 1815 & 15-minute & 1.80 \\
\hline & p74810_0291.rdb & 02-04-91@0800 to 02-04-91@1800 & 15-minute & 1.12 \\
\hline & p74810_0491a.rdb & 04-04-91@0200 to 04-06-91@0130 & 15-minute & 1.10 \\
\hline & p74810_0491b.rdb & 04-14-91@0500 to 04-18-91@0715 & 15-minute & 2.86 \\
\hline & p74810_0292.rdb & 02-22-92@0400 to 02-24-92 @1945 & 15-minute & 3.59 \\
\hline & p74810_0392.rdb & 03-04-92@0100 to 03-04-92 @ 1600 & 15-minute & 3.03 \\
\hline & p74810_0492.rdb & 04-17-92@1400 to 04-20-92 @0615 & 15-minute & 2.37 \\
\hline & p74810_0592.rdb & 05-26-92@2100 to 06-02-92 @0715 & 15-minute & 1.49 \\
\hline & p74810_0293.rdb & 02-25-93@1000 to 03-02-93@2030 & 15-minute & 3.20 \\
\hline & p74810_0393.rdb & 03-22-93@1900 to 03-22-93@2330 & 15-minute & .98 \\
\hline & p74810_0493.rdb & 04-03-93@1600 to 04-07-93@2330 & 15-minute & 2.12 \\
\hline & p74810_0693.rdb & 06-17-93@1300 to 06-28-93@1245 & 15-minute & 2.28 \\
\hline & p74810_1093.rdb & 10-20-93@1200 to 10-21-93@0330 & 15-minute & 2.89 \\
\hline & p74810_1193.rdb & 11-14-93@0600 to 11-16-93@1300 & 15-minute & 3.17 \\
\hline & p74810_0594.rdb & 05-13-94@2100 to 05-18-94 @0715 & 15-minute & 2.99 \\
\hline & p74810_0694a.rdb & 06-10-94@1600 to 06-12-94 @2315 & 15-minute & 1.22 \\
\hline & p74810_0694b.rdb & 06-18-94@1600 to 06-23-94@1500 & 15-minute & 1.93 \\
\hline & p74810_0994.rdb & 09-08-94@2000 to 09-10-94@1345 & 15-minute & 1.70 \\
\hline
\end{tabular}


Appendix 1. Description of precipitation data-Continued

\begin{tabular}{|c|c|c|c|c|}
\hline $\begin{array}{c}\text { USGS } \\
\text { station no. }\end{array}$ & File name & Period of unit-value data & Frequency & $\begin{array}{c}\text { Maximum } \\
\text { precipitation } \\
\text { intensity } \\
\text { (inches/hour) }\end{array}$ \\
\hline & p74810_1094.rdb & 10-15-94@0400 to 10-18-94@2000 & 15-minute & 4.33 \\
\hline & p74810_0195a.rdb & 01-12-95 @2000 to 01-12-95 @ 2345 & 15-minute & 3.23 \\
\hline & p74810_0195b.rdb & 01-18-95@0600 to 01-27-95@0145 & 15-minute & 1.35 \\
\hline & p74810_0395.rdb & 03-13-95@0600 to 03-15-95 @ 1945 & 15-minute & 2.28 \\
\hline & p74810_0495.rdb & 04-04-95 @0600 to 04-05-95 @ 1830 & 15-minute & .83 \\
\hline & p74810_0595.rdb & 05-27-95@0800 to 06-01-95@0345 & 15-minute & 2.15 \\
\hline \multirow[t]{23}{*}{08075000} & p75000_0191.rdb & 01-14-91@1300 to 01-18-91 @ 1415 & 15-minute & 3.24 \\
\hline & p75000_0291.rdb & 02-04-91 @0800 to 02-06-91 @ 1100 & 15-minute & 1.14 \\
\hline & p75000_0491a.rdb & 04-04-91@0200 to 04-11-91@ @ 1900 & 15-minute & 2.47 \\
\hline & p75000_0491b.rdb & 04-14-91@0900 to 04-19-91 @ 1645 & 15-minute & 3.62 \\
\hline & p75000_0292.rdb & 02-22-92 @0400 to 02-24-92 @ 2045 & 15-minute & 3.97 \\
\hline & p75000_0392.rdb & 03-04-92@0100 to 03-06-92@1330 & 15-minute & 2.15 \\
\hline & p75000_0492.rdb & 04-17-92@1600 to 04-20-92@0745 & 15-minute & 2.53 \\
\hline & p75000_0592.rdb & 05-26-92 @2200 to 06-02-92 @ 1230 & 15-minute & 2.02 \\
\hline & p75000_1192.rdb & 11-19-92@1400 to 11-21-92@1545 & 15-minute & 2.70 \\
\hline & p75000_0293.rdb & 02-25-93 @ 1000 to 03-02-93@ 1000 & 15-minute & 2.50 \\
\hline & p75000_0393.rdb & 03-19-93@2300 to 03-23-93@0600 & 15-minute & 1.60 \\
\hline & p75000_0693.rdb & 06-17-93@1300 to 06-27-93@1830 & 15-minute & 2.84 \\
\hline & p75000_1093.rdb & 10-20-93@ @ 1100 to 10-21-93@0500 & 15-minute & 3.94 \\
\hline & p75000_1193.rdb & 11-13-93@2100 to 11-16-93@ 1345 & 15-minute & 4.10 \\
\hline & p75000_0594.rdb & 05-13-94@1800 to 05-18-94@0630 & 15-minute & .66 \\
\hline & p75000_0694.rdb & 06-10-94@1500 to 06-25-94@ 1815 & 15-minute & 2.20 \\
\hline & p75000_0994.rdb & 09-01-94 @0800 to 09-09-94@ @ 1300 & 15-minute & 1.10 \\
\hline & p75000_1094.rdb & 10-15-94@1200 to 10-18-94@ @ 1445 & 15-minute & 3.36 \\
\hline & p75000_0195a.rdb & 01-12-95@2100 to 01-15-95@0015 & 15-minute & .96 \\
\hline & p75000_0195b.rdb & 01-22-95@ 1400 to 01-27-95@0730 & 15-minute & 2.32 \\
\hline & p75000_0395.rdb & 03-07-95@0400 to 03-16-95@0445 & 15-minute & 2.30 \\
\hline & p75000_0495.rdb & 03-27-95@0200 to 04-05-95 @ 1800 & 15-minute & 1.18 \\
\hline & p75000_0595.rdb & 05-27-95 @0800 to 06-01-95 @ 1145 & 15-minute & 2.20 \\
\hline \multirow[t]{12}{*}{08075400} & p75400_0191a.rdb & 01-09-91 @0100 to 01-10-91@ @ 1015 & 15-minute & 2.50 \\
\hline & p75400_0191b.rdb & 01-14-91@ @1500 to 01-15-91@0000 & 15-minute & 3.61 \\
\hline & p75400_0291.rdb & 02-04-91@0800 to 02-05-91@0500 & 15-minute & 1.63 \\
\hline & p75400_0491.rdb & 04-04-91@0100 to 04-06-91@0745 & 15-minute & 1.02 \\
\hline & p75400_0192.rdb & 01-17-92@0900 to 01-22-92@0000 & 15-minute & 1.16 \\
\hline & p75400_0292a.rdb & 02-02-92 @ 1800 to 02-05-92 @ 0145 & 15-minute & .50 \\
\hline & p75400_0292b.rdb & 02-22-92@0400 to 02-24-92 @ 2115 & 15-minute & 3.97 \\
\hline & p75400_0492.rdb & 04-17-92 @2000 to 04-22-92 @ 2015 & 15-minute & 4.27 \\
\hline & p75400_0592.rdb & 05-26-92 @2100 to 06-03-92 @ 1200 & 15-minute & 3.62 \\
\hline & p75400_0193.rdb & 01-07-93@0100 to 01-09-93 @ 2215 & 15-minute & 1.95 \\
\hline & p75400_0293.rdb & 02-25-93@ @ 1100 to 03-02-93@0515 & 15-minute & 3.88 \\
\hline & p75400_0493.rdb & 04-03-93@1600 to 04-08-93@0915 & 15-minute & 3.01 \\
\hline
\end{tabular}


Appendix 1. Description of precipitation data-Continued

\begin{tabular}{|c|c|c|c|c|}
\hline $\begin{array}{c}\text { USGS } \\
\text { station no. }\end{array}$ & File name & Period of unit-value data & Frequency & $\begin{array}{c}\text { Maximum } \\
\text { precipitation } \\
\text { intensity } \\
\text { (inches/hour) }\end{array}$ \\
\hline & p75400_0693.rdb & 06-17-93@1300 to 06-23-93@1430 & 15-minute & 1.82 \\
\hline & p75400_1093.rdb & 10-20-93@1200 to 10-20-93@2315 & 15-minute & 1.89 \\
\hline & p75400_0694.rdb & 06-18-94@1600 to 06-25-94 @1815 & 15-minute & 3.62 \\
\hline & p75400_1094.rdb & 10-15-94@1300 to 10-22-94 @ 1530 & 15-minute & .22 \\
\hline & p75400_0395.rdb & 03-13-95@0400 to 03-15-95@1945 & 15-minute & 1.76 \\
\hline & p75400_0495.rdb & 04-04-95@0700 to 04-06-95@0300 & 15-minute & 1.02 \\
\hline & p75400_0595.rdb & 05-27-95@0800 to 06-01-95@0815 & 15-minute & 1.14 \\
\hline \multirow[t]{18}{*}{08075500} & p75500_0191.rdb & 01-09-91@0200 to 01-15-91@1900 & 15-minute & 3.52 \\
\hline & p75500_0291.rdb & 02-04-91@0800 to 02-12-91@1515 & 15-minute & 1.37 \\
\hline & p75500_0491.rdb & 04-04-91@1000 to 04-06-91@0630 & 15-minute & 1.73 \\
\hline & p75500_0192.rdb & 01-17-92@0900 to 01-22-92 @0015 & 15-minute & 1.65 \\
\hline & p75500_0292a.rdb & 02-03-92@0300 to 02-05-92 @0145 & 15-minute & .58 \\
\hline & p75500_0292b.rdb & 02-22-92@0400 to 02-26-92 @0730 & 15-minute & 2.74 \\
\hline & p75500_0592.rdb & 05-26-92@2200 to 06-01-92 @1430 & 15-minute & 11.97 \\
\hline & p75500_0193.rdb & 01-04-93@1000 to 01-10-93@0045 & 15-minute & 1.43 \\
\hline & p75500_0293.rdb & 02-25-93@1100 to 03-02-93@0515 & 15-minute & 1.84 \\
\hline & p75500_0493.rdb & 04-03-93@1300 to 04-08-93@0100 & 15-minute & 1.46 \\
\hline & p75500_0693.rdb & 06-18-93@0600 to 06-26-93@0330 & 15-minute & .82 \\
\hline & p75500_1093.rdb & 10-20-93@1100 to 10-21-93@0230 & 15-minute & 4.49 \\
\hline & p75500_1193.rdb & 11-14-93@0500 to 11-16-93@1415 & 15-minute & 2.78 \\
\hline & p75500_0494.rdb & 04-30-94@0900 to 05-17-94@0215 & 15-minute & 2.68 \\
\hline & p75500_0694.rdb & 06-20-94@1600 to 06-24-94@1700 & 15-minute & .94 \\
\hline & p75500_1094.rdb & 10-15-94@1000 to 10-18-94@2200 & 15-minute & 3.90 \\
\hline & p75500_0395.rdb & 03-12-95@2300 to 03-13-95@1045 & 15-minute & 1.93 \\
\hline & p75500_0495.rdb & 04-04-95@0800 to 04-05-95 @1845 & 15-minute & .92 \\
\hline \multirow[t]{12}{*}{08075650} & p75650_0492.rdb & 04-05-92@0700 to 04-05-92@1730 & 15-minute & .83 \\
\hline & p75650_0592.rdb & 05-26-92 @2200 to 05-29-92 @0900 & 15-minute & 2.79 \\
\hline & p75650_0692.rdb & 06-01-92@1400 to 06-02-92 @1030 & 15-minute & 3.46 \\
\hline & p75650_0293.rdb & 02-25-93@1100 to 03-02-93@0515 & 15-minute & 1.62 \\
\hline & p75650_0393.rdb & 03-19-93@2300 to 03-22-93@2200 & 15-minute & 1.42 \\
\hline & p75650_0493.rdb & 04-03-93@1800 to 04-07-93@2300 & 15-minute & 2.99 \\
\hline & p75650_1093.rdb & 10-19-93@1200 to 10-21-93@0630 & 15-minute & 4.02 \\
\hline & p75650_1193.rdb & 11-08-93@1100 to 11-16-93@1500 & 15-minute & 2.81 \\
\hline & p75650_0194.rdb & 01-25-94@0100 to 01-27-94@1130 & 15-minute & .84 \\
\hline & p75650_1094.rdb & 10-15-94@0900 to 10-18-94@2000 & 15-minute & 3.48 \\
\hline & p75650_0495.rdb & 04-04-95@0700 to 04-05-95@1845 & 15-minute & 1.10 \\
\hline & p75650_0595.rdb & 05-29-95@0500 to 06-01-95@0645 & 15-minute & 1.63 \\
\hline \multirow[t]{4}{*}{08075730} & p75730_1089.rdb & 10-29-89@1200 to 11-01-89@2315 & 15-minute & 1.99 \\
\hline & p75730_0490.rdb & 04-26-90@1400 to 04-28-90@0815 & 15-minute & 2.23 \\
\hline & p75730_0890.rdb & 08-15-90@1600 to 08-15-90@1745 & 15-minute & 2.83 \\
\hline & p75730_0191.rdb & 01-14-91@2000 to 01-18-91@0230 & 15-minute & 1.60 \\
\hline
\end{tabular}


Appendix 1. Description of precipitation data-Continued

\begin{tabular}{|c|c|c|c|c|}
\hline $\begin{array}{c}\text { USGS } \\
\text { station no. }\end{array}$ & File name & Period of unit-value data & Frequency & $\begin{array}{l}\text { Maximum } \\
\text { precipitation } \\
\text { intensity } \\
\text { (inches/hour) }\end{array}$ \\
\hline & p75730_0291.rdb & 02-04-91 @0800 to 02-04-91 @ 1900 & 15-minute & 1.96 \\
\hline & p75730_0491.rdb & 04-04-91 @ 1000 to 04-05-91 @ 1515 & 15-minute & 2.11 \\
\hline & p75730_0591.rdb & 05-29-91 @ 1100 to 05-29-91 @ 1145 & 15-minute & 1.86 \\
\hline & p75730_1291.rdb & 12-18-91@2000 to 12-31-91@ 1430 & 15-minute & .09 \\
\hline & p75730_0492.rdb & 04-17-92@1900 to 04-19-92 @ 2230 & 15-minute & 2.78 \\
\hline & p75730_0592.rdb & 05-26-92@2200 to 05-28-92 @ 2115 & 15-minute & 3.04 \\
\hline & p75730_0692.rdb & 05-31-92@1400 to 06-06-92 @ 1415 & 15-minute & 1.92 \\
\hline & p75730_0293.rdb & 02-25-93@ @ 1100 to 03-01-93@ 1930 & 15-minute & 1.54 \\
\hline & p75730_0793.rdb & 07-15-93@1300 to 07-19-93@ 1530 & 15-minute & 2.05 \\
\hline & p75730_1093.rdb & 10-19-93@1300 to 10-20-93@2245 & 15-minute & 1.50 \\
\hline & p75730_1193.rdb & 11-14-93@0600 to 11-16-93@ 1415 & 15-minute & 1.52 \\
\hline & p75730_0194.rdb & 01-25-94@0200 to 01-29-94@ 1200 & 15-minute & 1.05 \\
\hline & p75730_1094.rdb & 10-15-94@0900 to 10-18-94@2000 & 15-minute & 4.88 \\
\hline & p75730_0495.rdb & 04-04-95 @0800 to 04-05-95 @ 1845 & 15-minute & 1.01 \\
\hline & p75730_0595.rdb & 05-27-95 @0900 to 06-01-95 @ 1430 & 15-minute & 3.07 \\
\hline \multirow[t]{19}{*}{08075770} & p75770_0390.rdb & 03-28-90 @0800 to 04-02-90 @ 1500 & 15-minute & 1.59 \\
\hline & p75770_0490.rdb & 04-26-90 @ 1300 to 04-27-90 @ 2145 & 15-minute & 2.47 \\
\hline & p75770_0790.rdb & 07-05-90@0900 to 07-07-90 @ 1145 & 15-minute & 2.68 \\
\hline & p75770_0191a.rdb & 01-09-91 @ 1200 to 01-10-91@ @ 1030 & 15-minute & 2.36 \\
\hline & p75770_0191b.rdb & 01-14-91@ @1600 to 01-19-91@0200 & 15-minute & 1.66 \\
\hline & p75770_0491a.rdb & 04-04-91@ @1000 to 04-05-91@ @ 1500 & 15-minute & 1.01 \\
\hline & p75770_0491b.rdb & 04-14-91@0800 to 04-17-91@0030 & 15-minute & 2.60 \\
\hline & p75770_1291.rdb & 12-21-91@0100 to 12-27-91@ 1515 & 15-minute & .95 \\
\hline & p75770_0592.rdb & 05-27-92@0600 to 05-28-92@ @2030 & 15-minute & 2.55 \\
\hline & p75770_0692.rdb & 05-31-92@ @ 1300 to 06-06-92 @ 1415 & 15-minute & 2.20 \\
\hline & p75770_0293.rdb & 02-25-93 @ 1000 to 03-02-93 @ 1115 & 15-minute & 2.07 \\
\hline & p75770_0393.rdb & 03-19-93 @2300 to 03-23-93@0215 & 15-minute & 3.22 \\
\hline & p75770_1093.rdb & 10-20-93@1200 to 10-21-93@0400 & 15-minute & 4.41 \\
\hline & p75770_1193.rdb & 11-14-93@0900 to 11-16-93@ 1345 & 15-minute & 2.07 \\
\hline & p75770_0594.rdb & 05-13-94 @ 1800 to 05-17-94@2100 & 15-minute & 1.67 \\
\hline & p75770_1094.rdb & 10-15-94@0900 to 10-19-94@0215 & 15-minute & 2.99 \\
\hline & p75770_0195.rdb & 01-18-95@0800 to 01-27-95@0900 & 15-minute & 2.72 \\
\hline & p75770_0495.rdb & 03-29-95@0800 to 04-05-95 @ 1730 & 15-minute & 1.63 \\
\hline & p75770_0595.rdb & 05-29-95@0700 to 06-01-95@ 1030 & 15-minute & 1.11 \\
\hline \multirow[t]{7}{*}{08075780} & p75780_1089.rdb & 10-29-89@0900 to 11-01-89@ 1000 & 15-minute & .20 \\
\hline & p75780_0290.rdb & 02-21-90@0800 to 02-21-90 @ 1445 & 15-minute & 2.26 \\
\hline & p75780_0390.rdb & 03-28-90@ @ 1000 to 03-30-90 @ 1200 & 15-minute & 1.34 \\
\hline & p75780_0490.rdb & 04-26-90 @ 1300 to 04-27-90 @ 2145 & 15-minute & 2.76 \\
\hline & p75780_0191a.rdb & 12-31-90@1800 to 01-10-91@ @ 1015 & 15-minute & 1.50 \\
\hline & p75780_0191b.rdb & 01-14-91@1400 to 01-15-91@0015 & 15 -minute & 1.35 \\
\hline & p75780_0191c.rdb & $01-18-91 @ 0600$ to $01-18-91 @ 1345$ & 15 -minute & 1.48 \\
\hline
\end{tabular}


Appendix 1. Description of precipitation data-Continued

\begin{tabular}{|c|c|c|c|c|}
\hline $\begin{array}{c}\text { USGS } \\
\text { station no. }\end{array}$ & File name & Period of unit-value data & Frequency & $\begin{array}{c}\text { Maximum } \\
\text { precipitation } \\
\text { intensity } \\
\text { (inches/hour) }\end{array}$ \\
\hline & p75780_0291.rdb & 02-04-91@0800 to 02-05-91@0530 & 15-minute & 0.52 \\
\hline & p75780_0491a.rdb & 04-04-91@0300 to 04-06-91@0730 & 15-minute & 1.87 \\
\hline & p75780_0491b.rdb & 04-14-91@0700 to 04-15-91@0645 & 15-minute & 1.82 \\
\hline & p75780_0691.rdb & 06-14-91@1600 to 06-18-91@2045 & 15-minute & 2.18 \\
\hline & p75780_1191.rdb & 11-17-91@0400 to 11-19-91@1500 & 15-minute & 2.43 \\
\hline & p75780_1291.rdb & $12-17-91 @ 0800$ to $12-23-91 @ 0100$ & 15-minute & .63 \\
\hline & p75780_0392.rdb & 03-04-92@0100 to 03-06-92@1015 & 15-minute & 1.08 \\
\hline & p75780_0492.rdb & 04-17-92@1300 to 04-19-92 @2115 & 15-minute & 2.64 \\
\hline & p75780_0592.rdb & 05-16-92@0400 to 05-20-92 @ 1645 & 15-minute & 2.08 \\
\hline & p75780_0692.rdb & 06-01-92@0400 to 06-02-92@0715 & 15-minute & 2.52 \\
\hline & p75780_0293.rdb & 02-25-93@0900 to 03-02-93@0230 & 15-minute & 2.22 \\
\hline & p75780_0393a.rdb & 02-28-93@2000 to 03-02-93@0230 & 15-minute & 1.22 \\
\hline & p75780_0393b.rdb & 03-19-93@2200 to 03-22-93@2130 & 15-minute & 1.52 \\
\hline & p75780_0493.rdb & 04-03-93@1200 to 04-07-93@2200 & 15-minute & 3.31 \\
\hline & p75780_0593.rdb & 05-23-93@1100 to 05-30-93@1915 & 15-minute & 1.82 \\
\hline & p75780_0693.rdb & 06-17-93@1800 to 06-26-93@1615 & 15-minute & 1.14 \\
\hline & p75780_1093.rdb & 10-19-93@1200 to 10-21-93@0330 & 15-minute & .92 \\
\hline & p75780_1193.rdb & 11-13-93@1100 to 11-16-93@1245 & 15-minute & 1.01 \\
\hline & p75780_0194.rdb & 01-25-94@1100 to 01-29-94@1630 & 15-minute & 1.55 \\
\hline & p75780_0294a.rdb & 02-09-94@1100 to 02-10-94@1800 & 15-minute & 1.52 \\
\hline & p75780_0294b.rdb & 02-20-94@1600 to 02-23-94@0700 & 15-minute & 1.10 \\
\hline & p75780_0594.rdb & 05-13-94@0800 to 05-17-94@1600 & 15-minute & 3.07 \\
\hline & p75780_0694.rdb & 06-18-94@1600 to 06-24-94@1800 & 15-minute & 1.34 \\
\hline & p75780_0894.rdb & 08-15-94@1700 to 08-22-94@1345 & 15-minute & 1.46 \\
\hline & p75780_1094.rdb & 10-15-94@0300 to 10-18-94@2345 & 15-minute & 2.52 \\
\hline & p75780_0195a.rdb & 01-12-95@2000 to 01-27-95@0700 & 15-minute & .99 \\
\hline & p75780_0195b.rdb & 01-22-95@1400 to 01-27-95@0700 & 15-minute & .99 \\
\hline & p75780_0395.rdb & 03-12-95@1900 to 03-17-95@0715 & 15-minute & 1.26 \\
\hline & p75780_0495.rdb & 04-04-95@0700 to 04-05-95@1745 & 15-minute & .71 \\
\hline & p75780_0595.rdb & 05-27-95@0800 to 06-02-95@1830 & 15-minute & 1.43 \\
\hline \multirow[t]{11}{*}{08076000} & p76000_0290.rdb & 02-21-90@1000 to 02-21-90@1500 & 15-minute & .79 \\
\hline & p76000_0390.rdb & 03-28-90@0900 to 03-30-90 @1230 & 15-minute & 2.34 \\
\hline & p76000_0490.rdb & 04-26-90@1300 to 04-28-90@0700 & 15-minute & 2.20 \\
\hline & p76000_0191a.rdb & $12-30-90 @ 0900$ to $01-10-91 @ 1600$ & 15-minute & 3.59 \\
\hline & p76000_0191b.rdb & 01-14-91@1400 to 01-19-91@0030 & 15-minute & 1.22 \\
\hline & p76000_0291.rdb & 02-04-91@0800 to 02-05-91@0330 & 15-minute & 1.26 \\
\hline & p76000_0491.rdb & 04-04-91@1000 to 04-19-91@1745 & 15-minute & 67 \\
\hline & p76000_0691.rdb & 06-13-91@1400 to 06-16-91@1900 & 15-minute & 2.08 \\
\hline & p76000_1191.rdb & 11-17-91@0600 to 11-19-91@1730 & 15-minute & 2.84 \\
\hline & p76000_1291.rdb & 12-17-91@0900 to 12-22-91@1930 & 15-minute & .96 \\
\hline & p76000_0392.rdb & 03-04-92@0100 to 03-09-92 @ 1645 & 15-minute & 2.96 \\
\hline
\end{tabular}


Appendix 1. Description of precipitation data-Continued

\begin{tabular}{|c|c|c|c|c|}
\hline $\begin{array}{c}\text { USGS } \\
\text { station no. }\end{array}$ & File name & Period of unit-value data & Frequency & $\begin{array}{l}\text { Maximum } \\
\text { precipitation } \\
\text { intensity } \\
\text { (inches/hour) }\end{array}$ \\
\hline & p76000_0293.rdb & 02-25-93@0900 to 03-02-93@0215 & 15-minute & 1.82 \\
\hline & p76000_0393.rdb & 03-22-93@ 1400 to 03-22-93 @ 2115 & 15-minute & 1.46 \\
\hline & p76000_0493.rdb & 04-03-93 @ 1200 to 04-07-93 @ 1830 & 15-minute & 3.07 \\
\hline & p76000_0693.rdb & 06-15-93@2300 to 06-18-93@ 1330 & 15-minute & .19 \\
\hline & p76000_1093.rdb & 10-19-93@1600 to 10-21-93@0400 & 15-minute & 1.06 \\
\hline & p76000_1193.rdb & 11-13-93@1500 to 11-16-93@ 1315 & 15-minute & 3.33 \\
\hline & p76000_0594.rdb & 05-13-94@1800 to 05-17-94@ @ 1915 & 15-minute & 4.41 \\
\hline & p76000_0894.rdb & 08-17-94@ @ 1700 to 08-17-94@ 1815 & 15-minute & 1.29 \\
\hline & p76000_1094.rdb & 10-15-94@0400 to 10-19-94@1900 & 15-minute & 1.09 \\
\hline & p76000_0195.rdb & 01-22-95@1400 to 01-27-95@0130 & 15-minute & .75 \\
\hline & p76000_0395.rdb & 03-13-95@0200 to 03-16-95 @ 1715 & 15-minute & 1.94 \\
\hline & p76000_0495.rdb & 04-04-95@0400 to 04-10-95@2215 & 15-minute & 1.30 \\
\hline \multirow[t]{23}{*}{08076180} & p76180_0390.rdb & 03-28-90@0900 to 04-01-90 @ 1645 & 15-minute & 2.26 \\
\hline & p76180_0490.rdb & 04-26-90@1300 to 04-27-90 @ 2115 & 15-minute & 3.31 \\
\hline & p76180_0590.rdb & 05-03-90@1500 to 05-03-90@ @ 1845 & 15-minute & 1.97 \\
\hline & p76180_0191.rdb & 01-06-91@0800 to 01-18-91@ @ 1500 & 15-minute & 2.83 \\
\hline & p76180_0291.rdb & 02-04-91 @0800 to 02-04-91 @ 1900 & 15-minute & 1.05 \\
\hline & p76180_0491.rdb & 04-04-91@1200 to 04-19-91@ @ 1730 & 15-minute & 1.42 \\
\hline & p76180_0691.rdb & 06-13-91@ @1300 to 06-21-91@ 1915 & 15-minute & 2.05 \\
\hline & p76180_1191.rdb & 11-17-91@0400 to 11-19-91@ @ 1730 & 15-minute & 2.53 \\
\hline & p76180_1291.rdb & 12-17-91@0700 to 12-27-91@ @1215 & 15-minute & 1.03 \\
\hline & p76180_0392.rdb & 03-04-92@0100 to 03-04-92 @ 1630 & 15-minute & 4.72 \\
\hline & p76180_0592.rdb & 05-27-92 @0800 to 06-06-92 @ 1330 & 15-minute & 2.21 \\
\hline & p76180_0293.rdb & 02-25-93@0900 to 03-01-93@ 1900 & 15-minute & 1.44 \\
\hline & p76180_0393.rdb & 03-22-93 @ 1600 to 03-22-93 @ 2245 & 15-minute & 1.38 \\
\hline & p76180_0493.rdb & 04-03-93 @ 1200 to 04-07-93 @ 1830 & 15-minute & 3.01 \\
\hline & p76180_0693.rdb & 06-15-93 @2300 to 06-28-93 @ 1515 & 15-minute & 2.78 \\
\hline & p76180_1093.rdb & 10-20-93@1200 to 10-21-93@0530 & 15-minute & 2.60 \\
\hline & p76180_1193.rdb & 11-13-93 @ 1100 to 11-16-93@ 1330 & 15-minute & 4.25 \\
\hline & p76180_0594a.rdb & 05-13-94@0800 to 05-16-94@0430 & 15-minute & 4.25 \\
\hline & p76180_0594b.rdb & 05-28-94@ @ 1400 to 06-03-94@ 1300 & 15-minute & 1.60 \\
\hline & p76180_1095.rdb & 10-15-94@1000 to 10-10-94@0130 & 15-minute & 4.57 \\
\hline & p76180_0195.rdb & 01-22-95@ 1400 to 01-27-95@0130 & 15-minute & .84 \\
\hline & p76180_0395.rdb & 03-13-95@0700 to 03-16-95@ 1630 & 15-minute & 1.74 \\
\hline & p76180_0495.rdb & 04-04-95@0800 to 04-11-95@0930 & 15-minute & 1.59 \\
\hline \multirow[t]{6}{*}{08076500} & p76500_1089.rdb & 10-29-89@0800 to 11-01-89 @ 1700 & 15-minute & 1.76 \\
\hline & p76500_0390.rdb & 03-28-90 @0900 to 04-01-90 @ 1645 & 15-minute & 2.36 \\
\hline & p76500_0490.rdb & 04-26-90@1300 to 04-28-90@0500 & 15-minute & 2.52 \\
\hline & p76500_0191.rdb & 01-14-91@0900 to 01-19-91@0045 & 15-minute & 2.00 \\
\hline & p76500_0291.rdb & 02-04-91 @0800 to 02-04-91 @ 1845 & 15 -minute & .68 \\
\hline & p76500_0491.rdb & 04-04-91@0900 to 04-18-91@2330 & 15 -minute & 2.33 \\
\hline
\end{tabular}


Appendix 1. Description of precipitation data-Continued

\begin{tabular}{|c|c|c|c|c|}
\hline $\begin{array}{c}\text { USGS } \\
\text { station no. }\end{array}$ & File name & Period of unit-value data & Frequency & $\begin{array}{l}\text { Maximum } \\
\text { precipitation } \\
\text { intensity } \\
\text { (inches/hour) }\end{array}$ \\
\hline & p76500_0591.rdb & 05-15-91@ @1400 to 05-18-91 @2145 & 15-minute & 2.06 \\
\hline & p76500_0691.rdb & 06-11-91 @ 1900 to 06-18-91@2015 & 15-minute & 2.68 \\
\hline & p76500_1191.rdb & 11-17-91@0400 to 11-19-91@ 1745 & 15-minute & 3.20 \\
\hline & p76500_1291.rdb & 12-21-91@0500 to 12-22-91@ 1830 & 15-minute & 60 \\
\hline & p76500_0392.rdb & 03-04-92@0100 to 03-06-92@0115 & 15-minute & 3.01 \\
\hline & p76500_0492.rdb & 04-17-92 @ 1200 to 04-19-92 @ 2300 & 15-minute & 2.30 \\
\hline & p76500_0293.rdb & 02-25-93@0900 to 03-02-93 @0200 & 15-minute & 2.06 \\
\hline & p76500_0393.rdb & 03-19-93 @ 2200 to 03-22-93 @2115 & 15-minute & 1.32 \\
\hline & p76500_0493.rdb & 04-03-93 @1200 to 04-07-93@ @ 1815 & 15-minute & 2.38 \\
\hline & p76500_0693.rdb & 06-17-93 @ 1100 to 06-26-93 @ 1400 & 15-minute & 1.19 \\
\hline & p76500_1093.rdb & 10-20-93@1200 to 10-21-93@0330 & 15-minute & 1.70 \\
\hline & p76500_1193.rdb & 11-13-93@1400 to 11-16-93@2045 & 15-minute & 4.47 \\
\hline & p76500_0594.rdb & 05-13-94 @ 1800 to 05-17-94 @ 1545 & 15-minute & 4.72 \\
\hline & p76500_0894.rdb & 08-15-94 @ 1800 to 08-17-94 @ 1830 & 15-minute & 1.89 \\
\hline & p76500_1094.rdb & 10-15-94@0900 to 10-18-94@ 1945 & 15-minute & 1.92 \\
\hline & p76500_0195.rdb & 01-22-95@1400 to 01-26-95@2200 & 15-minute & 2.31 \\
\hline & p76500_0395.rdb & 03-12-95 @ 2300 to 03-16-95 @ 1615 & 15-minute & 1.73 \\
\hline & p76500_0495.rdb & 04-04-95 @0800 to 04-11-95@0415 & 15-minute & 1.18 \\
\hline \multirow[t]{13}{*}{08076700} & p76700_0191.rdb & 01-09-91@1300 to 01-18-91 @ 1430 & 15-minute & 2.41 \\
\hline & p76700_0291.rdb & 02-04-91 @0900 to 02-05-91 @ 1215 & 15-minute & 1.38 \\
\hline & p76700_0691.rdb & 06-11-91 @ 1900 to 06-18-91 @2015 & 15-minute & 2.37 \\
\hline & p76700_0293.rdb & 02-25-93 @ 1000 to 03-02-93 @0215 & 15-minute & 2.16 \\
\hline & p76700_0493.rdb & 04-03-93 @ 1200 to 04-07-93 @ 2145 & 15-minute & 2.21 \\
\hline & p76700_0693.rdb & 06-17-93@0700 to 06-26-93 @ 1330 & 15-minute & 2.04 \\
\hline & p76700_1093.rdb & 10-20-93@1200 to 10-21-93@0330 & 15-minute & 6.14 \\
\hline & p76700_1193.rdb & 11-13-93 @ 1500 to 11-16-93 @ 1400 & 15-minute & 2.09 \\
\hline & p76700_0594.rdb & 05-15-94@0700 to 05-16-94@0200 & 15-minute & 4.72 \\
\hline & p76700_1094.rdb & $10-15-94 @ 1000$ to $10-19-94 @ 1145$ & 15-minute & 2.83 \\
\hline & p76700_0195.rdb & 01-18-95 @0800 to 01-26-95@ 1630 & 15-minute & 2.18 \\
\hline & p76700_0395.rdb & 03-13-95 @0700 to 03-16-95@ 1730 & 15-minute & 1.73 \\
\hline & p76700_0495.rdb & 04-04-95 @0900 to 04-04-95 @ 1515 & 15-minute & .53 \\
\hline
\end{tabular}




\section{Appendix 2-Description of Surface-Water Data}




\section{Appendix 2. Description of surface-water data}

\begin{tabular}{|c|c|c|c|c|c|c|}
\hline $\begin{array}{l}\text { USGS } \\
\text { station no. }\end{array}$ & File name & $\begin{array}{l}\text { Period of unit-value data } \\
\text { (gage height and } \\
\text { (or) discharge }\end{array}$ & $\begin{array}{c}\text { Fre- } \\
\text { quency }\end{array}$ & $\begin{array}{c}\text { Maximum } \\
\text { gage height } \\
\text { (feet above } \\
\text { datum) }\end{array}$ & $\begin{array}{c}\text { Maximum } \\
\text { discharge } \\
\text { (cubic feet/ } \\
\text { second) }\end{array}$ & Comments \\
\hline \multirow[t]{19}{*}{08073700} & quv73700_0191.rdb & 01-09-91@1200 to 01-20-91 @ 1300 & 15-minute & & 2,540 & \\
\hline & quv73700_0291.rdb & 02-04-91@0800 to 02-06-91 @ 1100 & 15-minute & & 2,440 & \\
\hline & quv73700_0491a.rdb & 04-04-91@0800 to 04-08-91 @ 1200 & 15-minute & & 2,580 & \\
\hline & quv73700_0491b.rdb & 04-14-91@0800 to 04-17-91@ 1000 & 15-minute & & 2,560 & \\
\hline & quv73700_1291.rdb & 12-21-91@0600 to 12-26-91@0600 & 15-minute & & 3,710 & \\
\hline & quv73700_0392.rdb & 03-03-92 @ 1200 to 03-09-92 @ 1800 & 15-minute & & 7,500 & \\
\hline & quv73700_0492.rdb & 04-13-92@ @ 1000 to 04-20-92 @ 1300 & 15-minute & & 4,120 & \\
\hline & quv73700_0892.rdb & 08-02-92@ 1800 to 08-04-92 @0900 & 15-minute & & 3,560 & \\
\hline & quv73700_0293.rdb & 02-25-93@0800 to 03-03-93@ 1200 & 15-minute & & 3,860 & \\
\hline & quv73700_0393.rdb & 03-19-93 @ 1000 to 03-29-93 @ 1100 & 15 -minute & & 3,350 & \\
\hline & quv73700_0693.rdb & 06-19-93@0500 to 06-23-93 @ 1000 & 15-minute & & 4,550 & \\
\hline & quv73700_1093.rdb & 10-20-93@0900 to 10-27-93@2000 & 15-minute & & 2,080 & \\
\hline & quv73700_1193.rdb & 11-13-93@1000 to 11-24-93@ 1000 & 15-minute & & 2,330 & \\
\hline & quv73700_0694.rdb & 06-17-94@1000 to 06-30-94 @0800 & 15-minute & & 2,320 & \\
\hline & quv73700_0894.rdb & 08-21-94@ 1000 to 08-27-94@ 1000 & 15-minute & & 2,570 & \\
\hline & quv73700_1094.rdb & 10-15-94@0700 to 10-22-94 @ 1300 & 15-minute & & 4,940 & \\
\hline & quv73700_0195.rdb & 01-26-95@0200 to 02-05-95 @ 2300 & 15-minute & & 3,110 & \\
\hline & quv73700_0395.rdb & 03-13-95@0500 to 03-16-95 @ 1100 & 15-minute & & 2,310 & \\
\hline & quv73700_0495.rdb & 04-03-95 @ 1200 to 04-07-95 @ 1000 & 15-minute & & 3,550 & \\
\hline \multirow[t]{20}{*}{08074000} & quv74000_0191.rdb & 01-09-91@ @ 1200 to 01-21-91 @ 1200 & 60-minute & & 4,630 & \\
\hline & quv74000_0291.rdb & 02-04-91@0800 to 02-06-91 @ 1700 & 60-minute & & 4,170 & \\
\hline & quv74000_0491a.rdb & 04-04-91@ @ 1200 to 04-08-91 @ 1200 & 60 -minute & & 4,840 & \\
\hline & quv74000_0491b.rdb & 04-14-91@0800 to 04-17-91 @ 1200 & 60-minute & & 4,480 & \\
\hline & quv74000_1291.rdb & 12-21-91@0500 to 12-26-91@0700 & 60-minute & & 5,220 & \\
\hline & quv74000_0392.rdb & 03-03-92@ @2100 to 03-09-92 @ 1800 & 60-minute & & 12,500 & No data 03-04@1300-1600 \\
\hline & quv74000_0492.rdb & 04-13-92 @ 1700 to 04-19-92 @ 2000 & 60-minute & & 6,120 & \\
\hline & quv74000_0592.rdb & 05-16-92@0500 to 05-19-92 @ 1700 & 60-minute & & 5,390 & \\
\hline & quv74000_0293.rdb & 02-25-93@0800 to 03-03-93 @ 1700 & 15 -minute & & 6,710 & No data 02-25@0900-1330 \\
\hline & quv74000_0393.rdb & 03-19-93@2200 to 03-31-93@ 1700 & 15-minute & & 6,570 & $\begin{array}{l}\text { No data 03-23 @ 0715-2100; 03-30 } \\
@ 1515 \text { to 03-31 @ 1600 }\end{array}$ \\
\hline & quv74000_0493.rdb & 04-07-93@ @ 1100 to 04-15-93 @ 1700 & 15-minute & & 4,220 & \\
\hline & quv74000_0693.rdb & 06-19-93@1000 to 06-23-93@ @2300 & 15-minute & & 6,610 & $\begin{array}{l}\text { No data 06-22@1215 to 06-23 } \\
@ 1330\end{array}$ \\
\hline & quv74000_1093.rdb & 10-20-93@1100 to 10-26-93@ 1700 & 15-minute & & 4,400 & \\
\hline & quv74000_1193.rdb & 11-13-93@1600 to 11-24-93@ 1500 & 15-minute & & 5,890 & \\
\hline & quv74000_0694.rdb & 06-20-94@1930 to 06-30-94 @ 1100 & 15-minute & & 2,720 & \\
\hline & quv74000_0894.rdb & 08-21-94@ @1100 to 08-27-94 @ 1100 & 15-minute & & 3,580 & $\begin{array}{l}\text { No data 08-23@1715 to 08-25 } \\
@ 1445\end{array}$ \\
\hline & quv74000_1094.rdb & 10-15-94@1915 to 10-22-94@2145 & 15 -minute & & 8,450 & \\
\hline & quv74000_0195.rdb & 01-26-95@0900 to 02-06-95 @ 1200 & 15-minute & & 5,540 & \\
\hline & quv74000_0395.rdb & 03-13-95 @0400 to 03-16-95 @ 2000 & 15-minute & & 4,240 & \\
\hline & quv74000_0495.rdb & 04-04-95 @ 0000 to 04-07-95 @ 1600 & 15-minute & & 5,050 & \\
\hline
\end{tabular}


Appendix 2. Description of surface-water data-Continued

\begin{tabular}{|c|c|c|c|c|c|c|}
\hline $\begin{array}{c}\text { USGS } \\
\text { station no. }\end{array}$ & File name & $\begin{array}{l}\text { Period of unit-value data } \\
\text { (gage height and } \\
\text { (or) discharge }\end{array}$ & $\begin{array}{c}\text { Fre- } \\
\text { quency }\end{array}$ & $\begin{array}{c}\text { Maximum } \\
\text { gage height } \\
\text { (feet above } \\
\text { datum) }\end{array}$ & $\begin{array}{c}\text { Maximum } \\
\text { discharge } \\
\text { (cubic feet/ } \\
\text { second) }\end{array}$ & Comments \\
\hline \multirow[t]{25}{*}{08074020} & quv74020_1089.rdb & 10-29-89@1200 to 10-30-89@0600 & 30-minute & & 1,940 & \\
\hline & quv74020_0290.rdb & 02-21-90@0900 to 02-22-90@1000 & 30-minute & & 3,420 & \\
\hline & quv74020_0490.rdb & 04-26-90@1200 to 04-28-90@2000 & 30-minute & & 1,260 & \\
\hline & quv74020_0191a.rdb & 01-09-91@1400 to 01-11-91@0400 & 30-minute & & 1,350 & \\
\hline & quv74020_0191b.rdb & 01-18-91@0700 to 01-19-91@0800 & 30-minute & & 2,320 & \\
\hline & quv74020_0291.rdb & 02-04-91@1100 to 02-05-91@0900 & 30-minute & & 2,120 & \\
\hline & quv74020_0491a.rdb & 04-04-91@ @ 1200 to 04-07-91@0100 & 30-minute & & 2,480 & \\
\hline & quv74020_0491b.rdb & 04-14-91@0700 to 04-16-91@0300 & 30 -minute & & 4,440 & \\
\hline & quv74020_1191.rdb & 11-17-91@0700 to 11-18-91@1800 & 30-minute & & 3,180 & \\
\hline & quv74020_1291.rdb & $12-21-91 @ 0700$ to $12-27-91 @ 2200$ & 30 -minute & & 3,930 & \\
\hline & quv74020_0492.rdb & 04-17-92@1500 to 04-18-92 @2200 & 30-minute & & 3,020 & \\
\hline & quv74020_0592.rdb & 05-16-92@1100 to 05-18-92@0400 & 30-minute & & 2,640 & \\
\hline & quv74020_0692.rdb & 06-01-92@1500 to 06-03-92@1700 & 30-minute & & 2,930 & \\
\hline & quv74020_0393a.rdb & 03-01-93@0900 to 03-03-93@0300 & 30-minute & & 4,520 & \\
\hline & quv74020_0393b.rdb & 03-22-93@1600 to 03-23-93@2300 & 30-minute & & 2,280 & \\
\hline & quv74020_0493.rdb & 04-03-93@1100 to 04-05-93@0900 & 30-minute & & 2,200 & \\
\hline & quv74020_0693.rdb & 06-19-93@1400 to 06-23-93@0300 & 30-minute & & 2,990 & \\
\hline & quv74020_1193.rdb & 11-16-93@0800 to 11-17-93@0600 & 30-minute & & 1,470 & \\
\hline & quv74020_0294.rdb & 02-10-94@0800 to 02-11-94@0600 & 30-minute & & 1,800 & \\
\hline & quv74020_0594.rdb & 05-16-94@0000 to 05-16-94@1130 & 30-minute & & 1,890 & No data after 05-16@1130 \\
\hline & quv74020_0694.rdb & 06-23-94@1200 to 06-25-94@0300 & 30-minute & & 1,380 & \\
\hline & quv74020_0894.rdb & 08-05-94@1200 to 08-06-94@0900 & 30-minute & & 1,380 & \\
\hline & quv74020_1094.rdb & 10-17-94@0800 to 10-19-94@1500 & 30-minute & & 3,980 & \\
\hline & quv74020_0195.rdb & 01-26-95@1500 to 01-27-95@1800 & 30-minute & & 1,430 & \\
\hline & quv74020_0495.rdb & 04-04-95@0700 to 04-05-95@0600 & 30-minute & & 1,320 & \\
\hline \multirow[t]{16}{*}{08074150} & quv74150_1089.rdb & 10-29-89@1200 to 10-31-89@1500 & 30-minute & & 1,120 & \\
\hline & quv74150_0290.rdb & 02-21-90@0900 to 02-24-90@0300 & 30-minute & & 515 & \\
\hline & quv74150_0490.rdb & 04-26-90@0600 to 04-29-90@1900 & 30-minute & & 325 & \\
\hline & quv74150_0890.rdb & 08-14-90@1900 to 08-17-90@0100 & 30-minute & & 537 & \\
\hline & quv74150_0291.rdb & 02-04-91@0900 to 02-08-91@0300 & 30-minute & & 505 & \\
\hline & quv74150_0491a.rdb & 04-04-91@0800 to 04-08-91@0100 & 30-minute & & 635 & \\
\hline & quv74150_0491b.rdb & 04-14-91@0700 to 04-17-91@1500 & 30-minute & & 813 & \\
\hline & quv74150_0691.rdb & 06-14-91@1600 to 06-19-91 @2200 & 30-minute & & 1,180 & \\
\hline & quv74150_0392.rdb & 03-04-92@0000 to 03-08-92@0500 & 30-minute & & 2,780 & \\
\hline & quv74150_0492.rdb & 04-17-92@0700 to 04-19-92 @2000 & 30-minute & & 784 & \\
\hline & quv74150_0692.rdb & 06-01-92@1400 to 06-04-92 @2300 & 30-minute & & 851 & \\
\hline & quv74150_0393.rdb & 03-01-93@0700 to 03-04-93@0700 & 30-minute & & 954 & \\
\hline & quv74150_0493.rdb & 04-03-93@1000 to 04-10-93@0800 & 30-minute & & 620 & \\
\hline & quv74150_0693.rdb & 06-17-93@1500 to 06-23-93@2100 & 30-minute & & 732 & \\
\hline & quv74150_1193.rdb & 11-16-93@0200 to 11-18-93@2000 & 30-minute & & 387 & \\
\hline & quv74150_0494.rdb & 04-30-94@0200 to 05-04-94@0800 & 30-minute & & 537 & \\
\hline
\end{tabular}


Appendix 2. Description of surface-water data-Continued

\begin{tabular}{|c|c|c|c|c|c|c|}
\hline $\begin{array}{c}\text { USGS } \\
\text { station no. }\end{array}$ & File name & $\begin{array}{l}\text { Period of unit-value data } \\
\text { (gage height and } \\
\text { (or) discharge }\end{array}$ & $\begin{array}{c}\text { Fre- } \\
\text { quency }\end{array}$ & $\begin{array}{l}\text { Maximum } \\
\text { gage height } \\
\text { (feet above } \\
\text { datum) }\end{array}$ & $\begin{array}{c}\text { Maximum } \\
\text { discharge } \\
\text { (cubic feet } / \\
\text { second) }\end{array}$ & Comments \\
\hline & quv74150_0694.rdb & 06-20-94@1500 to 06-27-94@0800 & 30-minute & & 499 & \\
\hline & quv74150_0894a.rdb & 08-16-94@ @ 1400 to 08-20-94 @ 2200 & 30-minute & & 581 & \\
\hline & quv74150_0894b.rdb & 08-21-94@1100 to 08-26-94@ @ 1000 & 30-minute & & 598 & \\
\hline & quv74150_1094.rdb & 10-15-94@0300 to 10-22-94@ 2100 & 30-minute & & 773 & \\
\hline & quv74150_0195.rdb & 01-25-95@2200 to 01-30-95 @ 1100 & 30-minute & & 624 & \\
\hline & quv74150_0395.rdb & 03-13-95@0500 to 03-18-95 @ 1200 & 30-minute & & 462 & \\
\hline & quv74150_0495.rdb & 04-04-95@0400 to 04-09-95 @ 2300 & 30-minute & & 570 & \\
\hline \multirow[t]{26}{*}{08074250} & quv74250_1089.rdb & $10-29-89 @ 1200$ to $11-01-89 @ 0600$ & 15 -minute & & 3,130 & \\
\hline & quv74250_0290.rdb & 02-21-90@0700 to 02-23-90 @ 2300 & 15-minute & & 1,880 & \\
\hline & quv74250_0490.rdb & 04-26-90 @ 1300 to 04-29-90 @ 1500 & 15-minute & & 2,960 & \\
\hline & quv74250_0890.rdb & 08-14-90 @ 1900 to 08-16-90 @ 2000 & 15-minute & & 2,210 & \\
\hline & quv74250_0191.rdb & 01-14-91@ @ 1300 to 01-21-91 @ 0200 & 15 -minute & & 2,360 & \\
\hline & quv74250_0291.rdb & 02-04-91@0800 to 02-06-91@ 2200 & 15-minute & & 2,650 & \\
\hline & quv74250_0491.rdb & 04-10-91@0700 to 04-17-91@0600 & 15-minute & & 2,330 & \\
\hline & quv74250_0691.rdb & 06-16-91@ @ 1700 to 06-18-91 @ 2000 & 15 -minute & & 4,540 & \\
\hline & quv74250_1191.rdb & 11-17-91@0100 to 11-20-91@2300 & 15-minute & & 2,810 & \\
\hline & quv74250_0392.rdb & 03-04-92@0100 to 03-07-92 @ 1100 & 15-minute & & 7,580 & \\
\hline & quv74250_0492.rdb & 04-17-92 @ 1200 to 04-19-92 @ 1100 & 15-minute & & 3,370 & \\
\hline & quv74250_0592.rdb & 05-16-92@0300 to 05-19-92 @ 2300 & 15-minute & & 3,110 & \\
\hline & quv74250_0293.rdb & 02-28-93@2100 to 03-04-93@0900 & 15-minute & & 3,320 & \\
\hline & quv74250_0393.rdb & 03-22-93@1400 to 03-25-93@ 1200 & 15-minute & & 2,450 & \\
\hline & quv74250_0493.rdb & 04-07-93@1300 to 04-09-93@ 1100 & 15-minute & & 3,320 & \\
\hline & quv74250_0693.rdb & 06-17-93@1500 to 06-24-93@ 1000 & 15-minute & & 1,930 & \\
\hline & quv74250_1193.rdb & 11-16-93@0300 to 11-18-93@ 1000 & 15-minute & & 1,250 & \\
\hline & quv74250_0494.rdb & 04-30-94@0200 to 05-04-94@0600 & 15-minute & & 1,890 & \\
\hline & quv74250_0694.rdb & 06-23-94@1300 to 06-26-94@ @ 1000 & 15-minute & & 1,720 & \\
\hline & quv74250_0894a.rdb & 08-17-94@1700 to 08-20-94@0600 & 15-minute & & 3,800 & \\
\hline & quv74250_0894b.rdb & 08-22-94@0400 to 08-25-94@ 2100 & 15-minute & & 3,530 & \\
\hline & quv74250_1094.rdb & 10-15-94@0800 to 10-23-94@0900 & 15 -minute & & 2,690 & \\
\hline & quv74250_0195a.rdb & 01-12-95@1100 to 01-16-95 @0800 & 15-minute & & 2,700 & \\
\hline & quv74250_0195b.rdb & 01-25-95@2200 to 01-30-95 @ 1000 & 15-minute & & 2,200 & \\
\hline & quv74250_0395.rdb & 03-13-95@0400 to 03-15-95@ @ 1000 & 15-minute & & 2,490 & \\
\hline & quv74250_0495.rdb & 04-04-95@0400 to 04-08-95 @ 1000 & 15-minute & & 2,340 & \\
\hline \multirow[t]{8}{*}{08074500} & quv74500_1089.rdb & 10-29-89@1200 to 11-02-89@ 1000 & 30-minute & & 8,550 & \\
\hline & quv74500_0290.rdb & 02-21-90@0700 to 02-24-90 @ 1200 & 30-minute & & 4,230 & \\
\hline & quv74500_0490.rdb & 04-26-90@1100 to 05-01-90@ @2330 & 30-minute & & 6,920 & \\
\hline & quv74500_0191.rdb & 01-14-91@0800 to 01-21-91@ 1000 & 30-minute & & 6,500 & \\
\hline & quv74500_0291.rdb & 02-04-91@0700 to 02-07-91 @ 1000 & 30-minute & & 5,700 & \\
\hline & quv74500_0491.rdb & 04-10-91@ @1300 to 04-17-91@0600 & 30-minute & & 8,340 & $\begin{array}{l}\text { Many values missing 04-16@1230 } \\
\text { to 04-17@0600 }\end{array}$ \\
\hline & quv74500_0691.rdb & 06-14-91@1700 to 06-19-91@ 2300 & 30-minute & & 9,310 & \\
\hline & quv74500_1191.rdb & 11-17-91@0300 to 11-21-91@ 1000 & 30-minute & & 9,630 & \\
\hline
\end{tabular}


Appendix 2. Description of surface-water data-Continued

\begin{tabular}{|c|c|c|c|c|c|c|}
\hline $\begin{array}{c}\text { USGS } \\
\text { station no. }\end{array}$ & File name & $\begin{array}{l}\text { Period of unit-value data } \\
\text { (gage height and } \\
\text { (or) discharge }\end{array}$ & $\begin{array}{c}\text { Fre- } \\
\text { quency }\end{array}$ & $\begin{array}{c}\text { Maximum } \\
\text { gage height } \\
\text { (feet above } \\
\text { datum) }\end{array}$ & $\begin{array}{c}\text { Maximum } \\
\text { discharge } \\
\text { (cubic feet } / \\
\text { second) }\end{array}$ & Comments \\
\hline & quv74500_1291.rdb & 12-21-91@0100 to 12-30-91@2300 & 30 -minute & & 10,400 & \\
\hline & quv74500_0392.rdb & 03-03-92 @ 2000 to 03-09-92 @ 2000 & 30 -minute & & 25,100 & \\
\hline & quv74500_0592.rdb & 05-16-92@0500 to 05-20-92 @ 1100 & 30-minute & & 9,190 & \\
\hline & quv74500_0293.rdb & 02-28-93@2000 to 03-05-93 @ 2200 & 30 -minute & & 11,200 & \\
\hline & quv74500_0393.rdb & 03-22-93@1400 to 03-26-93 @ 2100 & 30 -minute & & 9,180 & \\
\hline & quv74500_0493.rdb & 04-07-93@1200 to 04-11-93 @ 1200 & 30-minute & & 7,300 & \\
\hline & quv74500_0693.rdb & 06-17-93@1000 to 06-24-93 @ 2100 & 30-minute & & 4,330 & \\
\hline & quv74500_1193.rdb & 11-14-93@0200 to 11-18-93@ 1700 & 30-minute & & 6,850 & \\
\hline & quv74500_0294.rdb & 02-20-94@1800 to 02-25-94 @ 2000 & 30-minute & & 3,860 & \\
\hline & quv74500_0694.rdb & 06-20-94@1400 to 06-27-94 @ 1100 & 30-minute & & 5,270 & \\
\hline & quv74500_0894a.rdb & 08-15-94@ @ 1700 to 08-20-94@ 1100 & 30-minute & & 5,240 & \\
\hline & quv74500_0894b.rdb & 08-21-94@0800 to 08-26-94@0900 & 30 -minute & & 5,410 & \\
\hline & quv74500_1094.rdb & 10-15-94@0800 to 10-25-94@0900 & 30-minute & & 11,800 & \\
\hline & quv74500_0195.rdb & 01-25-95@ @ 1900 to 01-31-95@ 1900 & 30-minute & & 7,420 & \\
\hline & quv74500_0395.rdb & 03-12-95@1100 to 03-18-95 @ 1100 & 30 -minute & & 6,250 & \\
\hline & quv74500_0495.rdb & 04-04-95@0700 to 04-10-95@0900 & 30-minute & & 5,240 & \\
\hline \multirow[t]{11}{*}{08074540} & ghuv74540_0191.rdb & 01-10-91@0800 to 01-10-91@ 1200 & 15 -minute & 33.84 & & \\
\hline & ghuv74540_0491a.rdb & 04-05-91@ @ 1200 to 04-05-91 @ 1900 & 15 -minute & 31.91 & & \\
\hline & ghuv74540_0491b.rdb & 04-14-91@1300 to 04-14-91 @ 1800 & 15-minute & 32.19 & & \\
\hline & ghuv74540_0691.rdb & 06-16-91@ @ 1700 to 06-16-91 @ 2300 & 15 -minute & 32.80 & & \\
\hline & ghuv74540_1191.rdb & 11-17-91@0600 to 11-17-91@ 1400 & 15-minute & 36.24 & & \\
\hline & ghuv74540_0392.rdb & 03-04-92 @ 1100 to 03-04-92 @ 1900 & 15 -minute & 43.17 & & No data after 03-04@1900 \\
\hline & ghuv74540_0393.rdb & 03-22-93@2000 to 03-23-93 @ 0000 & 15-minute & 38.25 & & \\
\hline & ghuv74540_0693.rdb & 06-26-93@0100 to 06-26-93 @ 0600 & 15-minute & 29.40 & & \\
\hline & ghuv74540_1093.rdb & 10-20-93@1600 to 10-20-93 @ 2300 & 15 -minute & 29.29 & & \\
\hline & ghuv74540_0894.rdb & 08-17-94@1600 to 08-17-94 @ 2000 & 15-minute & 29.53 & & \\
\hline & ghuv74540_0495.rdb & 04-04-95@1000 to 04-04-95 @ 1800 & 15 -minute & 29.46 & & \\
\hline \multirow[t]{14}{*}{08074760} & ghuv74760_0191.rdb & 01-14-91@2100 to 01-15-91 @ 1400 & 30-minute & 13.27 & & \\
\hline & ghuv74760_0291.rdb & 02-04-91@ @ 1000 to 02-05-91@0500 & 30-minute & 13.27 & & \\
\hline & ghuv74760_0491a.rdb & 04-05-91@0800 to 04-06-91@0800 & 30 -minute & 13.08 & & \\
\hline & ghuv74760_0491b.rdb & 04-14-91@1000 to 04-15-91 @ 1100 & 30-minute & 13.40 & & \\
\hline & ghuv74760_0292.rdb & 02-22-92@0500 to 02-23-92 @ 0100 & 30-minute & 14.10 & & \\
\hline & ghuv74760_0392.rdb & 03-04-92@1100 to 03-05-92 @ 1900 & 30 -minute & 21.16 & & \\
\hline & ghuv74760_0492.rdb & 04-17-92 @ 1500 to 04-18-92 @ 1500 & 30 -minute & 15.83 & & \\
\hline & ghuv74760_0592.rdb & 05-27-92@0700 to 05-28-92 @0200 & 30-minute & 14.04 & & \\
\hline & ghuv74760_1192.rdb & 11-19-93@1800 to 11-20-93@1400 & 30-minute & 11.44 & & \\
\hline & ghuv74760_0393a.rdb & 03-01-93@0700 to 03-02-93 @ 1500 & 30 -minute & 14.41 & & \\
\hline & ghuv74760_0393b.rdb & 03-22-93@1900 to 03-23-93 @ 1100 & 30-minute & 11.75 & & \\
\hline & ghuv74760_0493.rdb & 04-03-93@2000 to 04-08-93 @ 0500 & 30-minute & 11.45 & & \\
\hline & ghuv74760_0693.rdb & 06-19-93@1400 to 06-21-93 @0600 & 30 -minute & 12.88 & & \\
\hline & ghuv74760_1093.rdb & 10-20-93@1300 to 10-21-93@ 1100 & 30 -minute & 11.28 & & \\
\hline
\end{tabular}


Appendix 2. Description of surface-water data-Continued

\begin{tabular}{|c|c|c|c|c|c|c|}
\hline $\begin{array}{c}\text { USGS } \\
\text { station no. }\end{array}$ & File name & $\begin{array}{l}\text { Period of unit-value data } \\
\text { (gage height and } \\
\text { (or) discharge }\end{array}$ & $\begin{array}{c}\text { Fre- } \\
\text { quency }\end{array}$ & $\begin{array}{c}\text { Maximum } \\
\text { gage height } \\
\text { (feet above } \\
\text { datum) }\end{array}$ & $\begin{array}{c}\text { Maximum } \\
\text { discharge } \\
\text { (cubic feet } / \\
\text { second) }\end{array}$ & Comments \\
\hline & ghuv74760_1193.rdb & 11-16-93@0800 to 11-17-93@0800 & 30-minute & 11.99 & & \\
\hline & ghuv74760_0294.rdb & 02-10-94@0800 to 02-10-94@2300 & 30-minute & 10.52 & & \\
\hline & ghuv74760_0994.rdb & 09-09-94@0600 to 09-09-94 @ 2300 & 30-minute & 12.30 & & \\
\hline & ghuv74760_1094.rdb & 10-15-94@1300 to 10-19-94@ @ 1400 & 30-minute & 16.83 & & \\
\hline & ghuv74760_0195a.rdb & 01-12-95@1900 to 01-13-95 @ 1300 & 30-minute & 14.23 & & \\
\hline & ghuv74760_0195b.rdb & 01-26-95 @ 1500 to 01-27-95 @ 1100 & 30-minute & 13.43 & & \\
\hline & ghuv74760_0495.rdb & 04-04-95@0800 to 04-06-95@0700 & 30-minute & 14.66 & & \\
\hline \multirow[t]{19}{*}{08074780} & ghuv74780_0191.rdb & 01-14-91@ @2000 to 01-16-91@0300 & 15 -minute & 76.15 & & \\
\hline & ghuv74780_0291.rdb & 02-04-91@0900 to 02-05-91 @ 1900 & 15-minute & 76.71 & & \\
\hline & ghuv74780_0491.rdb & 04-04-91@0800 to 04-16-91@0700 & 15-minute & 76.46 & & \\
\hline & ghuv74780_0292.rdb & 02-22-92@0400 to 02-23-92 @ 1900 & 15-minute & 76.64 & & \\
\hline & ghuv74780_0392.rdb & 03-04-92@0900 to 03-06-92 @ 1900 & 15 -minute & 80.93 & & \\
\hline & ghuv74780_0492.rdb & 04-17-92 @ 1400 to 04-21-92 @ 0200 & 15-minute & 78.65 & & \\
\hline & ghuv74780_0592.rdb & 05-28-92@ @ 1800 to 05-30-92 @ 0500 & 15-minute & 77.24 & & \\
\hline & ghuv74780_1192.rdb & 11-19-92@1800 to 11-22-92 @ 0700 & 15 -minute & 74.70 & & \\
\hline & ghuv74780_0393.rdb & 03-01-93@0800 to 03-03-93@ 0800 & 15-minute & 78.11 & & \\
\hline & ghuv74780_0493.rdb & 04-03-93@ @ 1700 to 04-05-93@ @ 0800 & 15-minute & 75.24 & & \\
\hline & ghuv74780_0693.rdb & 06-18-93@1000 to 06-22-93@0800 & 15-minute & 77.26 & & \\
\hline & ghuv74780_1093.rdb & $10-20-93 @ 1100$ to $10-21-93 @ 1600$ & 15 -minute & 75.84 & & \\
\hline & ghuv74780_1193.rdb & $11-16-93 @ 0600$ to $11-17-93 @ 1100$ & 15 -minute & 76.14 & & \\
\hline & ghuv74780_0694.rdb & 06-10-94@ @ 1100 to 06-11-94 @ 0100 & 15-minute & 75.09 & & \\
\hline & ghuv74780_0994.rdb & 09-09-94@0700 to 09-10-94@0100 & 15 -minute & 75.75 & & \\
\hline & ghuv74780_1094.rdb & 10-15-94@1300 to 10-20-94@ 1100 & 15-minute & 80.27 & & \\
\hline & ghuv74780_0195a.rdb & 01-12-95 @ 2000 to 01-13-95 @ 1900 & 15-minute & 77.34 & & \\
\hline & ghuv74780_0195b.rdb & 01-26-95 @ 1400 to 01-27-95 @ 1700 & 15-minute & 75.70 & & \\
\hline & ghuv74780_0495.rdb & 04-04-95 @ 0700 to 04-06-95 @ 1800 & 15-minute & 76.88 & & \\
\hline \multirow[t]{15}{*}{08074800} & quv74800_0191.rdb & 01-14-91@ @ 1300 to 01-17-91 @ 1900 & 15-minute & & 2,500 & \\
\hline & quv74800_0291.rdb & 02-04-91@0700 to 02-08-91@ @ 0800 & 15 -minute & & 2,410 & \\
\hline & quv74800_0491.rdb & 04-14-91@0800 to 04-17-91 @ 1100 & 15 -minute & & 1,890 & \\
\hline & quv74800_0691.rdb & 06-16-91@1800 to 06-18-91 @ 1800 & 15-minute & & 1,680 & \\
\hline & quv74800_0292.rdb & 02-22-92@0400 to 02-27-92@ @ 0900 & 15 -minute & & 2,480 & \\
\hline & quv74800_0392.rdb & 03-04-92@0100 to 03-09-92 @ 0700 & 15-minute & & 4,880 & \\
\hline & quv74800_0492.rdb & 04-17-92@1300 to 04-22-92 @ 0800 & 15-minute & & 3,400 & \\
\hline & quv74800_0592.rdb & 05-27-92@0500 to 05-31-92 @ 1100 & 15 -minute & & 2,420 & \\
\hline & quv74800_1192.rdb & 11-19-92@ @ 1400 to 11-23-92 @ 2200 & 15-minute & & 1,160 & \\
\hline & quv74800_0293.rdb & 02-25-93@0900 to 03-06-93@ @ 0800 & 15-minute & & 3,000 & \\
\hline & quv74800_0693.rdb & 06-17-93 @ 1900 to 06-23-93 @ 1100 & 15 -minute & & 1,670 & \\
\hline & quv74800_1093.rdb & 10-20-93@1100 to 10-23-93 @ 1000 & 15 -minute & & 2,040 & \\
\hline & quv74800_1193.rdb & 11-16-93@0400 to 11-19-93 @ 1900 & 15-minute & & 2,410 & \\
\hline & quv74800_0694.rdb & $06-10-94 @ 1500$ to $06-12-94 @ 0700$ & 15 -minute & & 1,080 & \\
\hline & quv74800_0994a.rdb & 09-03-94@1600 to 09-05-94 @ 0800 & 15-minute & & 1,620 & \\
\hline
\end{tabular}


Appendix 2. Description of surface-water data-Continued

\begin{tabular}{|c|c|c|c|c|c|c|}
\hline $\begin{array}{c}\text { USGS } \\
\text { station no. }\end{array}$ & File name & $\begin{array}{l}\text { Period of unit-value data } \\
\text { (gage height and } \\
\text { (or) discharge }\end{array}$ & $\begin{array}{c}\text { Fre- } \\
\text { quency }\end{array}$ & $\begin{array}{c}\text { Maximum } \\
\text { gage height } \\
\text { (feet above } \\
\text { datum) }\end{array}$ & $\begin{array}{c}\text { Maximum } \\
\text { discharge } \\
\text { (cubic feet/ } \\
\text { second) }\end{array}$ & Comments \\
\hline & quv74800_0994b.rdb & 09-09-94@0600 to 09-12-94@0700 & 15-minute & & 1,510 & \\
\hline & quv74800_1094.rdb & 10-15-94@0900 to 10-23-94@0800 & 15-minute & & 4,850 & \\
\hline & quv74800_0195a.rdb & 01-12-95 @ 2000 to 01-17-95 @ 0700 & 15-minute & & 2,810 & \\
\hline & quv74800_0195b.rdb & 01-25-95@2100 to 01-31-95@0700 & 15-minute & & 1,680 & \\
\hline & quv74800_0495.rdb & 04-04-95@0400 to 04-10-95@0800 & 15-minute & & 2,030 & \\
\hline \multirow[t]{23}{*}{08074810} & quv74810_0191a.rdb & 01-09-91@0800 to 01-13-91@ @ 1000 & 15-minute & & 6,570 & \\
\hline & quv74810_0191b.rdb & 01-14-91@ @ 1000 to 01-17-91@0900 & 15-minute & & 9,470 & \\
\hline & quv74810_0291.rdb & 02-04-91@0700 to 02-08-91@0700 & 15-minute & & 6,620 & \\
\hline & quv74810_0491a.rdb & 04-04-91@0700 to 04-09-91@ @ 1000 & 15-minute & & 6,560 & \\
\hline & quv74810_0491b.rdb & 04-14-91@0800 to 04-19-91@ @ 1000 & 15-minute & & 5,900 & \\
\hline & quv74810_0292.rdb & 02-22-92@0400 to 02-27-92 @ 0800 & 15 -minute & & 8,600 & \\
\hline & quv74810_0392.rdb & 03-04-92@0100 to 03-08-92 @ 1200 & 15 -minute & & 16,900 & $\begin{array}{l}\text { No data 03-04@ } 2015 \text { to 03-05 } \\
@ 1400\end{array}$ \\
\hline & quv74810_0492.rdb & 04-17-92@ @1100 to 04-23-92 @ 0800 & 15-minute & & 10,300 & \\
\hline & quv74810_0592.rdb & 05-27-92@0500 to 05-31-92 @ 0900 & 15-minute & & 5,960 & \\
\hline & quv74810_1192.rdb & 11-19-92@0900 to 11-24-92@ 1000 & 15-minute & & 3,400 & \\
\hline & quv74810_0293.rdb & 02-25-93@0800 to 03-06-93@0800 & 15-minute & & 8,380 & \\
\hline & quv74810_0393.rdb & 03-22-93@1600 to 03-26-93 @ 0900 & 15-minute & & 4,260 & \\
\hline & quv74810_0493.rdb & 04-03-93@1300 to 04-10-93@0900 & 15-minute & & 5,270 & \\
\hline & quv74810_0693.rdb & $06-17-93 @ 2100$ to 06-25-93 @ 1000 & 15-minute & & 7,650 & \\
\hline & quv74810_1093.rdb & $10-20-93 @ 0700$ to $10-23-93 @ 1000$ & 15-minute & & 5,630 & \\
\hline & quv74810_1193.rdb & $11-16-93 @ 0300$ to $11-19-93 @ 0900$ & 15-minute & & 7,900 & \\
\hline & quv74810_0694a.rdb & 06-10-94@1400 to 06-14-94@ @ 1000 & 15-minute & & 2,890 & \\
\hline & quv74810_0694b.rdb & 06-17-94@1700 to 06-26-94@ @ 1200 & 15-minute & & 2,920 & \\
\hline & quv74810_0994.rdb & 09-08-94@1300 to 09-12-94@ @ 1200 & 15-minute & & 4,100 & \\
\hline & quv74810_0195a.rdb & 01-12-95@0800 to 01-17-95@0800 & 15-minute & & 7,220 & \\
\hline & quv74810_0195b.rdb & 01-25-95@1800 to 01-30-95@0900 & 15-minute & & 5,590 & \\
\hline & quv74810_0395.rdb & 03-12-95@1100 to 03-17-95 @ 1300 & 15-minute & & 5,690 & \\
\hline & quv74810_0495.rdb & 04-03-95 @ 1500 to 04-09-95 @ 1200 & 15-minute & & 6,620 & \\
\hline \multirow[t]{13}{*}{08075000} & quv75000_0191.rdb & 01-14-91@0800 to 01-22-91@0800 & 30-minute & & 19,800 & \\
\hline & quv75000_0291.rdb & 02-04-91@0800 to 02-08-91@0900 & 30-minute & & 13,200 & \\
\hline & quv75000_0491a.rdb & 04-04-91@0200 to 04-10-91@0800 & 30-minute & & 13,400 & \\
\hline & quv75000_0491b.rdb & 04-14-91@0800 to 04-21-91@ @ 1000 & 30-minute & & 11,000 & \\
\hline & quv75000_0292.rdb & 02-22-92@0400 to 02-27-92 @ 0800 & 30-minute & & 18,100 & \\
\hline & quv75000_0392.rdb & 03-03-92@0700 to 03-11-92@0700 & 30-minute & & 23,000 & \\
\hline & quv75000_0492.rdb & 04-17-92@ @ 1500 to 04-22-92@0800 & 30-minute & & 17,400 & \\
\hline & quv75000_0592.rdb & 05-26-92@1900 to 05-31-92 @ 1000 & 30-minute & & 8,740 & \\
\hline & quv75000_1192.rdb & 11-19-92@0800 to 11-24-92@0800 & 30-minute & & 7,920 & \\
\hline & quv75000_0293.rdb & 02-28-93@0300 to 03-06-93@0800 & 30-minute & & 12,800 & \\
\hline & quv75000_0393.rdb & 03-22-93@0800 to 03-26-93@0700 & 30-minute & & 8,450 & \\
\hline & quv75000_0693.rdb & 06-17-93@0700 to 06-25-93@0800 & 30-minute & & 16,000 & \\
\hline & quv75000_1093.rdb & $10-20-93 @ 0800$ to $10-23-93 @ 0800$ & 30-minute & & 13,800 & \\
\hline
\end{tabular}


Appendix 2. Description of surface-water data-Continued

\begin{tabular}{|c|c|c|c|c|c|c|}
\hline $\begin{array}{c}\text { USGS } \\
\text { station no. }\end{array}$ & File name & $\begin{array}{l}\text { Period of unit-value data } \\
\text { (gage height and } \\
\text { (or) discharge }\end{array}$ & $\begin{array}{c}\text { Fre- } \\
\text { quency }\end{array}$ & $\begin{array}{l}\text { Maximum } \\
\text { gage height } \\
\text { (feet above } \\
\text { datum) }\end{array}$ & $\begin{array}{c}\text { Maximum } \\
\text { discharge } \\
\text { (cubic feet/ } \\
\text { second) }\end{array}$ & Comments \\
\hline & quv75000_1193.rdb & 11-13-93@1000 to 11-17-93@ 1600 & 15-minute & & 16,500 & No data after 11-17@1600 \\
\hline & quv75000_0594.rdb & 05-13-94@0800 to 05-20-94@0800 & 30-minute & & 5,840 & \\
\hline & quv75000_0694.rdb & 06-12-94@0800 to 06-27-94@0800 & 30-minute & & 7,540 & \\
\hline & quv75000_0994.rdb & 09-08-94@0800 to 09-12-94@0800 & 30-minute & & 4,990 & \\
\hline & quv75000_1094.rdb & 10-14-94@0800 to 10-23-94@ 1100 & 30-minute & & 27,000 & \\
\hline & quv75000_0195a.rdb & 01-12-95@0700 to 01-17-95@0900 & 30-minute & & 10,600 & \\
\hline & quv75000_0195b.rdb & 01-25-95@1900 to 01-30-95 @ 0900 & 30-minute & & 9,840 & \\
\hline & quv75000_0395.rdb & 03-12-95 @ 1000 to 03-18-95 @ 0900 & 30-minute & & 11,700 & \\
\hline & quv75000_0495.rdb & 04-03-95@0800 to 04-09-95 @ 1200 & 30-minute & & 12,100 & \\
\hline & quv75000_0595.rdb & 05-27-95@0700 to 06-04-95 @ 1000 & 30-minute & & 12,600 & \\
\hline \multirow[t]{17}{*}{08075400} & quv75400_0191a.rdb & 01-09-91@ @ 1200 to 01-12-91 @ 1000 & 15-minute & & 3,730 & \\
\hline & quv75400_0191b.rdb & 01-14-91@ @1900 to 01-17-91 @ 0700 & 15 -minute & & 5,120 & \\
\hline & quv75400_0291.rdb & 02-04-91@0700 to 02-07-91@ 1600 & 15-minute & & 2,390 & \\
\hline & quv75400_0491.rdb & 04-04-91 @ 1000 to 04-08-91 @ 2000 & 15-minute & & 4,080 & \\
\hline & quv75400_0292.rdb & 02-22-92@0300 to 02-27-92 @ 1400 & 15-minute & & 6,290 & \\
\hline & quv75400_0492.rdb & 04-17-92@1400 to 04-22-92 @ 0900 & 15 -minute & & 3,020 & \\
\hline & quv75400_0592.rdb & 05-28-92@ @ 1600 to 05-30-92 @ 2000 & 15 -minute & & 4,120 & \\
\hline & quv75400_0193.rdb & 01-07-93@0300 to 01-12-93@0900 & 15-minute & & 2,880 & \\
\hline & quv75400_0293.rdb & 02-28-93 @2100 to 03-05-93 @ 1000 & 15 -minute & & 2,710 & \\
\hline & quv75400_0493.rdb & 04-03-93@ @ 1500 to 04-11-93@ 1200 & 15-minute & & 2,440 & \\
\hline & quv75400_0693.rdb & 06-18-93@0400 to 06-24-93@ 1900 & 60-minute & & 4,540 & \\
\hline & quv75400_1093.rdb & $10-20-93 @ 1000$ to $10-22-93 @ 1600$ & 30-minute & & 3,710 & \\
\hline & quv75400_0694.rdb & 06-20-94 @ 1200 to 06-27-94 @ 1200 & 15-minute & & 2,150 & \\
\hline & quv75400_1094.rdb & 10-15-94@1000 to 10-23-94@ 1000 & 15-minute & & 7,510 & \\
\hline & quv75400_0395.rdb & 03-13-95@0600 to 03-18-95 @ 1600 & 15 -minute & & 2,130 & \\
\hline & quv75400_0495.rdb & 04-04-95 @0600 to 04-09-95 @ 1200 & 15-minute & & 2,680 & \\
\hline & quv75400_0595.rdb & 05-29-95@0700 to 06-04-95 @ 1000 & 15-minute & & 1,780 & \\
\hline \multirow[t]{14}{*}{08075500} & quv75500_0191.rdb & 01-09-91@ @1300 to 01-17-91 @ 1500 & 30-minute & & 7,040 & \\
\hline & quv75500_0291.rdb & 02-04-91@0600 to 02-09-91 @ 2300 & 30-minute & & 3,920 & \\
\hline & quv75500_0491.rdb & 04-04-91@0900 to 04-09-91 @ 1800 & 30-minute & & 6,250 & \\
\hline & quv75500_0192.rdb & 01-17-92@1200 to 01-21-92 @ 1700 & 30-minute & & 4,230 & \\
\hline & quv75500_0292a.rdb & 02-02-92 @2100 to 02-10-92 @ 1500 & 30-minute & & 4,490 & \\
\hline & quv75500_0292b.rdb & 02-22-92@0400 to 02-28-92 @ 1500 & 30-minute & & 4,410 & \\
\hline & quv75500_0592.rdb & 05-26-92@1900 to 06-05-92 @ 1900 & 30-minute & & 3,830 & \\
\hline & quv75500_0193.rdb & 01-06-93@1800 to 01-14-93 @ 1700 & 30-minute & & 2,810 & \\
\hline & quv75500_0293.rdb & 02-28-93@1800 to 03-05-93 @ 2000 & 30-minute & & 3,620 & \\
\hline & quv75500_0493.rdb & 04-03-93@1200 to 04-12-93 @ 0500 & 30-minute & & 3,230 & \\
\hline & quv75500_0693.rdb & 06-17-93@1300 to 06-25-93 @ 1700 & 30-minute & & 4,180 & \\
\hline & quv75500_1093.rdb & 10-20-93@1000 to 10-23-93@2000 & 30-minute & & 4,770 & \\
\hline & quv75500_1193.rdb & $11-14-93 @ 0800$ to 11-20-93 @ 2100 & 30-minute & & 4,520 & \\
\hline & quv75500_0594.rdb & 05-05-94@0600 to 05-19-94@0900 & 30-minute & & 2,290 & \\
\hline
\end{tabular}


Appendix 2. Description of surface-water data-Continued

\begin{tabular}{|c|c|c|c|c|c|c|}
\hline $\begin{array}{c}\text { USGS } \\
\text { station no. }\end{array}$ & File name & $\begin{array}{l}\text { Period of unit-value data } \\
\text { (gage height and } \\
\text { (or) discharge }\end{array}$ & $\begin{array}{c}\text { Fre- } \\
\text { quency }\end{array}$ & $\begin{array}{l}\text { Maximum } \\
\text { gage height } \\
\text { (feet above } \\
\text { datum) }\end{array}$ & $\begin{array}{c}\text { Maximum } \\
\text { discharge } \\
\text { (cubic feet/ } \\
\text { second) }\end{array}$ & Comments \\
\hline & quv75500_0694.rdb & 06-20-94@1200 to 06-27-94 @ 2300 & 30-minute & & 1,910 & \\
\hline & quv75500_1094.rdb & 10-15-94@0300 to 10-23-94@1800 & 30-minute & & 7,730 & \\
\hline & quv75500_0395.rdb & 03-12-95@1800 to 03-18-95@ @ 1800 & 30-minute & & 2,370 & \\
\hline & quv75500_0495.rdb & 04-04-95@0100 to 04-09-95 @1800 & 30-minute & & 2,780 & \\
\hline \multirow[t]{12}{*}{08075650} & ghuv75650_0492.rdb & 04-05-92@0500 to 04-08-92@0700 & 30-minute & 11.04 & & \\
\hline & ghuv75650_0592.rdb & 05-27-92@0400 to 05-28-92 @ 0100 & 30-minute & 11.31 & & \\
\hline & ghuv75650_0692.rdb & 06-02-92@0500 to 06-03-92@0800 & 30-minute & 14.17 & & \\
\hline & ghuv75650_0293.rdb & 02-28-93@0900 to 03-03-93@1400 & 30-minute & 12.07 & & \\
\hline & ghuv75650_0393.rdb & 03-21-93@1700 to 03-24-93@1000 & 30-minute & 8.99 & & \\
\hline & ghuv75650_0493.rdb & 04-02-93@1000 to 04-10-93@0700 & 30-minute & 12.07 & & \\
\hline & ghuv75650_1093.rdb & 10-19-93@2000 to 10-22-93@0400 & 30-minute & 15.61 & & \\
\hline & ghuv75650_1193.rdb & 11-13-93@1600 to 11-17-93@2100 & 30-minute & 13.27 & & \\
\hline & ghuv75650_0194.rdb & 01-26-94@1300 to 01-28-94@2100 & 30-minute & 11.79 & & \\
\hline & ghuv75650_1094.rdb & 10-13-94@0100 to 10-21-94@1600 & 30-minute & 17.97 & & \\
\hline & ghuv75650_0495.rdb & 04-03-95@0600 to 04-06-95@0800 & 30-minute & 12.91 & & \\
\hline & ghuv75650_0595.rdb & 05-29-95@0400 to 06-02-95@0800 & 30-minute & 13.87 & & \\
\hline \multirow[t]{19}{*}{08075730} & quv75730_1089.rdb & 10-29-89@1400 to 11-01-89@0100 & 15-minute & & 2,170 & \\
\hline & quv75730_0490.rdb & 04-26-90@1400 to 04-28-90 @2300 & 15-minute & & 678 & \\
\hline & quv75730_0890.rdb & 08-15-90@1600 to 08-16-90 @ 1400 & 15-minute & & 504 & \\
\hline & quv75730_0191.rdb & 01-14-91@1800 to 01-17-91@2300 & 15-minute & & 1,820 & \\
\hline & quv75730_0291.rdb & 02-04-91@0700 to 02-07-91@2300 & 15-minute & & 1,750 & \\
\hline & quv75730_0491.rdb & 04-04-91@ @ 1000 to 04-08-91@ @ 1500 & 15-minute & & 2,060 & \\
\hline & quv75730_0591.rdb & 05-29-91@1000 to 05-31-91@2300 & 15-minute & & 2,210 & \\
\hline & quv75730_1291.rdb & 12-21-91@0900 to 12-24-91@0500 & 15-minute & & 1,430 & \\
\hline & quv75730_0492.rdb & 04-19-92@2000 to 04-21-92 @2300 & 15-minute & & 1,280 & \\
\hline & quv75730_0592.rdb & 05-26-92@2100 to 05-30-92 @ 1900 & 15-minute & & 1,420 & \\
\hline & quv75730_0692.rdb & 06-01-92@1400 to 06-05-92 @ 1800 & 15-minute & & 1,830 & \\
\hline & quv75730_0293.rdb & 02-28-93@2100 to 03-04-93@1600 & 15-minute & & 1,750 & \\
\hline & quv75730_0793.rdb & 07-19-93@1100 to 07-20-93@2100 & 15-minute & & 1,610 & \\
\hline & quv75730_1093.rdb & 10-20-93@1100 to 10-24-93@1300 & 30-minute & & 2,030 & \\
\hline & quv75730_1193.rdb & 11-14-93@0700 to 11-18-93@1200 & 15-minute & & 1,510 & \\
\hline & quv75730_0194.rdb & 01-27-94@0600 to 01-31-94@0800 & 15-minute & & 1,600 & \\
\hline & quv75730_1094.rdb & 10-15-94@0545 to 10-21-94@0915 & 30-minute & & 2,710 & \\
\hline & quv75730_0495.rdb & 04-03-95@1800 to 04-07-95@1700 & 15-minute & & 1,660 & \\
\hline & quv75730_0595.rdb & 05-29-95@0400 to 06-03-95@0200 & 15-minute & & 2,000 & \\
\hline \multirow[t]{6}{*}{08075770} & quv75770_0390.rdb & 03-28-90@0100 to 04-05-90 @ 1000 & 30-minute & & 594 & \\
\hline & quv75770_0490.rdb & 04-26-90@1200 to 05-01-90@1800 & 30-minute & & 850 & \\
\hline & quv75770_0790.rdb & 07-05-90@0800 to 07-08-90 @ 1700 & 30-minute & & 604 & \\
\hline & quv75770_0191a.rdb & 01-09-91@0700 to 01-13-91@1000 & 30-minute & & 1,530 & \\
\hline & quv75770_0191b.rdb & 01-14-91@1600 to 01-21-91@ @ 1300 & 30-minute & & 1,900 & \\
\hline & quv75770_0491a.rdb & 04-04-91@0900 to 04-09-91@0700 & 30-minute & & 1,630 & \\
\hline
\end{tabular}


Appendix 2. Description of surface-water data-Continued

\begin{tabular}{|c|c|c|c|c|c|c|}
\hline $\begin{array}{c}\text { USGS } \\
\text { station no. }\end{array}$ & File name & $\begin{array}{l}\text { Period of unit-value data } \\
\text { (gage height and } \\
\text { (or) discharge }\end{array}$ & $\begin{array}{c}\text { Fre- } \\
\text { quency }\end{array}$ & $\begin{array}{c}\text { Maximum } \\
\text { gage height } \\
\text { (feet above } \\
\text { datum) }\end{array}$ & $\begin{array}{c}\text { Maximum } \\
\text { discharge } \\
\text { (cubic feet/ } \\
\text { second) }\end{array}$ & Comments \\
\hline & quv75770_0491b.rdb & 04-14-91@0800 to 04-19-91 @ 2300 & 30-minute & & 1,290 & \\
\hline & quv75770_1291.rdb & $12-21-91 @ 0800$ to 12-25-91@0400 & 30-minute & & 1,060 & \\
\hline & quv75770_0392.rdb & 03-04-92@0100 to 03-09-92 @ 2100 & 30-minute & & 2,800 & \\
\hline & quv75770_0592.rdb & 05-27-92@0600 to 05-30-92 @ 2300 & 30-minute & & 742 & \\
\hline & quv75770_0692.rdb & 06-01-92@ @ 1400 to 06-05-92 @ 1400 & 30-minute & & 1,120 & \\
\hline & quv75770_0293.rdb & 02-28-93@2100 to 03-05-93 @ 2300 & 30-minute & & 1,810 & \\
\hline & quv75770_0393.rdb & 03-22-93@0400 to 03-27-93@ 2300 & 30-minute & & 2,890 & \\
\hline & quv75770_1093.rdb & 10-20-93@0900 to 10-25-93@0900 & 30-minute & & 1,910 & \\
\hline & quv75770_1193.rdb & 11-14-93@0200 to 11-21-93@0500 & 30-minute & & 1,970 & \\
\hline & quv75770_0594.rdb & 05-13-94@0800 to 05-21-94@0900 & 30-minute & & 1,630 & \\
\hline & quv75770_1094.rdb & 10-15-94@0800 to 10-24-94@ 1100 & 30-minute & & 3,430 & \\
\hline & quv75770_0195.rdb & 01-26-95@0000 to 01-30-95 @ 2300 & 30-minute & & 1,560 & \\
\hline & quv75770_0495.rdb & 04-04-95@0600 to 04-10-95 @ 0800 & 30-minute & & 1,170 & \\
\hline & quv75770_0595.rdb & 05-29-95 @0300 to 06-04-95 @ 1500 & 30-minute & & 713 & \\
\hline \multirow[t]{20}{*}{08075780} & quv75780_0290.rdb & 02-21-90@0900 to 02-23-90@0100 & 30-minute & & 552 & \\
\hline & quv75780_0390.rdb & 03-28-90@ @ 1000 to 03-31-90 @ 1700 & 30-minute & & 252 & \\
\hline & quv75780_0490.rdb & 04-26-90 @ 1300 to 04-29-90 @ 1300 & 30-minute & & 264 & \\
\hline & quv75780_0191.rdb & 01-18-91@0700 to 01-20-91@0600 & 30-minute & & 424 & \\
\hline & quv75780_0491a.rdb & 04-04-91@ @ 1000 to 04-07-91@ @2300 & 30-minute & & 510 & \\
\hline & quv75780_0491b.rdb & 04-14-91@0800 to 04-16-91@ @2000 & 30-minute & & 889 & \\
\hline & quv75780_1191.rdb & $11-17-91 @ 0600$ to 11-19-91@ 1000 & 30-minute & & 904 & \\
\hline & quv75780_0392.rdb & 03-04-92@ 1000 to 03-06-92 @ 0100 & 30-minute & & 557 & \\
\hline & quv75780_0492.rdb & 04-17-92@1300 to 04-19-92 @ 1300 & 30-minute & & 508 & \\
\hline & quv75780_0692.rdb & 06-01-92@ @ 1500 to 06-04-92@0700 & 30-minute & & 697 & \\
\hline & quv75780_0393a.rdb & 03-01-93@0800 to 03-04-93 @ 0200 & 30 -minute & & 715 & \\
\hline & quv75780_0393b.rdb & 03-22-93@1500 to 03-24-93 @ 1800 & 30-minute & & 542 & \\
\hline & quv75780_0493.rdb & 04-03-93 @ 1100 to 04-09-93 @ 1800 & 30-minute & & 538 & \\
\hline & quv75780_0693.rdb & 06-17-93@1800 to 06-23-93 @ 1200 & 30-minute & & 516 & \\
\hline & quv75780_0194.rdb & 01-27-94@1000 to 01-28-94@0500 & 30-minute & & 378 & \\
\hline & quv75780_0294.rdb & 02-09-94 @ 1900 to 02-11-94 @2100 & 30-minute & & 640 & \\
\hline & quv75780_0594.rdb & 05-13-94@ @ 1700 to 05-17-94@0700 & 30-minute & & 533 & \\
\hline & quv75780_0195.rdb & 01-26-95 @ 1600 to 01-28-95@0300 & 30-minute & & 221 & \\
\hline & quv75780_0495.rdb & 04-04-95 @0600 to 04-06-95 @0400 & 30-minute & & 428 & \\
\hline & quv75780_0595.rdb & 05-30-95@0600 to 05-30-95 @ 2330 & 30-minute & & 157 & \\
\hline \multirow[t]{7}{*}{08075900} & quv75900_0290.rdb & $02-21-90 @ 0600$ to 02-24-90 @ 1400 & 30-minute & & 2,930 & \\
\hline & quv75900_0390.rdb & 03-28-90@0900 to 04-02-90 @ 1500 & 30-minute & & 1,600 & \\
\hline & quv75900_0490.rdb & 04-26-90 @ 1200 to 05-01-90 @ 1600 & 30-minute & & 1,770 & \\
\hline & quv75900_0191.rdb & 01-14-91@ @ 1300 to 01-22-91@ 1700 & 30-minute & & 2,630 & \\
\hline & quv75900_0291.rdb & 02-04-91@0700 to 02-08-91@ 1700 & 30-minute & & 2,130 & \\
\hline & quv75900_0491.rdb & 04-04-91@0300 to 04-06-91@ 1500 & 30-minute & & 3,530 & $\begin{array}{l}\text { No record 04-06@1500 through } \\
\text { peak }\end{array}$ \\
\hline & quv75900_0392.rdb & 03-04-92@0100 to 03-08-92 @ 1100 & 30-minute & & 8,180 & \\
\hline
\end{tabular}


Appendix 2. Description of surface-water data-Continued

\begin{tabular}{|c|c|c|c|c|c|c|}
\hline $\begin{array}{c}\text { USGS } \\
\text { station no. }\end{array}$ & File name & $\begin{array}{l}\text { Period of unit-value data } \\
\text { (gage height and } \\
\text { (or) discharge }\end{array}$ & $\begin{array}{c}\text { Fre- } \\
\text { quency }\end{array}$ & \begin{tabular}{|c} 
Maximum \\
gage height \\
(feet above \\
datum)
\end{tabular} & $\begin{array}{l}\text { Maximum } \\
\text { discharge } \\
\text { (cubic feet/ } \\
\text { second) }\end{array}$ & Comments \\
\hline & quv75900_0592.rdb & 05-16-92@0600 to 05-21-92@0800 & 30-minute & & 5,570 & \\
\hline & quv75900_0692.rdb & 06-01-92@1300 to 06-05-92 @2000 & 30-minute & & 2,840 & \\
\hline & quv75900_0393a.rdb & 03-01-93@0600 to 03-05-93@ @2300 & 30-minute & & 5,500 & \\
\hline & quv75900_0393b.rdb & 03-22-93@1500 to 03-27-93@0900 & 30-minute & & 2,440 & \\
\hline & quv75900_0493.rdb & 04-03-93@1000 to 04-12-93@0700 & 30-minute & & 2,890 & \\
\hline & quv75900_0593.rdb & 05-23-93@1100 to 05-30-93@0900 & 30-minute & & 3,020 & \\
\hline & quv75900_0693.rdb & 06-17-93@1600 to 06-30-93@1400 & 30-minute & & 1,370 & \\
\hline & quv75900_1093.rdb & 10-20-93@1100 to 10-23-93@0500 & 30-minute & & 1,400 & \\
\hline & quv75900_1193.rdb & 11-13-93@2000 to 11-20-93@0800 & 30-minute & & 1,560 & \\
\hline & quv75900_0294.rdb & 02-09-94@1200 to 02-15-94@0800 & 30-minute & & 1,940 & \\
\hline & quv75900_0594.rdb & 05-13-94@0800 to 05-20-94@0800 & 30-minute & & 1,230 & \\
\hline & quv75900_1094.rdb & 10-15-94@0300 to 10-22-94@1000 & 30-minute & & 6,150 & \\
\hline & quv75900_0195.rdb & 01-26-95@0200 to 01-30-95 @1500 & 30-minute & & 1,970 & \\
\hline & quv75900_0495.rdb & 04-04-95@0000 to 04-09-95@0300 & 30-minute & & 1,820 & \\
\hline \multirow[t]{22}{*}{08076000} & quv76000_0290.rdb & 02-21-90@0600 to 02-24-90 @ 1600 & 30-minute & & 2,190 & \\
\hline & quv76000_0390.rdb & 03-28-90@0100 to 04-05-90@1000 & 30-minute & & 2,040 & \\
\hline & quv76000_0490.rdb & 04-26-90@1000 to 05-02-90 @ 1300 & 30-minute & & 2,810 & \\
\hline & quv76000_0191a.rdb & 01-01-91@1500 to 01-13-91 @1500 & 30-minute & & 2,500 & \\
\hline & quv76000_0191b.rdb & 01-14-91@1500 to 01-22-91 @ 1200 & 30-minute & & 3,160 & \\
\hline & quv76000_0291.rdb & 02-04-91@0800 to 02-09-91@ @ 1000 & 30-minute & & 2,610 & \\
\hline & quv76000_0491.rdb & 04-04-91@0600 to 04-20-91 @1500 & 30-minute & & 4,280 & \\
\hline & quv76000_1191.rdb & 11-17-91@0500 to 11-21-91@ @ 1200 & 30-minute & & 4,700 & \\
\hline & quv76000_1291.rdb & 12-21-91@0400 to 12-25-91@ @ 1800 & 30-minute & & 5,120 & \\
\hline & quv76000_0392.rdb & 03-04-92@0100 to 03-09-92 @ 1400 & 30-minute & & 9,560 & \\
\hline & quv76000_0293.rdb & 02-25-93@0100 to 03-06-93@1900 & 30-minute & & 5,620 & \\
\hline & quv76000_0393.rdb & 03-22-93@1200 to 03-27-93@1500 & 30-minute & & 2,880 & \\
\hline & quv76000_0493.rdb & 04-03-93@1000 to 04-13-93@1200 & 30-minute & & 3,290 & \\
\hline & quv76000_0693.rdb & 06-17-93@1500 to 07-01-93@1000 & 30-minute & & 2,440 & \\
\hline & quv76000_1093.rdb & 10-19-93@1100 to 10-24-93@1000 & 30-minute & & 2,060 & \\
\hline & quv76000_1193.rdb & 11-13-93@1600 to 11-20-93@1600 & 30-minute & & 2,080 & \\
\hline & quv76000_0594.rdb & 05-13-94@1200 to 05-21-94@1600 & 30-minute & & 3,150 & \\
\hline & quv76000_0894.rdb & 08-15-94@2100 to 08-20-94@0900 & 30-minute & & 1,980 & \\
\hline & quv76000_1094.rdb & 10-15-94@0900 to 10-26-94@1300 & 30-minute & & 7,150 & \\
\hline & quv76000_0195.rdb & 01-25-95@1400 to 02-02-95@2200 & 30-minute & & 2,890 & \\
\hline & quv76000_0395.rdb & 03-12-95@1500 to 03-22-95@1400 & 30-minute & & 2,550 & \\
\hline & quv76000_0495.rdb & 04-04-95@0700 to 04-14-95@ @ 1500 & 30-minute & & 2,310 & \\
\hline \multirow[t]{5}{*}{08076180} & quv76180_0390.rdb & 03-28-90@0600 to 04-01-90@1100 & 60-minute & & 978 & \\
\hline & quv76180_0490.rdb & 04-26-90@1100 to 04-30-90 @ 0800 & 60-minute & & 1,540 & \\
\hline & quv76180_0590.rdb & 05-03-90@0900 to 05-06-90 @1900 & 60-minute & & 745 & \\
\hline & quv76180_0191.rdb & 01-09-91@0900 to 01-23-91@0700 & 60-minute & & 1,480 & \\
\hline & quv76180_0291.rdb & 02-04-91@0900 to 02-08-91@ @ 1300 & 60-minute & & 1,450 & \\
\hline
\end{tabular}


Appendix 2. Description of surface-water data-Continued

\begin{tabular}{|c|c|c|c|c|c|c|}
\hline $\begin{array}{c}\text { USGS } \\
\text { station no. }\end{array}$ & File name & $\begin{array}{l}\text { Period of unit-value data } \\
\text { (gage height and } \\
\text { (or) discharge }\end{array}$ & $\begin{array}{c}\text { Fre- } \\
\text { quency }\end{array}$ & $\begin{array}{l}\text { Maximum } \\
\text { gage height } \\
\text { (feet above } \\
\text { datum) }\end{array}$ & $\begin{array}{c}\text { Maximum } \\
\text { discharge } \\
\text { (cubic feet/ } \\
\text { second) }\end{array}$ & Comments \\
\hline & quv76180_0491.rdb & 04-04-91@ @1300 to 04-19-91@ 1400 & 60-minute & & 1,810 & \\
\hline & quv76180_0691.rdb & 06-13-91@ @ 1100 to 06-20-91@ 1800 & 60-minute & & 2,610 & \\
\hline & quv76180_1191.rdb & 11-17-91@0400 to 11-21-91@ 1100 & 60-minute & & 2,240 & \\
\hline & quv76180_1291.rdb & 12-21-91@0400 to 12-26-91@0700 & 60-minute & & 2,090 & \\
\hline & quv76180_0392.rdb & 03-04-92@0000 to 03-11-92 @ 1200 & 60-minute & & 9,980 & \\
\hline & quv76180_0592.rdb & 05-27-92@0800 to 06-07-92 @ 2000 & 60-minute & & 2,030 & \\
\hline & quv76180_0293.rdb & 02-25-93@0900 to 03-06-93 @ 1400 & 60-minute & & 2,930 & \\
\hline & quv76180_0393.rdb & 03-22-93@1400 to 03-28-93@0800 & 60-minute & & 2,080 & \\
\hline & quv76180_0493.rdb & 04-03-93@0500 to 04-13-93@0000 & 60-minute & & 2,010 & \\
\hline & quv76180_0693.rdb & 06-17-93@0600 to 07-01-93@ 1800 & 60-minute & & 3,900 & \\
\hline & quv76180_1093.rdb & 10-20-93@0900 to 10-23-93@ 1400 & 60-minute & & 833 & \\
\hline & quv76180_1193.rdb & 11-14-93@0800 to 11-21-93 @ 1600 & 60-minute & & 2,370 & \\
\hline & quv76180_0594.rdb & 05-13-94@0700 to 05-20-94@0700 & 60-minute & & 1,800 & \\
\hline & quv76180_0694.rdb & 06-01-94@2200 to 06-06-94 @ 1600 & 60-minute & & 945 & \\
\hline & quv76180_1094.rdb & 10-15-94@1200 to 10-26-94@ 1100 & 60-minute & & 3,940 & \\
\hline & quv76180_0195.rdb & 01-26-95@0300 to 01-31-95@2200 & 60-minute & & 1,690 & \\
\hline & quv76180_0395.rdb & 03-13-95@0100 to 03-20-95@0800 & 60-minute & & 1,370 & \\
\hline \multirow[t]{21}{*}{08076500} & quv76500_1089.rdb & 10-29-89@1200 to 11-02-89@ 1400 & 30-minute & & 730 & \\
\hline & quv76500_0390.rdb & 03-28-90@1000 to 04-05-90 @ 1300 & 30-minute & & 943 & \\
\hline & quv76500_0490.rdb & 04-26-90@1200 to 05-01-90 @ 0200 & 30-minute & & 1,120 & \\
\hline & quv76500_0191.rdb & 01-14-91@ @1400 to 01-21-91@ 1300 & 30-minute & & 1,250 & \\
\hline & quv76500_0491.rdb & 04-04-91@0600 to 04-20-91@ 1500 & 30-minute & & 1,890 & \\
\hline & quv76500_0591.rdb & 05-15-91@ @ 1400 to 05-18-91@ 1100 & 30-minute & & 1,530 & \\
\hline & quv76500_1191.rdb & 11-17-91@0600 to 11-20-91@ 2300 & 30-minute & & 2,260 & \\
\hline & quv76500_1291.rdb & 12-21-91@0400 to 12-25-91@ 1600 & 30-minute & & 2,150 & \\
\hline & quv76500_0392.rdb & 03-04-92@0100 to 03-08-92 @ 1300 & 30-minute & & 4,840 & \\
\hline & quv76500_0492.rdb & 04-17-92@1200 to 04-22-92 @ 1200 & 30-minute & & 2,580 & \\
\hline & quv76500_0293.rdb & 02-25-93@0800 to 03-06-93@1300 & 30-minute & & 2,560 & \\
\hline & quv76500_0393.rdb & 03-22-93@1300 to 03-28-93@ 1400 & 30-minute & & 2,650 & \\
\hline & quv76500_0493.rdb & 04-03-93 @ 1100 to 04-12-93 @ 1200 & 30-minute & & 1,940 & \\
\hline & quv76500_0693.rdb & 06-17-93@1000 to 07-01-93@ 1500 & 30-minute & & 794 & \\
\hline & quv76500_1093.rdb & 10-20-93@0800 to 10-23-93@ 1200 & 30-minute & & 602 & \\
\hline & quv76500_1193.rdb & $11-13-93 @ 1400$ to $11-19-93 @ 2200$ & 30-minute & & 1,580 & \\
\hline & quv76500_0594.rdb & 05-13-94@1100 to 05-20-94@ 1700 & 30-minute & & 1,940 & \\
\hline & quv76500_0894.rdb & 08-14-94@1800 to 08-20-94@ @ 1500 & 30-minute & & 717 & \\
\hline & quv76500_1094.rdb & 10-15-94@0300 to 10-23-94@ 1400 & 30-minute & & 2,580 & \\
\hline & quv76500_0195.rdb & 01-26-95@0300 to 01-31-95@0800 & 30-minute & & 1,820 & \\
\hline & quv76500_0395.rdb & 03-13-95@0300 to 03-18-95 @ 1700 & 30-minute & & 1,380 & \\
\hline \multirow[t]{3}{*}{08076700} & quv76700_0191.rdb & 01-09-91@0800 to 01-22-91@0900 & 60-minute & & 6,170 & \\
\hline & quv76700_0291.rdb & 02-04-91@0600 to 02-08-91@ 2300 & 60-minute & & 5,490 & \\
\hline & quv76700_0691.rdb & 06-15-91@0700 to 06-18-91@ 1900 & 60-minute & & 5,110 & \\
\hline
\end{tabular}


Appendix 2. Description of surface-water data-Continued

\begin{tabular}{|c|c|c|c|c|c|c|}
\hline $\begin{array}{c}\text { USGS } \\
\text { station no. }\end{array}$ & File name & $\begin{array}{l}\text { Period of unit-value data } \\
\text { (gage height and } \\
\text { (or) discharge }\end{array}$ & $\begin{array}{c}\text { Fre- } \\
\text { quency }\end{array}$ & $\begin{array}{c}\text { Maximum } \\
\text { gage height } \\
\text { (feet above } \\
\text { datum) }\end{array}$ & $\begin{array}{c}\text { Maximum } \\
\text { discharge } \\
\text { (cubic feet } / \\
\text { second) }\end{array}$ & Comments \\
\hline & quv76700_0293.rdb & 02-28-93@1600 to 03-04-93 @ 1500 & 60-minute & & 13,700 & \\
\hline & quv76700_0493.rdb & 04-07-93@0500 to 04-10-93@0000 & 60-minute & & 8,350 & \\
\hline & quv76700_0693.rdb & 06-17-93@0700 to 06-29-93 @ 2100 & 60-minute & & 7,280 & \\
\hline & quv76700_1093.rdb & 10-18-93@0100 to 10-22-93@0600 & 60-minute & & 2,380 & \\
\hline & quv76700_1193.rdb & 11-13-93@1900 to 11-18-93 @ 1000 & 60-minute & & 4,370 & \\
\hline & quv76700_0594.rdb & 05-13-94@1200 to 05-18-94@ 1000 & 60-minute & & 8,130 & \\
\hline & quv76700_0894.rdb & 08-17-94@1600 to 08-18-94 @ 2000 & 60-minute & & 8,840 & \\
\hline & quv76700_1094.rdb & 10-15-94@0000 to 10-22-94@0000 & \begin{tabular}{|l} 
See \\
comments
\end{tabular} & & 21,800 & Random data each day \\
\hline & quv76700_0195.rdb & 01-26-95 @ 1200 to 01-29-95 @ 1200 & 60-minute & & 8,690 & \\
\hline & quv76700_0395.rdb & 03-13-95@0300 to 03-17-95 @ 1800 & 60-minute & & 5,590 & \\
\hline & quv76700_0495.rdb & 04-04-95 @0700 to 04-12-95 @ 1400 & 60-minute & & 5,050 & \\
\hline
\end{tabular}


